\title{
The singular value decomposition
}

DOI:

10.1137/17M1117732

\section{Document Version}

Final published version

Link to publication record in Manchester Research Explorer

\section{Citation for published version (APA):}

Dongarra, J., Gates, M., Haidar, A., Kurzak, J., Luszczek, P., Tomov, S., \& Yamazaki, I. (2018). The singular value decomposition: Anatomy of optimizing an algorithm for extreme scale. SIAM REVIEW, 60(4), 808-865.

https://doi.org/10.1137/17M1117732

\section{Published in:}

SIAM REVIEW

\section{Citing this paper}

Please note that where the full-text provided on Manchester Research Explorer is the Author Accepted Manuscript or Proof version this may differ from the final Published version. If citing, it is advised that you check and use the publisher's definitive version.

\section{General rights}

Copyright and moral rights for the publications made accessible in the Research Explorer are retained by the authors and/or other copyright owners and it is a condition of accessing publications that users recognise and abide by the legal requirements associated with these rights.

\section{Takedown policy}

If you believe that this document breaches copyright please refer to the University of Manchester's Takedown Procedures [http://man.ac.uk/04Y6Bo] or contact uml.scholarlycommunications@manchester.ac.uk providing relevant details, so we can investigate your claim.

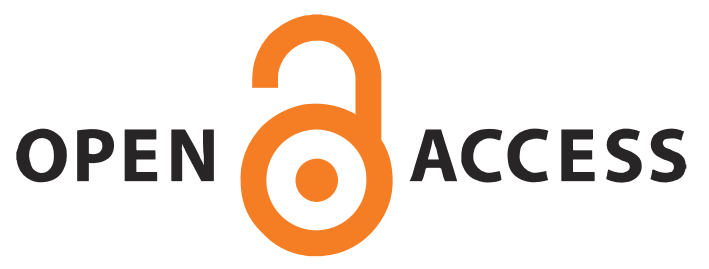




\title{
The Singular Value \\ Decomposition: Anatomy of Optimizing an Algorithm for Extreme Scale*
}

\author{
Jack Dongarra ${ }^{\dagger}$ \\ Mark Gates $\ddagger$ \\ Azzam Haidar $\ddagger$ \\ Jakub Kurzak $\ddagger$ \\ Piotr Luszczek ${ }^{\ddagger}$ \\ Stanimire Tomov \\ Ichitaro Yamazaki ${ }^{\ddagger}$
}

\begin{abstract}
The computation of the singular value decomposition, or SVD, has a long history with many improvements over the years, both in its implementations and algorithmically. Here, we survey the evolution of SVD algorithms for dense matrices, discussing the motivation and performance impacts of changes. There are two main branches of dense SVD methods: bidiagonalization and Jacobi. Bidiagonalization methods started with the implementation by Golub and Reinsch in Algol60, which was subsequently ported to Fortran in the EISPACK library, and was later more efficiently implemented in the LINPACK library, targeting contemporary vector machines. To address cache-based memory hierarchies, the SVD algorithm was reformulated to use Level 3 BLAS in the LAPACK library. To address new architectures, ScaLAPACK was introduced to take advantage of distributed computing, and MAGMA was developed for accelerators such as GPUs. Algorithmically, the divide and conquer and MRRR algorithms were developed to reduce the number of operations. Still, these methods remained memory bound, so two-stage algorithms were developed to reduce memory operations and increase the computational intensity, with efficient implementations in PLASMA, DPLASMA, and MAGMA. Jacobi methods started with the two-sided method of Kogbetliantz and the one-sided method of Hestenes. They have likewise had many developments, including parallel and block versions and preconditioning to improve convergence. In this paper, we investigate the impact of these changes by testing various historical and current implementations on a common, modern multicore machine and a distributed computing platform. We show that algorithmic and implementation improvements have increased the speed of the SVD by several orders of magnitude, while using up to 40 times less energy.
\end{abstract}

Key words. singular value decomposition, SVD, bidiagonal matrix, QR iteration, divide and conquer, bisection, MRRR, Jacobi method, Kogbetliantz method, Hestenes method

\footnotetext{
* Received by the editors February 21, 2017; accepted for publication (in revised form) March 22, 2018; published electronically November 8, 2018.

http://www.siam.org/journals/sirev/60-4/M111773.html

Funding: This research is based upon work supported by the National Science Foundation under grant 1339822 and by the Exascale Computing Project (17-SC-20-SC), a collaborative effort of the U.S. Department of Energy Office of Science and the National Nuclear Security Administration. This work also received support from NVIDIA and Intel.

$\dagger$ Innovative Computing Laboratory, University of Tennessee, Knoxville, TN 37996, Oak Ridge National Laboratory, University of Manchester (dongarra@icl.utk.edu).

${ }^{\ddagger}$ Innovative Computing Laboratory, University of Tennessee, Knoxville, TN 37996 (mgates3@ icl.utk.edu, haidar@icl.utk.edu, kurzak@icl.utk.edu, luszczek@icl.utk.edu, tomov@icl.utk.edu, iyamazak@icl.utk.edu).
} 
AMS subject classifications. 15A18, 15A23, 65Y05

DOI. $10.1137 / 17 \mathrm{M} 1117732$

\section{Contents}

Introduction

2 Experimental Setup $\quad 8$ II

$\begin{array}{llr}3 & \text { EISPACK Implementation } & 812\end{array}$

4 LINPACK Implementation Using BLAS $\quad 814$

5 LAPACK Implementation Based on Blocked Householder Transforma$\begin{array}{ll}\text { tions } & 814\end{array}$

5.1 Blocked Householder Transformations . . . . . . . . . . . . . . . 814

5.2 QR Iteration . . . . . . . . . . . . . . . . . . . . 817

5.3 Computation of Singular Vectors . . . . . . . . . . . . . . 818

5.4 Initial QR Factorization . . . . . . . . . . . . . . . . . 818

5.5 Results . . . . . . . . . . . . . . . . . . . . . . . . . . . 820

5.6 Level 2.5 BLAS Implementation . . . . . . . . . . . . . . 820

6 ScaLAPACK Implementation $\quad 822$

7 Singular Vectors from the Divide and Conquer Process 824

$\begin{array}{lll}8 & \text { Bisection and Inverse Iteration } & \mathbf{8 2 7}\end{array}$

9 Multiple Relatively Robust Representations (MRRR) 829

10 MAGMA Implementation for Accelerator Architectures 833

I I Two-Stage Reduction $\quad \mathbf{8 3 5}$

11.1 First Stage: Compute-Intensive and Efficient Kernels . . . . . . . . . . 835

11.2 Second Stage: Cache-Friendly Computational Kernels . . . . . . . . . 836

11.3 Singular Vectors Computation . . . . . . . . . . . . . . . . . 838

11.4 PLASMA Implementation for Multicore . . . . . . . . . . . . . . 838

11.5 Energy Consumption . . . . . . . . . . . . . . . . . . . . 838

11.6 MAGMA Accelerated Two-Stage Reduction . . . . . . . . . . . . . . 840

11.7 DPLASMA Implementation for Distributed Memory . . . . . . . . . . 841

12 Jacobi Methods $\quad \mathbf{8 4 2}$

12.1 Two-Sided Jacobi SVD . . . . . . . . . . . . . . . . . . . . . . . 844

12.2 One-Sided Jacobi . . . . . . . . . . . . . . . . . . . . . . . 845

12.3 Convergence . . . . . . . . . . . . . . . . . . . . . . 846

12.4 Parallel Orderings . . . . . . . . . . . . . . . . . . . . . 847

12.5 Preconditioning . . . . . . . . . . . . . . . . . . . . . . 848

12.6 Block Jacobi . . . . . . . . . . . . . . . . . . . . . . . . . . . . . . . . . 849

12.7 Performance Analysis . . . . . . . . . . . . . . . . . 850 
$\begin{array}{ll}\text { I4 Additional Test Cases } & 855\end{array}$

$\begin{array}{ll}15 \text { Conclusions } & 858\end{array}$

$\begin{array}{lr}\text { References } & 859\end{array}$

I. Introduction. The singular value decomposition, or SVD, is a very powerful technique for dealing with matrix problems in general. The practical and theoretical importance of the SVD is hard to overestimate, and it has a long and fascinating history. A number of classical mathematicians are associated with the theoretical development of the SVD [107], including Eugenio Beltrami (1835-1899), Camille Jordan (1838-1921), James Sylvester (1814-1897), Erhard Schmidt (1876-1959), and Hermann Weyl (1885-1955).

In recent years, the SVD has become a computationally viable tool for solving a wide variety of problems that arise in many practical applications. The use of the SVD in these applications is centered on the fact that they require information about the rank of a matrix, or a low rank approximation of a matrix, or orthogonal bases for the row and column spaces of a matrix. Applications are as diverse as least squares data fitting [53], image compression [3], facial recognition [111], principal component analysis [92], latent semantic analysis [28], and computing the 2-norm, condition number, and numerical rank of a matrix.

The SVD of an $m$-by- $n$ matrix $A$ is given by

$$
A=U \Sigma V^{T}\left(A=U \Sigma V^{H} \text { in the complex case }\right),
$$

where $U$ and $V$ are orthogonal (unitary) matrices and $\Sigma$ is an $m$-by- $n$ matrix with real diagonal elements, $\sigma_{i}$, conventionally ordered such that

$$
\sigma_{1} \geq \sigma_{2} \geq \cdots \geq \sigma_{\min (m, n)} \geq 0 .
$$

The $\sigma_{i}$ are the singular values of $A$ and the first $\min (m, n)$ columns of $U$ and $V$ are the left and right singular vectors of $A$, respectively.

Theoretically, the SVD can be characterized by the fact that the singular values are the square roots of the eigenvalues of $A^{T} A$, the columns of $V$ are the corresponding eigenvectors, and the columns of $U$ are the eigenvectors of $A A^{T}$, assuming distinct singular values. However, this is not a satisfactory basis for computation because roundoff errors in the formulation of $A^{T} A$ and $A A^{T}$ often destroy pertinent information.

The key to using the SVD is the fact that it can be computed very effectively. There are two dominant categories of SVD algorithms for dense matrices: bidiagonalization methods and Jacobi methods. The classical bidiagonalization method proceeds in the following three stages:

1. The matrix $A$ is reduced to bidiagonal form $A=U_{1} B V_{1}^{T}$ if $A$ is real $(A=$ $U_{1} B V_{1}^{H}$ if $A$ is complex), where $U_{1}$ and $V_{1}$ are orthogonal (unitary if $A$ is complex), and $B$ is real and upper bidiagonal when $m \geq n$ or lower bidiagonal when $m<n$, so that $B$ is nonzero on only the main diagonal and either the first superdiagonal (if $m \geq n$ ) or the first subdiagonal (if $m<n$ ). 
2. The SVD of the bidiagonal matrix $B$ is computed, $B=U_{2} \Sigma V_{2}^{T}$, where $U_{2}$ and $V_{2}$ are orthogonal and $\Sigma$ is diagonal as described above. Several algorithms exist for the bidiagonal SVD, the original being QR iteration.

3. If desired, the singular vectors of $A$ are then computed as $U=U_{1} U_{2}$ and $V=V_{1} V_{2}$.

This is the basic, efficient, and stable algorithm as posed by Golub and Kahan in 1965 [53]. Golub and Reinsch [54] realized the first implementation of the SVD algorithm in Algol60, the programming language of the time. Their paper was later reproduced in the Wilkinson-Reinsch Handbook [117]. Bidiagonalization methods are covered in sections 3 to 11, with additional tests of accuracy and performance on various matrix types in sections 13 and 14 .

In contrast, Jacobi methods apply plane rotations to the entire matrix $A$ without ever reducing it to bidiagonal form. Two-sided Jacobi methods, first proposed by Kogbetliantz in 1955 [76], iteratively apply rotations on both sides of $A$ to bring it to diagonal form, while one-sided Jacobi methods, proposed by Hestenes in 1958 [68], apply rotations on one side to orthogonalize the columns of $A$, implicitly bringing $A^{T} A$ to diagonal. While Jacobi methods are often slower than bidiagonalization methods, there remains interest in them due to their simplicity, easy parallelization, and potentially better accuracy for certain classes of matrices. Jacobi methods are covered in section 12, with additional tests in sections 13 and 14 .

This article traces the development of the SVD algorithm over the past 50 years, using various historical implementations. This development includes algorithmic improvements such as blocking, the divide and conquer (D\&C) and multiple relatively robust representation (MRRR) algorithms, and a two-stage reduction, as well as adapting to new computer architectures such as distributed memory, accelerators, and multicore CPUs. We compare the performance of all the implementations on a common multicore computer. Our focus is on computing all singular values, and optionally, singular vectors, for both square and tall dense matrices. For bisection and MRRR methods we also compute a subset of the singular values and vectors.

2. Experimental Setup. To test the various implementations, we ran six different tests:

1. Square matrices, singular values only (no vectors).

2. Square matrices, singular values and vectors.

3. Tall matrices, $m=3 n$, singular values only (no vectors).

4. Tall matrices, $m=3 n$, singular values and vectors.

5. Tall matrices, $m=1000 n$, singular values only (no vectors).

6 . Tall matrices, $m=1000 n$, singular values and vectors.

When computing singular vectors, we computed the reduced $S V D$ consisting of the first $\min (m, n)$ columns of $U$ and $V$ and $\min (m, n)$ rows and columns of $\Sigma$. This is the most useful part computationally, sufficient for many applications such as solving least squares problems, and we subsequently identify $U, V$, and $\Sigma$ with those of the reduced SVD, which still satisfy (1). For LAPACK, the reduced SVD corresponds to job="s" for both $U$ and $V$. We store $U$ and $V$ separately from $A$, i.e., they do not overwrite $A$. Where applicable, we query for the optimal workspace size; otherwise, we use the maximum documented workspace size. This ensures that we always use the "fast" path in codes, including blocking and other optimizations.

Unless indicated, matrices have random entries from a uniform distribution on $(0,1)$. For some tests, we generate singular values $\Sigma$ according to one of the distribu- 
tions below, then form $A=U \Sigma V^{T}$, where $U$ and $V$ are random orthogonal matrices from the Haar distribution [106]. Where given, $\kappa$ is the condition number of $A$.

- $\Sigma$ random: singular values are random uniform on $(0,1)$. The condition number is not determined a priori.

- Arithmetic: $\sigma_{i}=1-\frac{i-1}{n-1}\left(1-\frac{1}{\kappa}\right)$ for $i=1, \ldots, n$.

- Geometric: $\sigma_{i}=\kappa^{-(i-1) /(n-1)}$ for $i=1, \ldots, n$.

- Log-random: singular values are random in $\left(\frac{1}{\kappa}, 1\right)$ such that their logarithms are random uniform on $\left(\log \frac{1}{\kappa}, \log 1\right)$.

- Cluster at $\frac{1}{\kappa}: \Sigma=\left[1, \frac{1}{\kappa}, \ldots, \frac{1}{\kappa}\right]$.

- Cluster at 1: $\Sigma=\left[1, \ldots, 1, \frac{1}{\kappa}\right]$.

All tests were performed in double-precision real arithmetic. Except for PLASMA and MPI-based implementations, which initialize memory in parallel, we used numact 1 --interleave=all to distribute memory across CPU sockets, and the CPU cache was flushed before the SVD function call. To avoid repeating minor differences, we shall generally assume that $A$ is real and $m \geq n$. Operations for complex or $m<n$ are analogous.

We conducted experiments on a two-socket Intel Sandy Bridge Xeon E5-2670 running at $2.6 \mathrm{GHz}$, with 8 cores per socket, a theoretical double-precision peak of $333 \mathrm{Gflop} / \mathrm{s}$, and $64 \mathrm{GiB}$ of main memory. The measured practical dgemm peak is 313.6 Gflop/s and dgemv peak is 13.9 Gflop/s $(55.8 \mathrm{~GB} / \mathrm{s})$. The STREAM triad benchmark [91] measured the memory bandwidth as $57.8 \mathrm{~GB} / \mathrm{s}$ with 16 OpenMP threads. All CPU implementations were compiled with gcc and linked against Intel's Math Kernel Library (MKL) version 11.2.3 [71].

GPU results used an NVIDIA Kepler K40c with 15 multiprocessors, each containing 192 CUDA cores. The theoretical double-precision peak performance is 1682 Gflop/s. On the GPU, 12 GiB of device memory can be accessed at a theoretical bandwidth of $288 \mathrm{~GB} / \mathrm{s}$. The measured practical dgemm peak is $1243.1 \mathrm{Gflop} / \mathrm{s}$ and dgemv peak is $45.3 \mathrm{Gflop} / \mathrm{s}(181.2 \mathrm{~GB} / \mathrm{s})$. For the GPU implementation, we used CUDA version 7.0 [94].

3. EISPACK Implementation. The EISPACK project was an effort to develop a software library for numerical computation of eigenvalues and eigenvectors of matrices based on algorithms and ideas that were mainly contained in the WilkinsonReinsch Handbook [117]. EISPACK was a transliteration of these Algol programs into Fortran. It contains subroutines for calculating the eigenvalues of nine classes of matrix problems: complex general, complex Hermitian, real general, real symmetric, real symmetric banded, real symmetric tridiagonal, special real tridiagonal, generalized real, and generalized real symmetric. In addition, it includes subroutines to perform an SVD [50]. Some routines were updated to implement improvements in the numerical accuracy and achieve portability across different computing systems. However, the basic organization and access to matrix elements was kept in the Algol style.

To arrange multidimensional arrays in linear storage such as memory, Algol uses row-major order (each row is contiguous in memory), while Fortran uses columnmajor order (each column is contiguous in memory). Array layout is critical for correctly passing arrays between programs written in different languages. It is also important for performance when traversing an array, since accessing array elements that are contiguous in memory is usually much faster than accessing elements that are not, due to the structure of the memory cache hierarchy. In the Algol routines, and subsequently the Fortran routines of EISPACK, matrix elements were referenced 


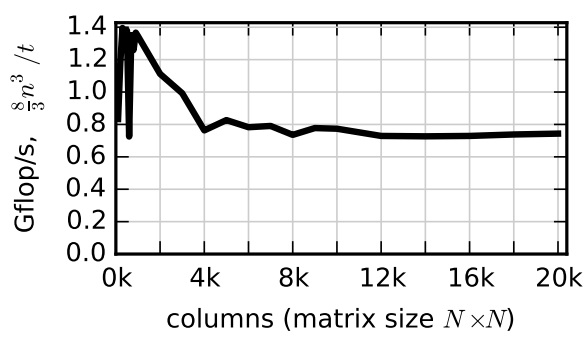

(a) square, no vectors

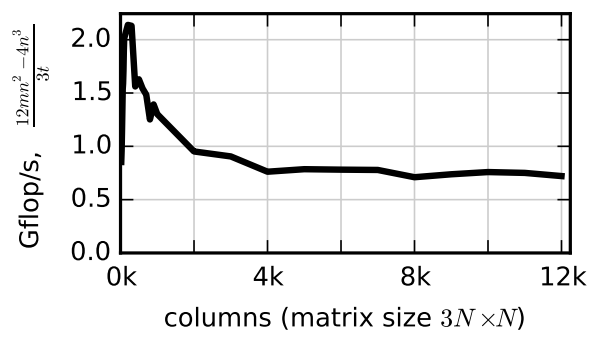

(c) tall, 3:1, no vectors

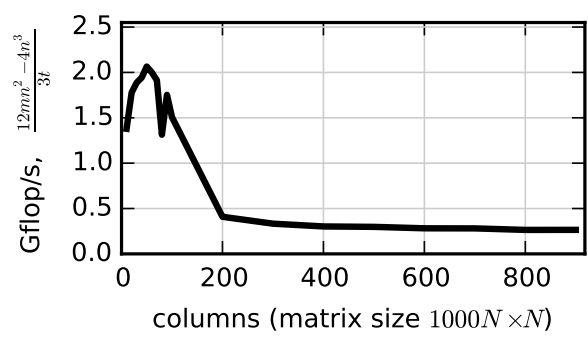

(e) tall, 1000:1, no vectors

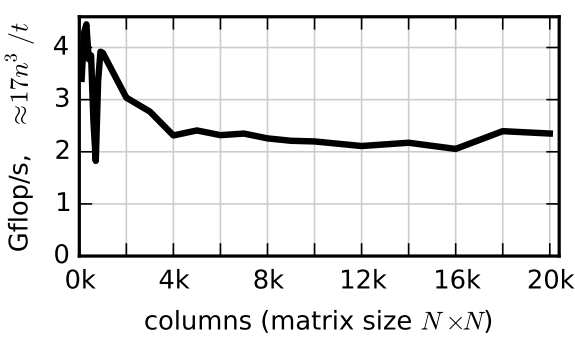

(b) square, vectors

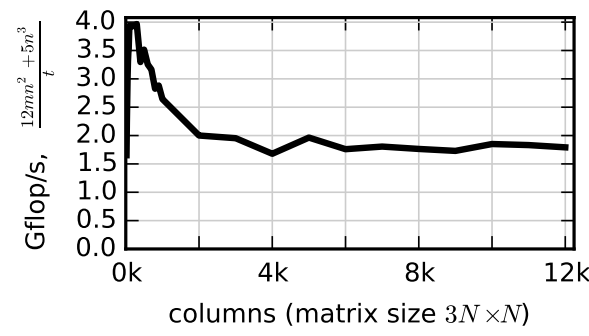

(d) tall, 3:1, vectors

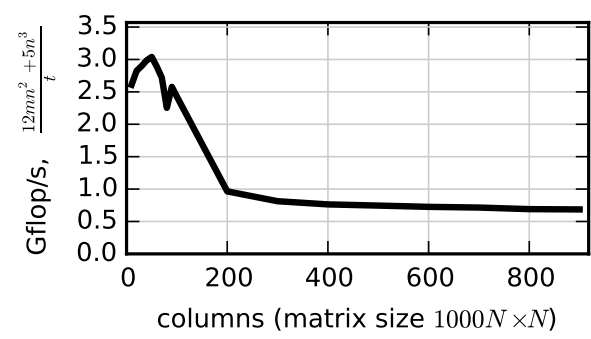

(f) tall, 1000:1, vectors

Fig. I Results for EISPACK, which uses only one core.

by row, thus causing great inefficiencies in the Fortran EISPACK software on modern cache-based computer systems.

Written in standard Fortran 77, with no outside dependencies, EISPACK still compiles with a modern Fortran compiler. Figure 1 shows its performance results on a modern computer for the six test problems described in section 2. EISPACK has no notion of parallelism, so the code runs on only a single core. The operation count formulas here assume two QR iterations per singular value, and that an initial QR reduction is not done [23].

For square matrices without computing singular vectors, asymptotic performance is limited to $0.74 \mathrm{Gflop} / \mathrm{s}$ for one core, while when computing singular vectors, performance nearly triples to $2.17 \mathrm{Gflop} / \mathrm{s}$. As is common, small sizes perform better because the entire matrix fits into L2 cache. Performance for the tall 3:1 and 1000:1 cases is less than the square case, but exhibits a similar improvement when computing singular vectors compared with no vectors. For comparison, the practical peak using matrix-multiply on one core is $20 \mathrm{Gflop} / \mathrm{s}$. 
4. LINPACK Implementation Using BLAS. In the 1970s, the Level 1 BLAS (Basic Linear Algebra Subroutines) [79] were introduced as a standard set of interfaces to perform common linear algebra operations. The Level 1 BLAS includes operations with $O(n)$ floating-point operations (flops), such as vector sum $(y=\alpha x+y$, called daxpy). The LINPACK project [39] reimplemented the SVD algorithm, along with other linear algebra algorithms, using Level 1 BLAS for efficient execution on the vector supercomputers of the 1970s and 1980s. It uses Fortran's native column-major order, which makes better use of cache and memory bandwidth. However, using Level 1 BLAS, LINPACK is limited by the memory bandwidth and receives little benefit from multiple cores. We see in Figure 2 that LINPACK achieves up to $3.9 \times$ speedup over EISPACK for the square, no vectors case, and $2.7 \times$ speedup for the square, vectors case. When computing a tall $m \times n$ matrix with $m=1000 n$, using multithreaded BLAS on 16 cores yields some benefit, with speedups of $22.5 \times$ and $13.5 \times$ over EISPACK for the no vectors and vectors cases, respectively, compared with speedups of $7.6 \times$ and $3.9 \times$, respectively, with single-threaded BLAS. In some instances, for large matrices such as $n=16,000$, the code hung, appearing in a "sleep" state in ps, so we were unable to collect all data points.

5. LAPACK Implementation Based on Blocked Householder Transformations. While successful for vector-processing machines, Level 1 BLAS were not a good fit for the cache-based machines that emerged later in the 1980s. For cachebased machines, it is preferable to use higher-level operations such as matrix-matrix multiply, which is implemented by splitting a matrix into small blocks that fit into cache memory and performing small matrix-matrix multiplies on these blocks. This avoids excessive data movement between cache and main memory. This led to the Level 2 BLAS [41] for operations with $O\left(n^{2}\right)$ flops, such as general matrix-vector multiply ( $y=\alpha A x+\beta y$, called dgemv); and Level 3 BLAS [40] for operations with $O\left(n^{3}\right)$ flops on $O\left(n^{2}\right)$ data, such as general matrix-matrix multiply $(C=\alpha A B+\beta C$, called dgemm). Level 1 and 2 BLAS access $O(1)$ elements per operation, and are thus limited in performance by the memory bandwidth. Level 3 BLAS benefit from the surface-to-volume effect of having only $O\left(n^{2}\right)$ elements to access for $O\left(n^{3}\right)$ operations. The performance of Level 1, 2, and 3 BLAS are compared in Figure 3, showing the significant benefit of Level 3 BLAS. The BLAS provide a means to write high-level, high-performance, portable numerical software. Optimized BLAS libraries are available, from commercial vendors such as the Intel Math Kernel Library (MKL) [71] and the IBM Engineering and Scientific Subroutine Library (ESSL) [70], and also in open-source libraries such as OpenBLAS [96] and ATLAS [115]. These math libraries often also included optimized versions of LAPACK, ScaLAPACK, and other numerical libraries. Our tests used the optimized routines available in Intel MKL.

5.I. Blocked Householder Transformations. With the introduction of Level 3 BLAS, algorithms were recast using matrix multiplies, and LINPACK was redesigned into LAPACK [2] to use Level 3 BLAS where possible. The redesign for one-sided factorizations such as QR, LU, and Cholesky is relatively easier than reductions for eigenvalue problems and the SVD because the transformations used in QR, LU, and Cholesky are applied from only the left side [40]. Consecutive elementary transformations are restricted to a block of columns at a time, referred to as the panel (depicted in Figure 4(a)), and updates to the rest of the matrix, referred to as the trailing matrix, are delayed. The transformations used for a panel are blocked together $[14,104]$ and applied to the trailing matrix as Level 3 BLAS. 


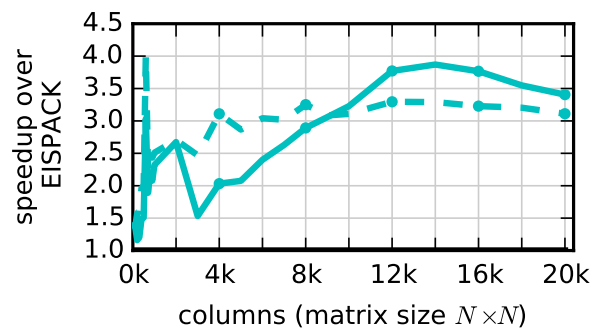

(a) square, no vectors

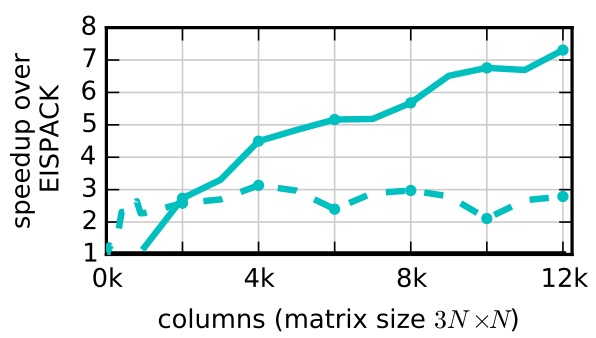

(c) tall, 3:1, no vectors

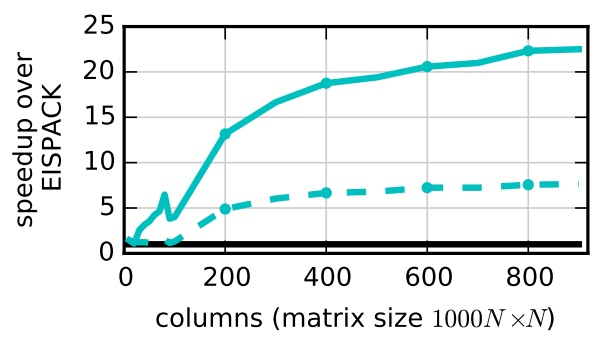

(e) tall, 1000:1, no vectors

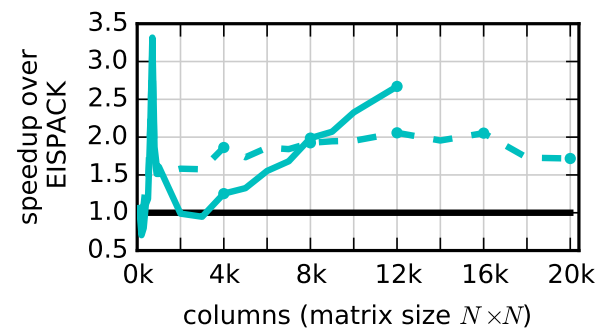

(b) square, with vectors

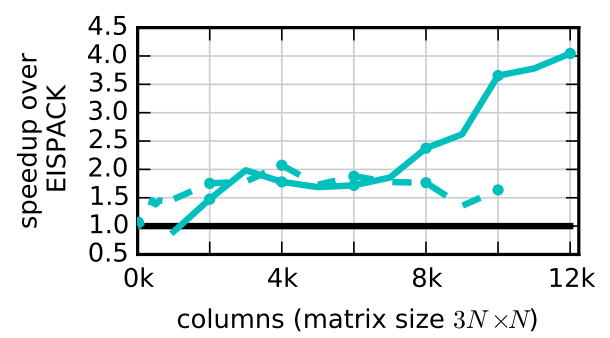

(d) tall, 3:1, with vectors

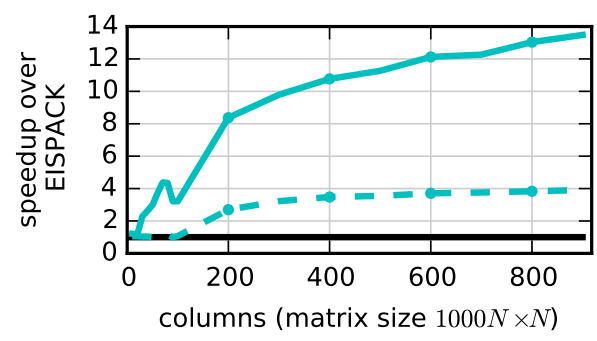

(f) tall, 1000:1, with vectors

$$
\because \text { LINPACK } \quad \longrightarrow \text { EISPACK (1 core) }
$$

Fig. 2 Comparison of LINPACK to EISPACK.

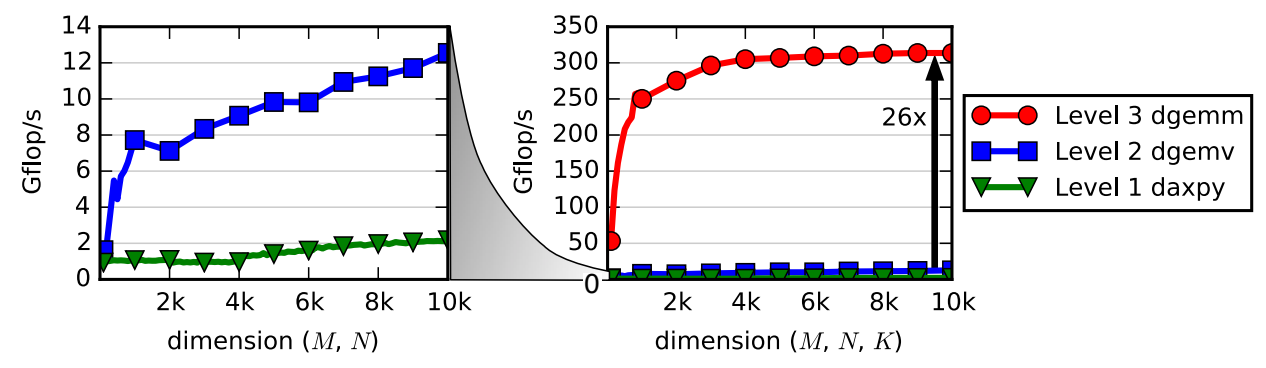

Fig. 3 Comparison of Level 1, 2, and 3 BLAS performance. 


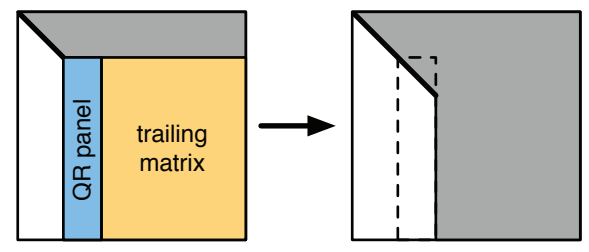

(a) QR factorization

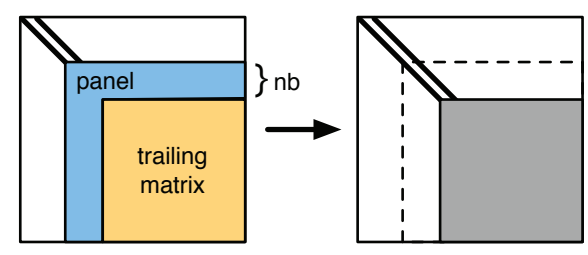

(b) Bidiagonal reduction

Fig. 4 Comparison of panels and trailing matrix.

On the other hand, the reduction of a matrix $A$ to bidiagonal form is done by applying orthogonal matrices on both the left and right sides of $A$ - hence it is called a "two-sided factorization." The two-sided transformations create more data dependencies, which make it impossible to entirely remove matrix-vector products involving the trailing matrix (as in the one-sided factorizations). The panel becomes a block row and block column, as shown in Figure 4(b), but panel operations also involve the entire trailing matrix. Dongarra, Sorensen, and Hammarling [42] developed the blocked algorithm for the bidiagonal reduction. The algorithm as implemented in LAPACK is given in Algorithm 1, and can be summarized as follows.

Two orthogonal matrices, $U_{1}$ and $V_{1}$, are applied on the left and right sides, respectively, of an $m \times n$ matrix $A$ to reduce it to bidiagonal form, $B=U_{1}^{T} A V_{1}$. The matrices $U_{1}$ and $V_{1}$ are represented as products of elementary Householder reflectors:

$$
U_{1}=H_{1} H_{2} \ldots H_{n} \quad \text { and } \quad V_{1}=G_{1} G_{2} \ldots G_{n-1} .
$$

Each $H_{i}$ and $G_{i}$ has the form

$$
H_{i}=I-\tau_{i} v_{i} v_{i}^{T} \quad \text { and } \quad G_{i}=I-\pi_{i} u_{i} u_{i}^{T},
$$

where $\tau_{i}$ and $\pi_{i}$ are scalars, and $v_{i}$ and $u_{i}$ are vectors. $H_{i}$ eliminates elements below the diagonal in column $i$, while $G_{i}$ eliminates elements right of the superdiagonal in row $i$. Let $A_{(i-1)}$ be the reduced matrix $A$ after step $i-1$. Applying $H_{i}$ on the left yields

$$
H_{i} A_{(i-1)}=\left(I-\tau_{i} v_{i} v_{i}^{T}\right) A_{(i-1)}=A_{(i-1)}-v_{i} y_{i}^{T},
$$

while applying both $H_{i}$ and $G_{i}$ yields

$$
\begin{aligned}
A_{(i)}=H_{i} A_{(i-1)} G_{i} & =\left(I-\tau_{i} v_{i} v_{i}^{T}\right) A_{(i-1)}\left(I-\pi_{i} u_{i} u_{i}^{T}\right) \\
& =A_{(i-1)}-v_{i} y_{i}^{T}-x_{i} u_{i}^{T},
\end{aligned}
$$

where $y_{i}=\tau_{i} A_{(i-1)}^{T} v_{i}$ and $x_{i}=\pi_{i}\left(A_{(i-1)}-v_{i} y_{i}^{T}\right) u_{i}$. Blocking together $i$ applications of (3), we obtain

$$
A_{i}=H_{i} \cdots H_{1} A G_{1} \cdots G_{i}=A-V_{i} Y_{i}^{T}-X_{i} U_{i}^{T},
$$

where $U_{i}=\left[u_{1}, \ldots, u_{i}\right]$, and similarly with $V_{i}, X_{i}$, and $Y_{i}$. Note that it is possible to update just part of $A$, namely, the $i$ th column and row of $A$, in order to proceed with the computation of the $H_{i}$ and $G_{i}$. Thus, a delayed update is possible, but at each step we still compute two matrix-vector products involving the entire trailing matrix of $A$. As a result, if $m=n$, the entire factorization takes approximately $\frac{8}{3} n^{3}$ flops, with half of the operations in Level 2 BLAS (matrix-vector products), while the other half are in Level 3 BLAS. 


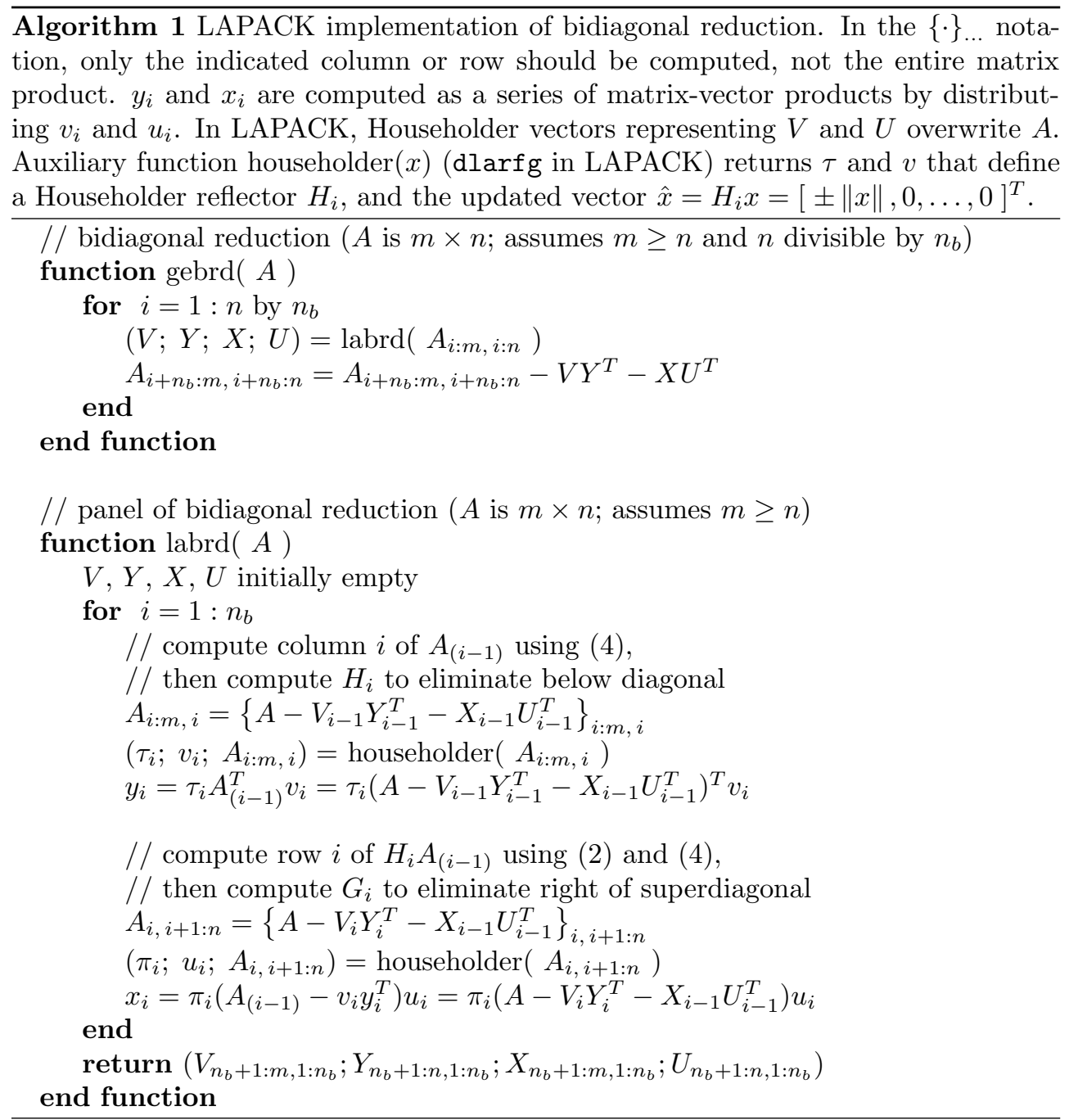

5.2. QR Iteration. After the bidiagonal reduction, LAPACK solves the bidiagonal SVD using QR iteration, similar to EISPACK and LINPACK, or using divide and conquer $(\mathrm{D} \& \mathrm{C})$, which is described later in section 7. The original QR iteration algorithm computed singular values to high absolute accuracy, meaning small singular values might be inaccurate. Demmel and Kahan [31] derived the implicit zero-shift QR iteration algorithm and proved that it computes all singular values to high relative accuracy; this is used as needed for accuracy by LAPACK when computing singular vectors. Accuracy is discussed further in section 13.

The qd (German: quotienten-differenzen) [100] and differential qd (dqd) [101] algorithms proposed by Rutishauser actually predate QR iteration and are among the first algorithms for computing singular values for modern computers. Subsequent to Demmel and Kahan's work, Fernando and Parlett [48] derived a shifted version called dqds that allowed the use of shifts to maintain fast convergence, while still maintaining high relative accuracy. This is used by LAPACK when computing singular values only (no vectors). Quite a few more variants of qd can be derived [97]. 
5.3. Computation of Singular Vectors. Normally, LAPACK stores orthogonal matrices in an implicit fashion as a sequence of Householder reflectors, each represented by a scalar $\tau_{i}$ and vector $u_{i}$. For QR iteration to accumulate the singular vectors, it first generates $U_{1}$ and $V_{1}$ explicitly (using dorgbr); this is essentially applying block Householder reflectors to an identity matrix as a series of Level 3 BLAS operations.

The QR iteration algorithm then updates $U_{1}$ and $V_{1}$ by applying the Givens rotations used to reduce the bidiagonal matrix to diagonal. This is implemented in a Level 2 BLAS-like fashion, where an entire sequence of $n$ Givens rotations is applied to update the entire $U$ and $V$ matrices (using dlasr). Recently, Van Zee, Van de Geijn, and Quintana-Ortí [113] developed a Level 3 BLAS-like implementation of applying Givens rotations, which they found made the SVD using QR iteration competitive with the SVD using D\&C (discussed in section 7).

5.4. Initial QR Factorization. If $m \gg n$, it is more efficient to first perform a $\mathrm{QR}$ factorization of $A$ and then compute the SVD of the $n$-by- $n$ matrix $R$, since if $A=Q R$ and $R=U \Sigma V^{T}$, then the SVD of $A$ is given by $A=(Q U) \Sigma V^{T}$. Similarly, if $m \ll n$, it is more efficient to first perform an LQ factorization of $A$. Chan [23] analyzed this optimization, showing that it reduces the number of floating-point operations. The operation counts are given in Table 1, with the theoretical crossover points based on flops. Figure 5 plots the operation count as the ratio $m: n$ increases, illustrating the large savings as a matrix becomes taller. The results for tall matrices in Figures 7(c) to 7 (f) show that LAPACK achieves significant speedups, such as $120 \times$ compared with EISPACK. This is a result of the reduced operation count and the fact that much of the computation is done via Level 3 BLAS in QR factorization, followed by a relatively small square SVD problem.

Table I Floating-point operation counts.

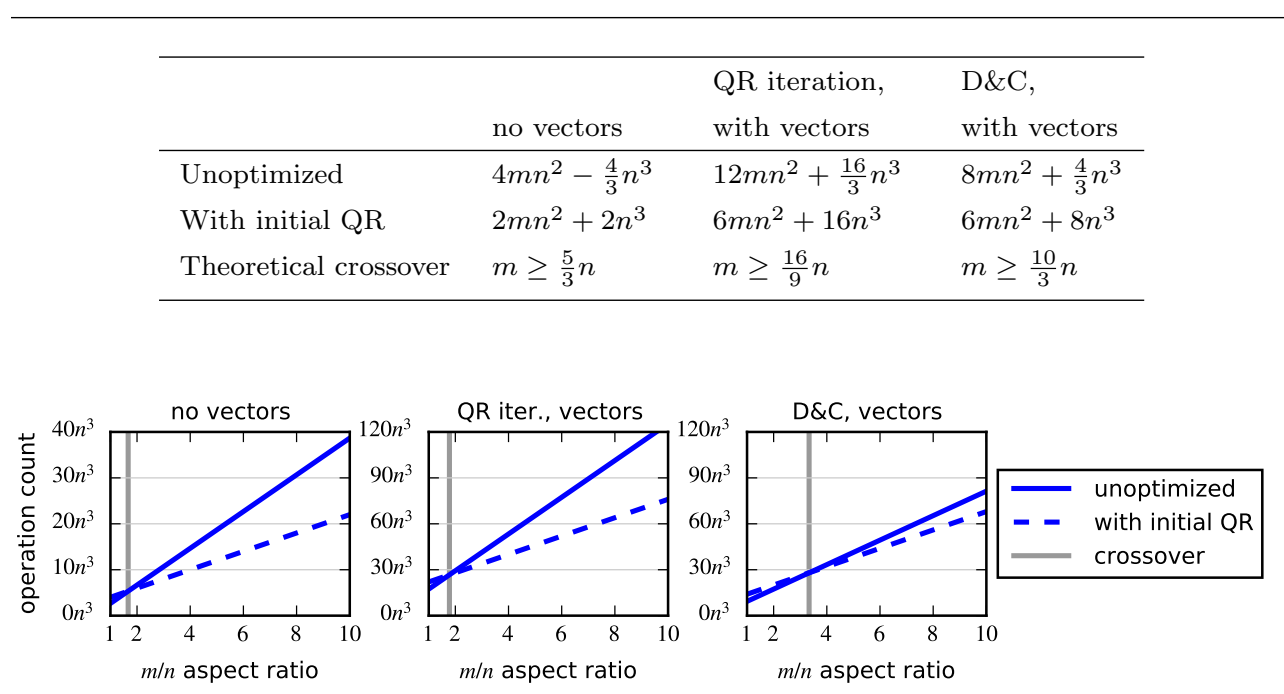

Fig. 5 Operation counts as ratio $m: n$ increases (i.e., matrix gets taller), showing crossover where doing initial $Q R$ factorization is beneficial.

Since bidiagonal D\&C (discussed in section 7) always operates on a square matrix, doing an initial QR factorization with D\&C results in less of an improvement than with 

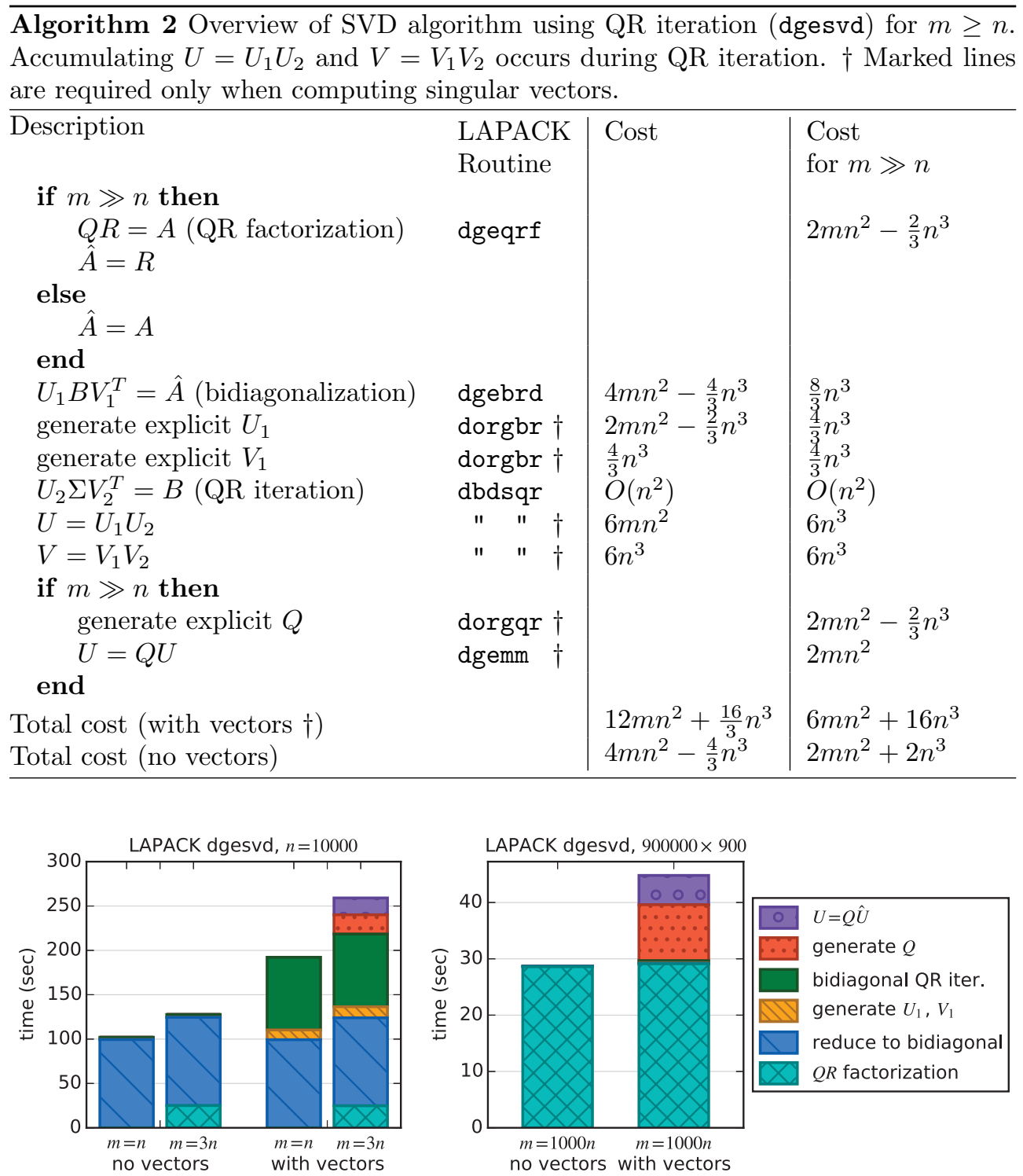

Fig. 6 Profile of LAPACK SVD. Left are $10000 \times 10000$ and $30000 \times 10000$ problems. QR factorization reduces the $30000 \times 10000$ matrix to a $10000 \times 10000$ matrix. Right is a $900000 \times 900$ problem, where reduction to bidiagonal and $Q R$ iteration become vanishingly small.

QR iteration. Asymptotically, as the ratio $m: n \rightarrow$ inf, the initial QR factorization, generating $Q$, and multiplying by $Q$ are responsible for most of the cost, as is shown by the profile of the 1000:1 case in Figure 6. As a result, using QR iteration or D\&C yields the same performance for very tall-skinny matrices. The crossover points in Table 1 are based solely on flop counts. Since doing an initial QR also shifts operations from Level 2 to Level 3 BLAS, the crossovers are ideally tunable parameters, for instance, by using LAPACK's ilaenv tuning function. 
5.5. Results. An overview of all the phases in the complete SVD is given in Algorithm 2, with a profile of the time spent in each phase in Figure 6. For the square, no vectors case, we see that the bidiagonal reduction (blue with $\backslash \backslash$ hatching) takes almost the entire time, while QR iteration (green, no hatching) takes very little time, as is expected since QR iteration is $O\left(n^{2}\right)$ while the bidiagonal reduction costs $\frac{8}{3} n^{3}$ flops. When computing singular vectors, the $\mathrm{QR}$ iteration time becomes nearly half of the overall time, due to accumulating $U=U_{1} U_{2}$ and $V=V_{1} V_{2}$. Generating the explicit $U_{1}$ and $V_{1}$ matrices (orange with $\backslash \backslash \backslash \backslash$ hatching) is a small portion of the time, even though together they have nominally the same operation count $\left(\frac{8}{3} n^{3}\right)$ as the bidiagonal reduction. This exemplifies the performance difference between Level 2 BLAS, in the bidiagonal reduction, and Level 3 BLAS, in generating $U_{1}$ and $V_{1}$.

The tall 3:1 matrix first does an initial QR factorization, resulting in a square $R$ matrix the same size as the square case $(10000 \times 10000)$. Thus, the profile for the $3: 1$ case simply adds the QR factorization, generating $Q$, and multiplying $U=Q \hat{U}$ steps to the square case. For the tall 1000:1 matrix, the initial QR factorization dominates the overall time, with the subsequent bidiagonal reduction and $\mathrm{QR}$ iteration becoming vanishingly small. When vectors are computed, generating $Q$ and multiplying $U=$ $Q \hat{U}$ add significant time, while generating $U_{1}$ and $V_{1}$ and updating $U=U_{1} U_{2}$ and $V=V_{1} V_{2}$ during $\mathrm{QR}$ iteration are also vanishingly small. Thus, for very tall-skinny matrices, the performance is dominated by operations rich in Level 3 BLAS.

Figure 7 shows the speedup that LAPACK achieves compared with EISPACK. Even a single-core implementation may achieve over $5 \times$ speedup. But the real potential is shown when using multiple cores (16 in this case) - a $45 \times$ speedup is possible for square matrices and over $350 \times$ speedup for tall matrices with a 1000:1 row-to-column ratio. The square, no vectors case in Figure 7 (a) is dominated by the bidiagonalization, as the subsequent bidiagonal SVD is $O\left(n^{2}\right)$. With Level 3 BLAS being significantly faster than Level 2 BLAS, and half the operations in Level 2 BLAS, we expect the bidiagonalization to be about $2 \times$ the speed of Level 2 BLAS. Modeling the time as

$$
t=\frac{4 n^{3}}{3 r_{2}}+\frac{4 n^{3}}{3 r_{3}}
$$

with the Level 2 BLAS rate as $r_{2}=13.4 \mathrm{Gflop} / \mathrm{s}$ and the Level 3 BLAS rate as $r_{3}=315$ Gflop $/ \mathrm{s}$ (from Figure 3), we obtain a theoretical bound of 25.7 Gflop/s. This yields a speedup of $34.7 \times$ over EISPACK - exactly what we see for LAPACK in Figure 7(a). When computing singular vectors, LAPACK achieves a higher speedup, up to $45.3 \times$, reflecting that computation of $U_{1}$ and $V_{1}$ uses Level 3 BLAS. The tall matrix cases achieve even higher speedups because much of the work is done in the initial QR factorization.

5.6. Level 2.5 BLAS Implementation. Since the bidiagonalization performance is limited by the Level 2 BLAS operations, Howell et al. [69] sought to optimize these operations by observing that several Level 2 operations can be done together, thus reducing memory transfers by keeping data in cache. This technique of fusing several Level 2 operations together was called the Level 2.5 BLAS [69, 17]. For instance, to compute

$$
\begin{aligned}
x & =\beta A^{T} y+z, \\
w & =\alpha A x,
\end{aligned}
$$

known as dgemvt, $A$ is partitioned into block columns as

$$
A=\left[A_{1} A_{2} \cdots A_{k}\right]
$$




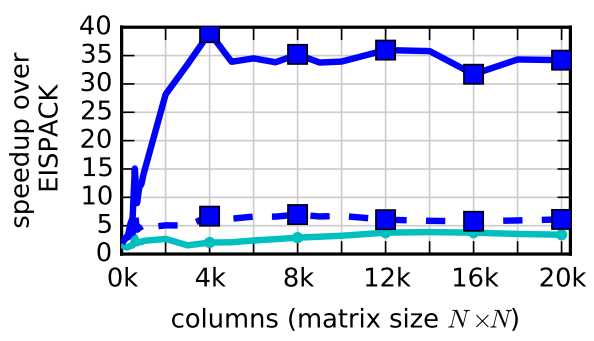

(a) square, no vectors

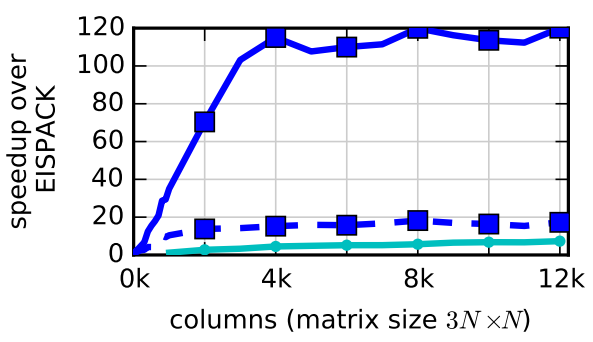

(c) tall 3:1, no vectors

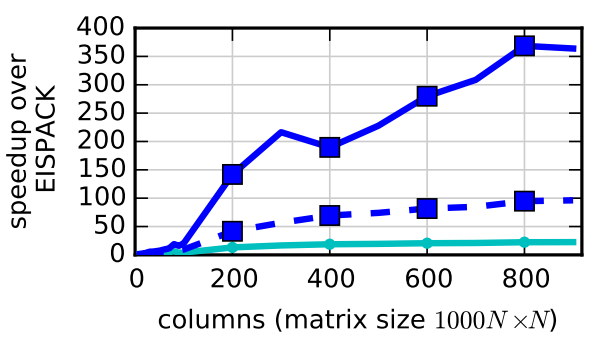

(e) tall 1000:1, no vectors

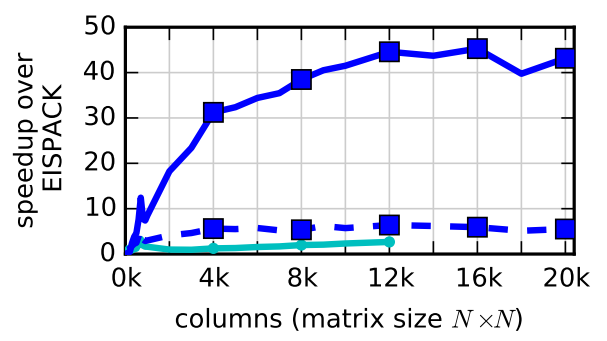

(b) square, with vectors

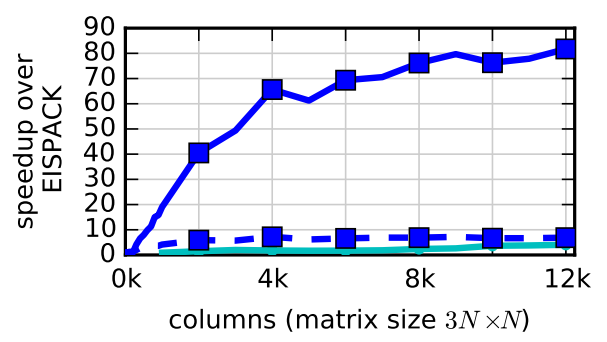

(d) tall 3:1, with vectors

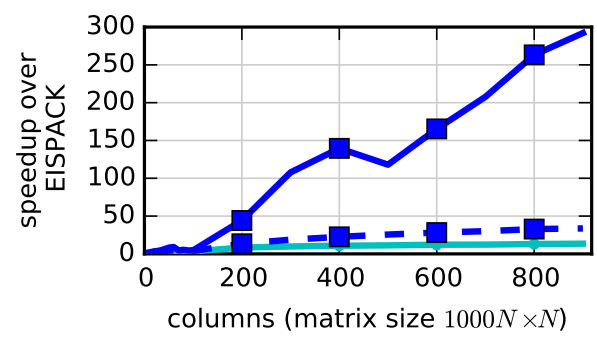

(f) tall 1000:1, with vectors

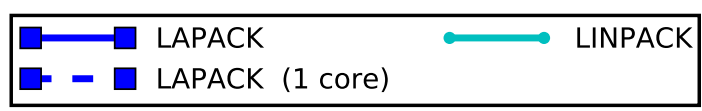

Fig. 7 Comparison of LAPACK, LINPACK, and EISPACK. Solid lines represent 16-core runs; dashed lines represent single-core runs.

where each $A_{i}$ has $b$ columns and is sized such that it fits into cache. Correspondingly, $x$ and $z$ are partitioned as

$$
x=\left[\begin{array}{c}
x_{1} \\
\vdots \\
x_{k}
\end{array}\right], \quad z=\left[\begin{array}{c}
z_{1} \\
\vdots \\
z_{k}
\end{array}\right] .
$$

The dgemvt loops over the $A_{i}$ blocks, performing two dgemv operations with each block as shown in Algorithm 3. Keeping each $A_{i}$ in cache for the second dgemv cuts main memory traffic roughly in half, thereby increasing the potential performance. With some algebraic manipulation, the two products $y_{i}=\tau_{i} A^{T} v_{i}$ and $x_{i}=\pi_{i} A u_{i}$ 


\begin{tabular}{|c|c|c|c|c|c|}
\hline 1,1 & 1,2 & 1,3 & 1,4 & 1,5 & 1,6 \\
\hline 2,1 & 2,2 & 2,3 & 2,4 & 2,5 & 2,6 \\
\hline 3,1 & 3,2 & 3,3 & 3,4 & 3,5 & 3,6 \\
\hline 4,1 & 4,2 & 4,3 & 4,4 & 4,5 & 4,6 \\
\hline
\end{tabular}

(a) Global view of matrix. Each square is an $n_{b} \times n_{b}$ block, colored by the process that it is distributed to in (b).

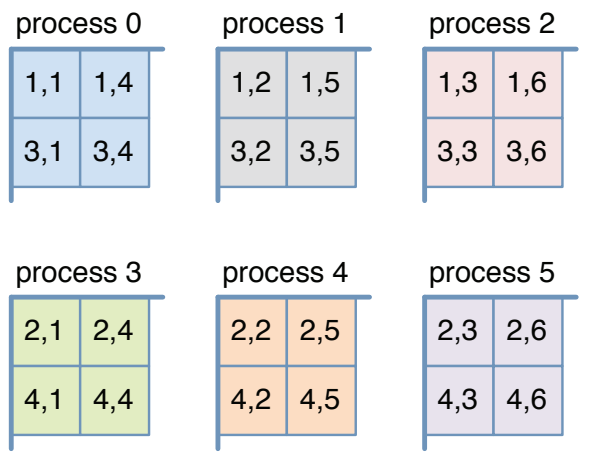

(b) Local view of matrix. Each local submatrix is stored in column-major order.

Fig. $82 D$ block cyclic distribution of the matrix $A$ using 2-by-3 processor grid.

from the bidiagonalization panel can be computed together using this dgemvt. Tests that Howell et al. [69] ran showed a $1.2-1.3 \times$ speedup over the existing LAPACK implementation for the bidiagonalization. Van Zee et al. [114] further analyzed these operations and fused them at the register level, reusing data in registers to also avoid unnecessary accesses to cache memory, showing potential further speedups. So far, these results have been for single-threaded implementations, and the speedups do not carry over when using multithreaded BLAS. If optimized Level 2.5 BLAS becomes available for multicore processors, this might become a viable approach, but we don't pursue it further here.

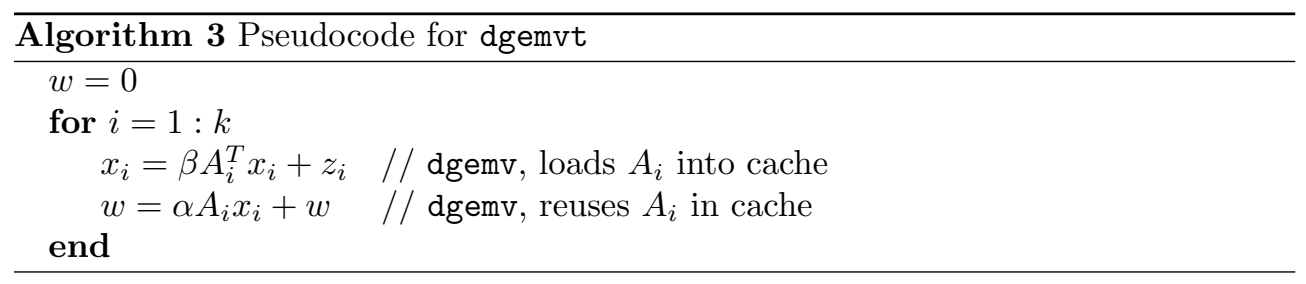

6. ScaLAPACK Implementation. To use a distributed-memory computer, the Scalable Linear Algebra Package (ScaLAPACK) [16] extends LAPACK by distributing the matrices in a 2D block cyclic layout using the prescribed block size $n_{b}$ and the pair of parameters $(p, q)$ to define a $p$-by- $q$ process grid, as illustrated in Figure 8. ScaLAPACK parallelizes the LAPACK subroutines using the parallel version of BLAS (PBLAS) and the Basic Linear Algebra Communication Subprograms (BLACS) for the interprocess communication, implemented on top of the Message Passing Interface (MPI) [93]. For instance, to bidiagonalize the input matrix for computing the SVD [24], dgebrd of LAPACK uses the Level 2 BLAS matrix-vector multiply (dgemv) to perform about half of its total flops. Now, to perform the matrix-vector multiply on a distributed-memory computer, in pdgemv of PBLAS, each process first gathers all the required nonlocal block rows of the input vector from other processes. After the completion of this initial interprocess communication, each process independently 


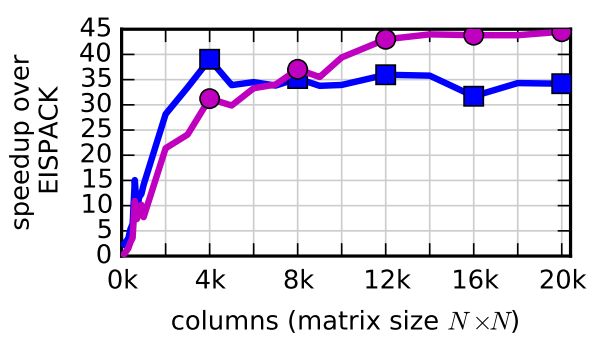

(a) square, no vectors

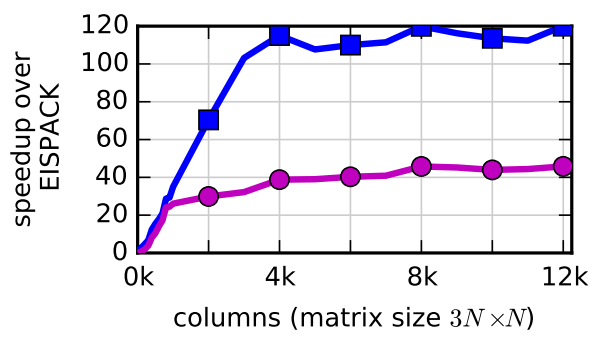

(c) tall, 3:1, no vectors

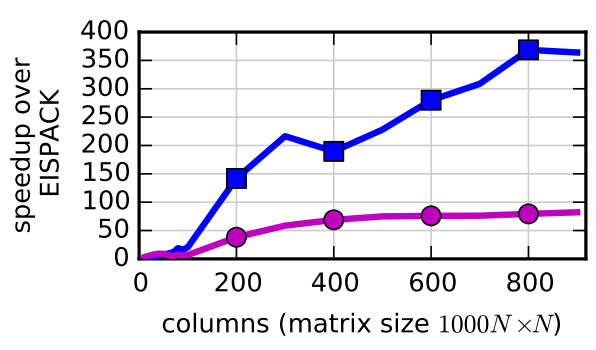

(e) tall, 1000:1, no vectors

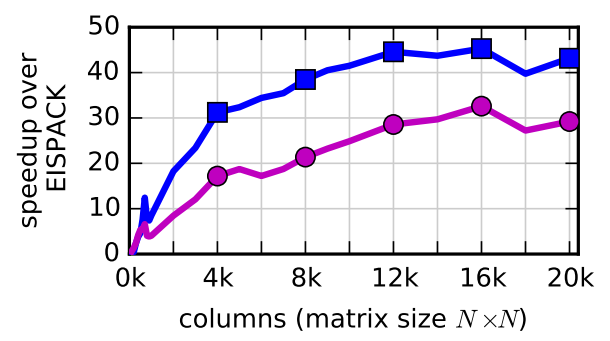

(b) square, with vectors

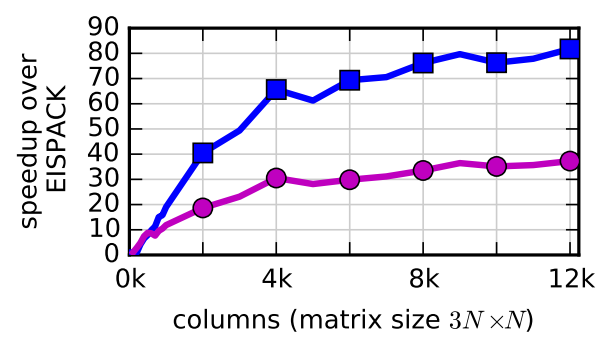

(d) tall, 3:1, with vectors

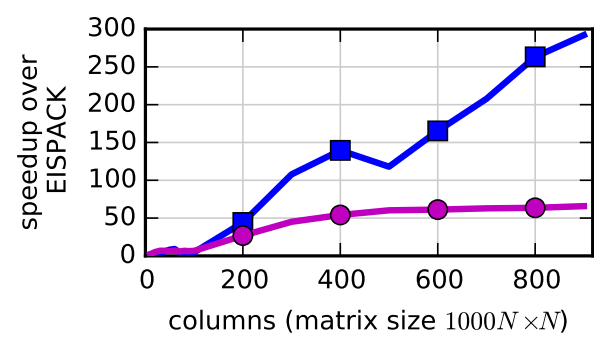

(f) tall, 1000:1, with vectors

0 ScaLAPACK $\square$ LAPACK

Fig. 9 Comparison of ScaLAPACK to LAPACK.

computes the matrix-vector multiplication with the local submatrix. Finally, each process computes the local part of the output vector by gathering and accumulating the partial results from the other processes in the same row of the process grid. Hence, ScaLAPACK follows the fork-join parallel programming paradigm and is designed for weak parallel scalability of the algorithm. Since PBLAS performs most of its local computation using BLAS, ScaLAPACK can exploit a NUMA (nonuniform memory access) architecture using a threaded version of BLAS.

Figure 9 compares the performance of ScaLAPACK's pdgesvd with the performance of LAPACK's threaded dgesvd for computing the SVD on our 16-core sharedmemory computer. While, from ScaLAPACK's perspective, each MPI process has its own memory and explicit messages are passed between MPI processes, on a sharedmemory computer the MPI implementation uses an efficient shared-memory communication layer to copy data. See section 11 for ScaLAPACK's performance on a 


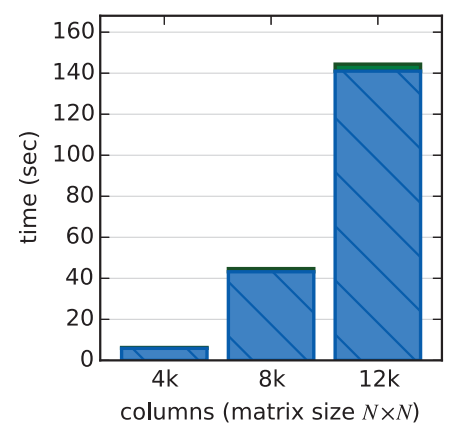

(a) square, no vectors

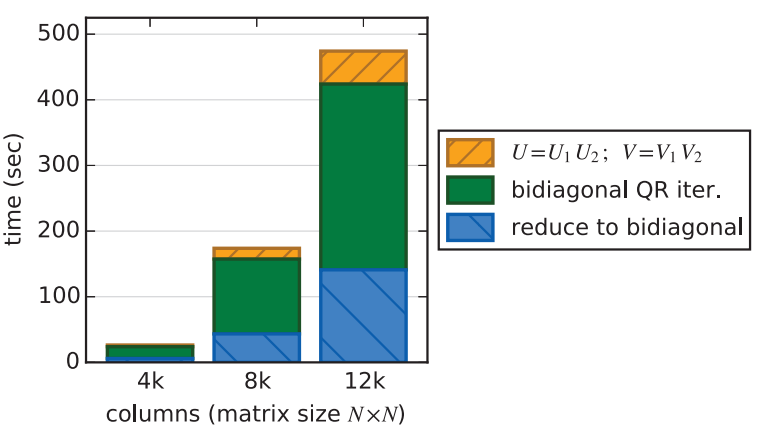

(b) square, with vectors

Fig. 10 Profile of ScaLAPACK reference implementation with $n_{b}=32$ and $(p, q)=(4,4)$.

distributed-memory computer. The performance of pdgesvd was obtained using the tester included in ScaLAPACK version 2.0.2, which was linked with ScaLAPACK and the sequential LAPACK/BLAS of Intel MKL. We tested the performance of pdgesvd using 8 -by-2, 4-by- 4 , and 2-by- 8 processor grids and block sizes of 32,64 , and 128 . The figure shows the optimal performance among these parameter configurations. We see that the performance of pdgesvd was often lower than the performance of LAPACK's dgesvd. This is mainly because several optimizations have not been implemented in pdgesvd. For instance, for a tall-skinny matrix $(m \gg n)$, dgesvd computes the QR factorization of the input matrix $A$, followed by the SVD of the resulting uppertriangular matrix, as described in subsection 5.4. For a tall-skinny matrix $A$, this greatly reduces the number of required floating-point operations compared to that of pdgesvd, which directly computes the SVD of the input matrix. As a result, for computing the SVD of a tall-skinny matrix, pdgesvd was slower than dgesvd.

After the bidiagonalization of the input matrix $A$, pdgesvd computes the SVD of the bidiagonal matrix using dbdsqr of LAPACK. If only the singular values are requested, pdgesvd typically spends an insignificant amount of time in dbdsqr. However, if the singular vectors are needed, our performance profile in Figure 10 using the reference implementation of pdgesvd reveals that the execution time can be dominated by the time to compute the singular vectors of the bidiagonal matrix. The reason is that pdgesvd has all the MPI processes in the same column or row of the processor grid redundantly compute the left or right singular vectors, respectively, of the bidiagonal matrix, which are distributed to the process group. Compared with pdgesvd, LAPACK's dgesvd obtained higher performance by using dbdsqr with multithreaded BLAS. The reference implementation of pdgesvd obtained about the same performance as that of MKL's pdgesvd when linked to MKL BLAS and LAPACK.

Finally, ScaLAPACK supports only the QR iteration algorithm for computing the SVD of the bidiagonal matrix, using LAPACK's dbdsqr, while, as shown in section 7, the D\&C process in LAPACK's dbdsdc may be faster than dbdsqr.

7. Singular Vectors from the Divide and Conquer Process. For solving the bidiagonal SVD subproblem, QR iteration and the related qd algorithms may take as much as $80 \%$ of the total time when computing singular vectors of a dense matrix [56]. $\mathrm{Gu}$ and Eisenstat introduced the bidiagonal D\&C [57, 59] algorithm, which may be an order of magnitude faster on some machines [56]. The development of D\&C was 
based on prior work focusing on computing eigenvalues and singular values $[4,25,52$, $58,74]$.

The D\&C process includes a matrix partitioning step that introduces two large submatrices. The splitting can occur with either "the middle" row $[4,56]$ or column [57]:

$$
B=\left[\begin{array}{cc}
B_{1} & 0 \\
\alpha_{k} e_{k} & \beta_{k} e_{1} \\
0 & B_{2}
\end{array}\right] \quad \text { or } \quad B=\left[\begin{array}{ccc}
B_{1} & \alpha_{k} e_{k} & 0 \\
0 & \beta_{k} e_{1} & B_{2}
\end{array}\right] .
$$

Note that after the partitioning, $B_{1}$ might not be square, even though $B$ was. The fix is to append a zero row or column [57] to obtain the desired shape.

In either the row or the column case, the process continues recursively to obtain the SVD of $B_{1}$ and $B_{2}$, which can be used to decompose $B$ as

$$
B=Q_{r} M_{r} W_{r} \quad \text { or } \quad B=Q_{c} M_{c} W_{c},
$$

with orthogonal matrices $Q_{r}, W_{r}, Q_{c}$, and $W_{c} . M_{r}$ and $M_{c}$ have a special structure: only the diagonal and either a single row or column, respectively, are nonzero,

$$
M_{r}=\left[\begin{array}{cccc}
z_{1} & z_{2} & \ldots & z_{n} \\
& d_{2} & & \\
& & \ddots & \\
& & & d_{n}
\end{array}\right] \quad \text { or } \quad M_{c}=\left[\begin{array}{cccc}
z_{1} & & & \\
z_{2} & d_{2} & & \\
\vdots & & \ddots & \\
z_{n} & & & d_{n}
\end{array}\right]
$$

Trivially, because matrices $Q_{r}, W_{r}, Q_{c}$, and $W_{c}$ are orthogonal, $B$ and $M_{r}$ or $M_{c}$ share singular values $\sigma_{i}$. A number of theorems and lemmas [74, 57] lead to a fast and numerically (relatively) stable procedure for computing the SVD of $M_{r}$ or $M_{c}$ as $U_{m} \Sigma_{m} V_{m}^{T}$. The interlacing property sets the bounds and ranges for $\sigma_{i}$,

$$
0 \equiv d_{1}<\sigma_{1}<d_{2}<\cdots<d_{n}<\sigma_{n}<d_{n}+\|z\|_{2}
$$

and the secular equation

$$
f(\sigma)=1+\sum_{k=1}^{n} \frac{z_{k}^{2}}{d_{k}^{2}-\sigma^{2}}=0
$$

is used for computing the values $\sigma_{i}$ with a specifically crafted root finder that accounts for floating-point vagaries of past and modern computing systems [80]. The corresponding formulas for the left singular vectors $U_{m}$,

$$
u_{i}=\left[\frac{z_{1}}{d_{1}^{2}-\sigma_{i}^{2}}, \ldots, \frac{z_{n}}{d_{n}^{2}-\sigma_{i}^{2}}\right]^{T} / \sqrt{\sum_{k=1}^{n} \frac{z_{k}^{2}}{\left(d_{k}^{2}-\sigma_{i}^{2}\right)^{2}}},
$$

and the right singular vectors $V_{m}$,

$$
v_{i}=\left[-1, \frac{d_{2} z_{2}}{d_{2}^{2}-\sigma_{i}^{2}}, \ldots, \frac{d_{n} z_{n}}{d_{n}^{2}-\sigma_{i}^{2}}\right]^{T} / \sqrt{1+\sum_{k=2}^{n} \frac{\left(d_{k} z_{k}\right)^{2}}{\left(d_{k}^{2}-\sigma_{i}^{2}\right)^{2}}}
$$

indicate that there could be accuracy problems for the components of either set of vectors, even though the computed singular values $\hat{\sigma}$ are a good approximation of 


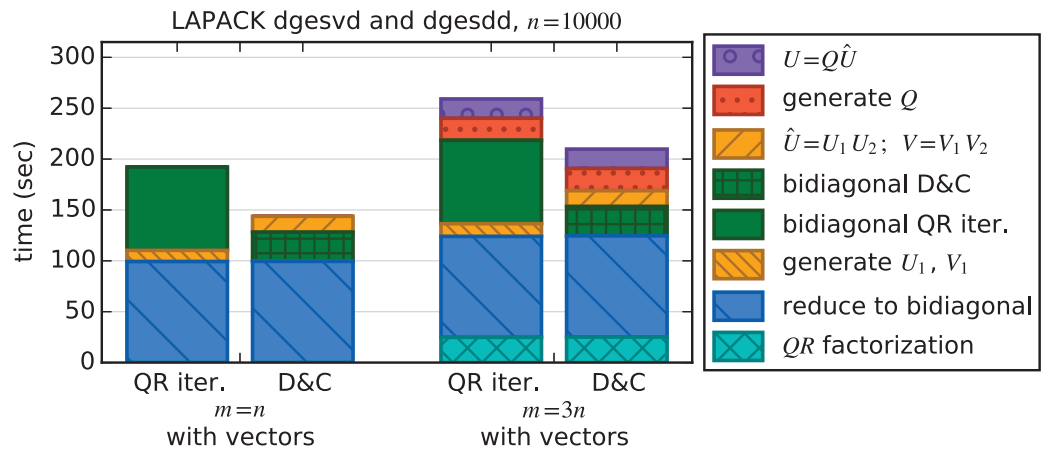

Fig. II Profile comparing LAPACK QR iteration (dgesvd) and DEC (dgesdd) algorithms. For QR iteration, it generates $U_{1}$ and $V_{1}$, then updates them with $U_{2}$ and $V_{2}$ during $Q R$ iteration. $D \mathscr{B} C$ generates $U_{2}$ and $V_{2}$, then multiplies $\hat{U}=U_{1} U_{2}$ and $V=V_{1} V_{2}$ afterwards.

the exact singular values $\sigma$, because the ratios $z_{k} /\left(d_{k}^{2}-\sigma_{i}^{2}\right)$ in (5) and (6) can be inaccurate. The trick is not to use the same $M_{r}$ or $M_{c}$ matrices that were used to compute the approximate singular values $\hat{\sigma}_{i}$, but to instead construct new $\hat{M}_{r}$ or $\hat{M}_{c}$ based on $\hat{\sigma}_{i}$ that improve the accuracy of expressions in (5) and $(6)[80,59]$. This can dramatically diminish the departure from orthogonality for both sets of vectors.

After computing $U_{m}$ and $V_{m}$, the SVD of $B$ is computed by multiplying $Q_{r} U_{m}$ and $V_{m}^{T} W_{r}$. This is done for each $B$ matrix in the recursion tree, from the leaf nodes to the root. Most of the cost of D\&C is in these matrix multiplications, which are Level 3 BLAS. In particular, most of the cost is at the higher levels of the recursion tree, near the root node, as the matrices get larger.

Li et al. [81] recently showed that D\&C internally generates matrices with structure that can be exploited. The matrices $U_{m}$ and $V_{m}$, which are the singular vectors of $M$, have low rank off-diagonal blocks that can be efficiently compressed with hierarchically semiseparable (HSS) matrices. Using HSS improves the speed of matrix multiplies, reducing the cost of the bidiagonal D\&C step from $O\left(n^{3}\right)$ to $O\left(n^{2} r\right)$, where $r$ depends on the matrix but usually $r \ll n$ for large $n$. Li et al. [81] showed over $3 \times$ improvement compared to Intel MKL for the bidiagonal D\&C step on large matrices.

D\&C restructures the SVD algorithm somewhat, as is shown in Algorithm 4, compared with the QR iteration version in Algorithm 2. D\&C directly computes the SVD of the bidiagonal matrix $B=U_{2} \Sigma V_{2}$, and then multiplies $U=U_{1} U_{2}$ and $V=$ $V_{1} V_{2}$ afterwards (using dormbr), while with QR iteration, LAPACK first generates $U_{1}$ and $V_{1}$ (using dorgbr), then accumulates $U_{2}$ and $V_{2}$ onto $U_{1}$ and $V_{1}$ during QR iteration. The profile in Figure 11 shows this difference in the bidiagonal QR iteration (green, no hatching) vs. D\&C steps (green, + hatching); and the generate $U_{1}, V_{1}$ (orange, IIII hatching) vs. $U=U_{1} U_{2}, V=V_{1} V_{2}$ (orange, // hatching) steps. The main advantage of the $\mathrm{D} \& \mathrm{C}$ approach is that it saves nearly half the flops compared to $\mathrm{QR}$ iteration when computing singular vectors. For a square matrix, $\mathrm{D} \& \mathrm{C}$ is $\approx 9 n^{3}$ flops, compared to $\approx 17 n^{3}$ for QR iteration (see Table 1 ). We can observe this as a reduction in time for the steps mentioned in Figure 11.

Figure 12 shows the relative speedup over EISPACK when using a modern multicore system, for both the $\mathrm{D} \& \mathrm{C}$ and $\mathrm{QR}$ iteration algorithms. We see that for square and tall 3:1 matrices, D\&C is consistently faster than QR iteration. Because of the initial QR factorization (described in subsection 5.4) the advantage decreases as $m$ 


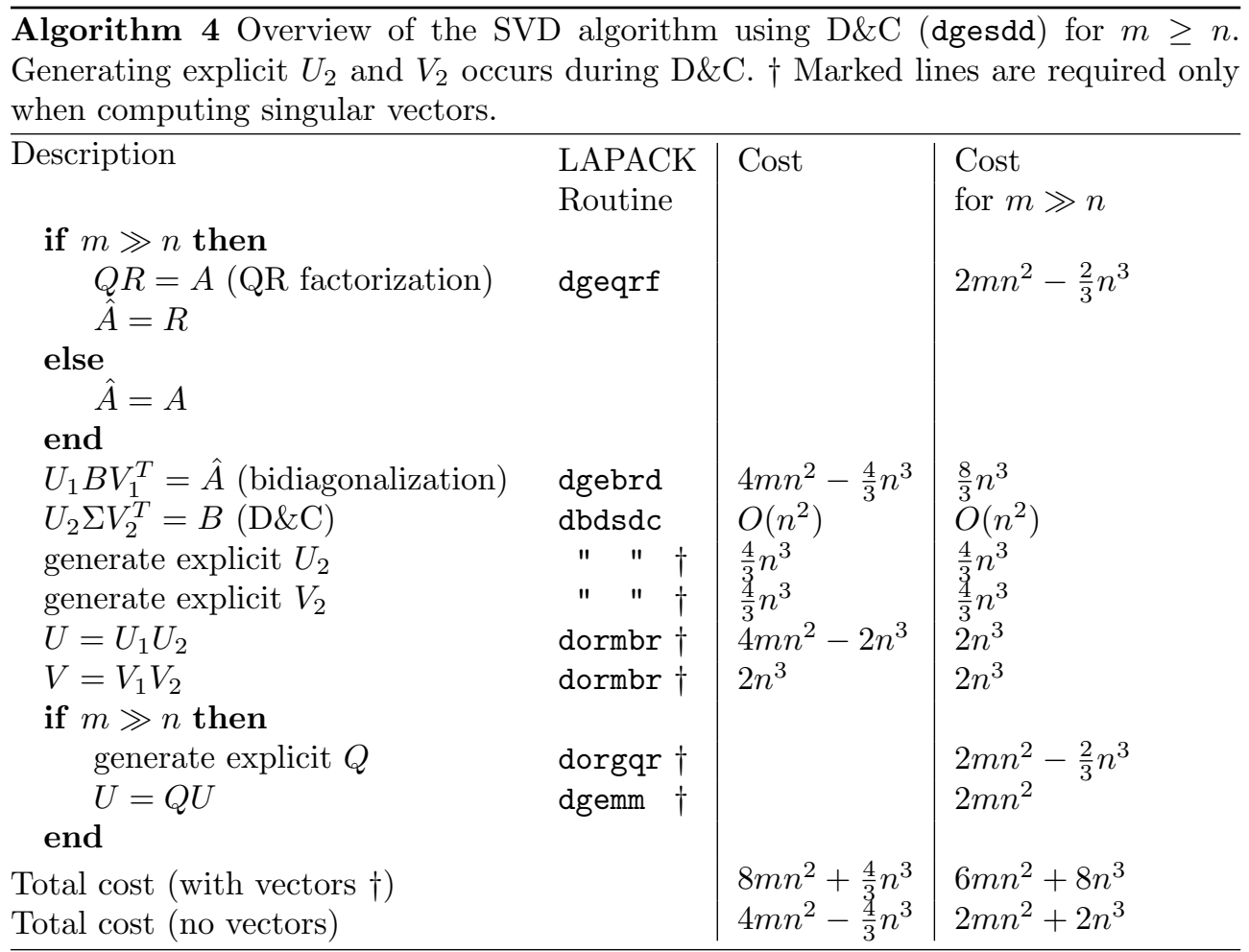

grows relative to $n$, so that for a very tall matrix, both methods take nearly the same time, as is seen by the 1000:1 case in Figure 12(c). It may be safely assumed that $\mathrm{D} \& \mathrm{C}$ is superior to the QR iteration algorithm for most scenarios, and the worst case is when both perform at the same speed. When computing only singular values, not singular vectors, LAPACK always uses QR iteration, since in that case both bidiagonal QR iteration and $\mathrm{D} \& \mathrm{C}$ are $O\left(n^{2}\right)$, while the overall time will be dominated by the $O\left(n^{3}\right)$ reduction to bidiagonal.

8. Bisection and Inverse Iteration. LAPACK 3.6.0 introduced a bisection method (dgesvdx) to compute all or a subset of the singular values and vectors [86]. Similar to QR iteration (dgesvd) and D\&C (dgesdd), it first reduces the matrix $A$ to bidiagonal form $B$. Then it computes the singular values of $B$ based on bisection and the corresponding singular vectors by inverse iteration, using dbdsvdx. For computing the SVD of $B$, it converts the bidiagonal matrix $B$ to the Golub-Kahan [53] symmetric tridiagonal matrix $T$ of dimension $2 n$,

$$
T=\operatorname{tridiag}\left(\begin{array}{cccccccc} 
& b_{1,1} & b_{1,2} & b_{2,2} & b_{2,3} & \ldots & b_{n-1, n} & b_{n, n} \\
0 & 0 & 0 & 0 & 0 & \ldots & 0 & 0 \\
& b_{1,1} & b_{1,2} & b_{2,2} & b_{2,3} & \ldots & b_{n-1, n} & b_{n, n}
\end{array}\right),
$$

whose eigenpairs are $\left( \pm \sigma_{j}, z_{j}\right)$, where $\sigma_{j}$ is the $j$ th singular value of $B$. Elements of $u_{j}$ and $v_{j}$, the corresponding left and right singular vectors of $B$, are interleaved in the eigenvector as $z_{j}=\left[v_{1, j},-u_{1, j}, v_{2, j},-u_{2, j}, \ldots, v_{n, j},-u_{n, j}\right] / \sqrt{2}$. Instead of developing new subroutines, dbdsvdx relies on the subroutines dstebz and dstein that compute the eigenvalues and eigenvectors, respectively, of the symmetric tridiagonal matrix based on bisection and inverse iteration. 


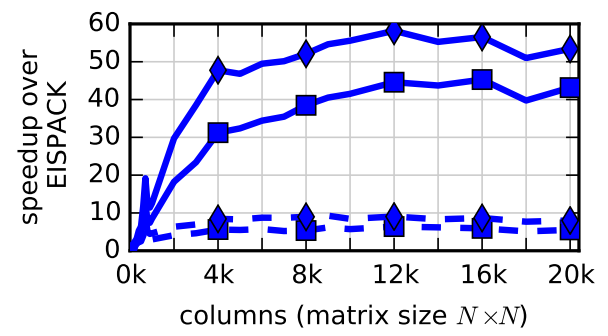

(a) square, with vectors

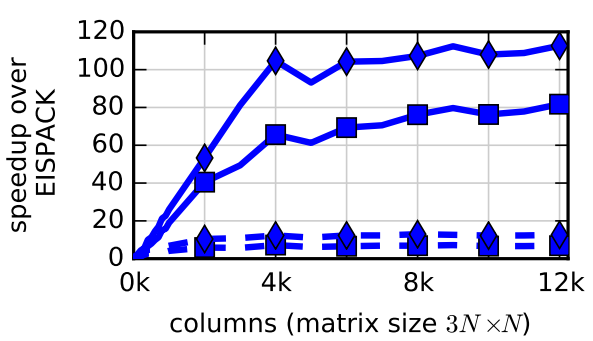

(b) tall, $3: 1$, with vectors

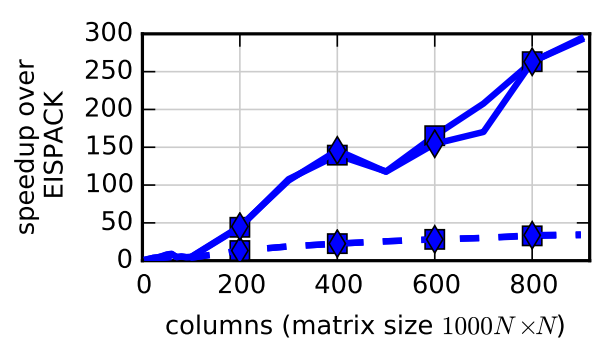

(c) tall, 1000:1, with vectors

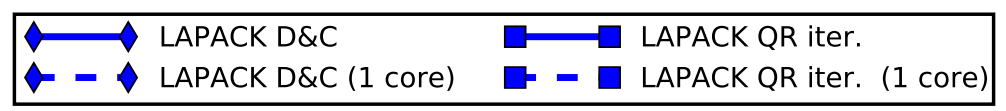

Fig. 12 Comparison of LAPACK DEC to QR iteration. Solid lines represent 16-core runs; dashed lines represent single-core runs.

The bisection algorithm implemented in dstebz uses Sylvester's inertia theorem to compute the number of eigenvalues within a certain interval. In particular, the algorithm relies on the $L D L^{T}$ factorization of the matrix $T$, where $L$ is a lowertriangular matrix with unit diagonal and $D$ is a diagonal matrix. For the symmetric tridiagonal matrix $T$, the diagonal matrix $D$ can be computed with $O(n)$ flops based on the simple recurrence formula

$$
d_{i, i}=\left(t_{i, i}-s\right)-\frac{t_{i-1, i}^{2}}{d_{i-1, i-1}} .
$$

Given the $L D L^{T}$ factorization of the matrix $T-s I$ for a certain shift value $s$, the number of negative elements of $D$ is equal to the number of eigenvalues of $T$ smaller than $s$. In other words, given the $L D L^{T}$ factorizations of two shifted matrices, $T-s_{1} I$ and $T-s_{2} I$, with $s_{1}<s_{2}$, if there are $n_{1}$ and $n_{2}$ negative entries in their respective diagonal matrices, then there are $n_{2}-n_{1}$ eigenvalues in the interval $\left(s_{1}, s_{2}\right]$. In addition, for the tridiagonal matrix, it can be shown that the $L D L^{T}$ factorization without pivoting can be reliably used for counting the number of eigenvalues [34, 75]. Based on these observations, dstebz keeps bisecting the initial interval containing all the desired eigenvalues until it finds a small enough interval for each eigenvalue such that the computed eigenvalue has the desired accuracy. Each bisection improves the accuracy of the eigenvalue by one bit, hence the iteration converges linearly. An advantage of bisection is that it can be naturally adapted to compute a subset of eigenvalues, which was one of the motivations for introducing dgesvdx [86]. 
Given the eigenvalues computed by dstebz, dstein computes the corresponding eigenvectors based on inverse iteration. Namely, for each computed eigenvalue $\lambda$, it first computes the $\mathrm{LU}$ factorization of the shifted matrix $A-\lambda I$ with partial pivoting. Then the corresponding eigenvector of $\lambda$ is computed by inverse iteration, with a starting vector whose entries are random numbers uniformly distributed in the interval $(-1,1)$. Given an accurate eigenvalue approximation, inverse iteration converges quickly [72] (e.g., dstein sets the maximum number of iterations to be five). However, when the eigenvalues are close to each other, inverse iteration may fail to generate orthogonal eigenvectors. To recover the orthogonality among such vectors, dstein reorthogonalizes the vectors based on the modified Gram-Schmidt procedure. Unfortunately, when the computed eigenvectors are nearly dependent, the eigenvectors may not be accurate after the reorthogonalization [33]. In addition, if many of the eigenvalues are close to each other, this reorthogonalization cost could become significant with $O\left(k^{2} n\right)$ flops for computing $k$ eigenvectors in the worst case. As a result, in our experiments shown in Figure 13, we saw that when computing all the singular values and vectors, bisection can be significantly slower than other methods. Even with 16 threads available, it is slower than the single-threaded QR iteration (dashed line). Bisection and inverse iteration are embarrassingly parallel - each eigenvalue and eigenvector may be computed independently - however, LAPACK does not currently include such explicit parallelization, instead relying primarily on parallelism within the BLAS, which is not advantageous in this case. On the other hand, as seen in Figure 14, when only a subset of $k$ singular values and vectors is computed, we observed that bisection and inverse iteration (nonhatched bars) can be up to $2.4 \times$ faster than D\&C (black bar and dashed line), which must compute all the singular values and vectors. Depending on the matrix type, when computing $k=400$ or $k=600$ vectors out of $n=3000$, it becomes faster to simply compute all the vectors using D\&C. The exception here is the cluster, with one singular $\sigma_{1}=1$ and all other $\sigma_{i}=1 / \kappa$. In that case, computing any $k>1$ vectors was as slow as computing all vectors with bisection. See section 2 for a description of the matrices.

9. Multiple Relatively Robust Representations (MRRR). MRRR [35, 36] was developed to improve both the performance and the accuracy of inverse iteration. Analysis has shown that MRRR can compute the numerically orthogonal eigenvectors of a symmetric tridiagonal matrix in $O\left(n^{2}\right)$ flops. At the time of preparing this paper, there was no publicly available software package that implements MRRR for computing the SVD of a general matrix, but there were at least two software packages that compute the eigenvalues and eigenvectors of a symmetric tridiagonal matrix using MRRR: dstemr of LAPACK [38] and dstexr due to Willems and Lang [118], which is tailored toward the tridiagonal matrix with zeros on the diagonal, as used in (7) for the SVD. For our experiments, we replaced the symmetric tridiagonal solver (dstevx) used in dgesvdx with dstexr. Performance with dstemr was generally similar but somewhat slower.

One of the main drawbacks of inverse iteration is that, for the eigenvalues with small relative gaps, the computed eigenvectors may not be orthogonal to each other. Hence, reorthogonalization is needed. This increases the computational cost and potentially leads to loss of accuracy in the computed eigenvectors. To address these issues, MRRR combines several techniques.

First, though the eigenvectors are invariant under a diagonal shift, we can increase their relative gaps by diagonally shifting the matrix. For instance, let us define the relative gap between two eigenvalues $\lambda_{i}$ and $\lambda_{j}$ to be $\frac{\left|\lambda_{i}-\lambda_{j}\right|}{\max \left(\left|\lambda_{i}\right|,\left|\lambda_{j}\right|\right)}$. Then, we can 


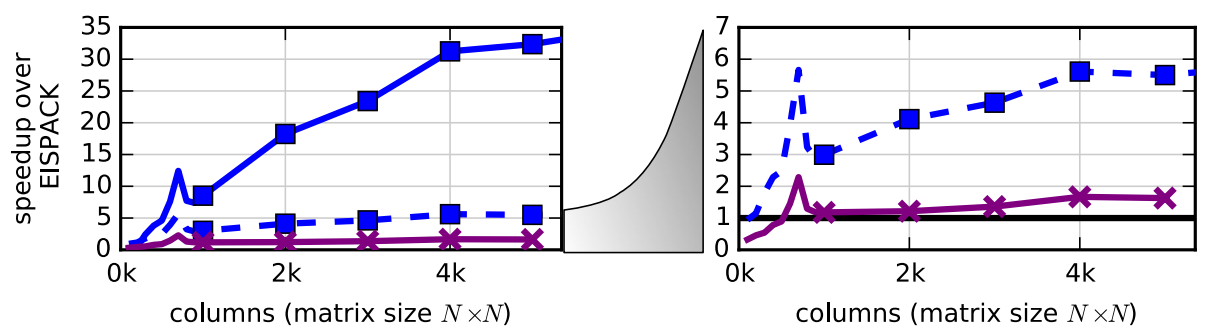

(a) square, with vectors (right graph is zoom of left graph)

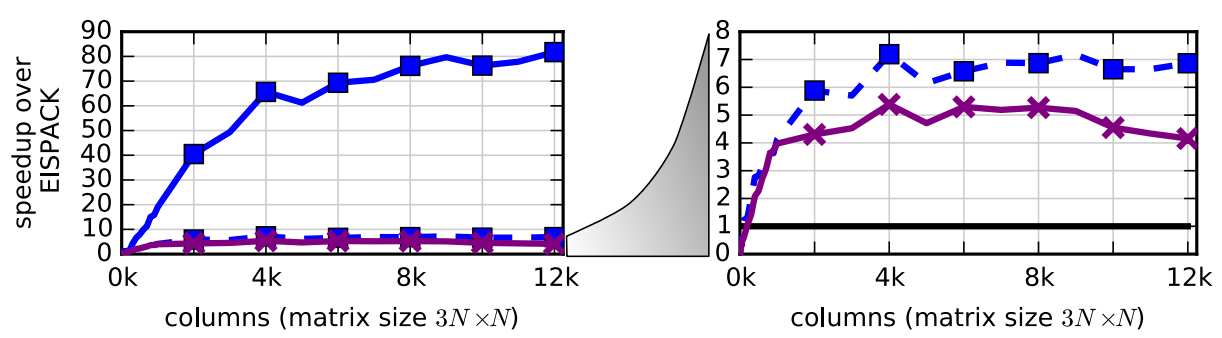

(b) tall, 3:1, with vectors (right graph is zoom of left graph)

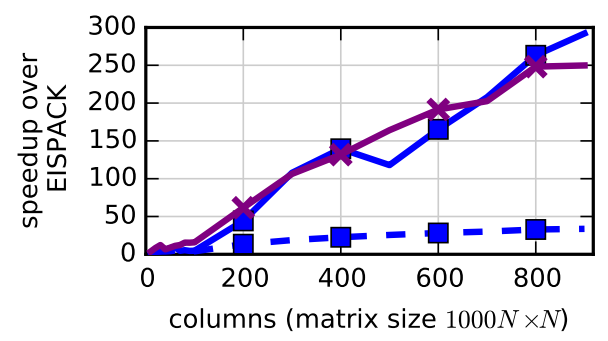

(c) tall, 1000:1, with vectors

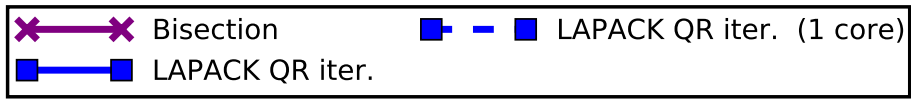

Fig. 13 Comparison of bisection with QR iteration. Solid lines represent 16 -core runs; dashed lines represent single-core runs.

increase their relative gap by a factor of $\frac{|\lambda|}{|\lambda-\tau|}$ when we diagonally shift the matrix using a shift $\tau$ that is close to $\lambda$.

Hence, before applying inverse iteration, MRRR recursively refines the approximation to the eigenvalues and applies appropriate diagonal shifts to a cluster of eigenvalues such that it can guarantee large enough relative gaps between all the eigenvalues of $T$ to maintain the orthogonality among the computed eigenvectors without reorthogonalization. For instance, given two approximate eigenvalues $\lambda_{i}$ and $\lambda_{j}$, inverse iteration is used to compute their respective eigenvectors $v_{i}$ and $v_{j}$ with small residual norms, i.e.,

$$
\left|T v_{k}-\lambda v_{k}\right|=O(n \epsilon|T|) \text { for } k=i \text { and } j .
$$




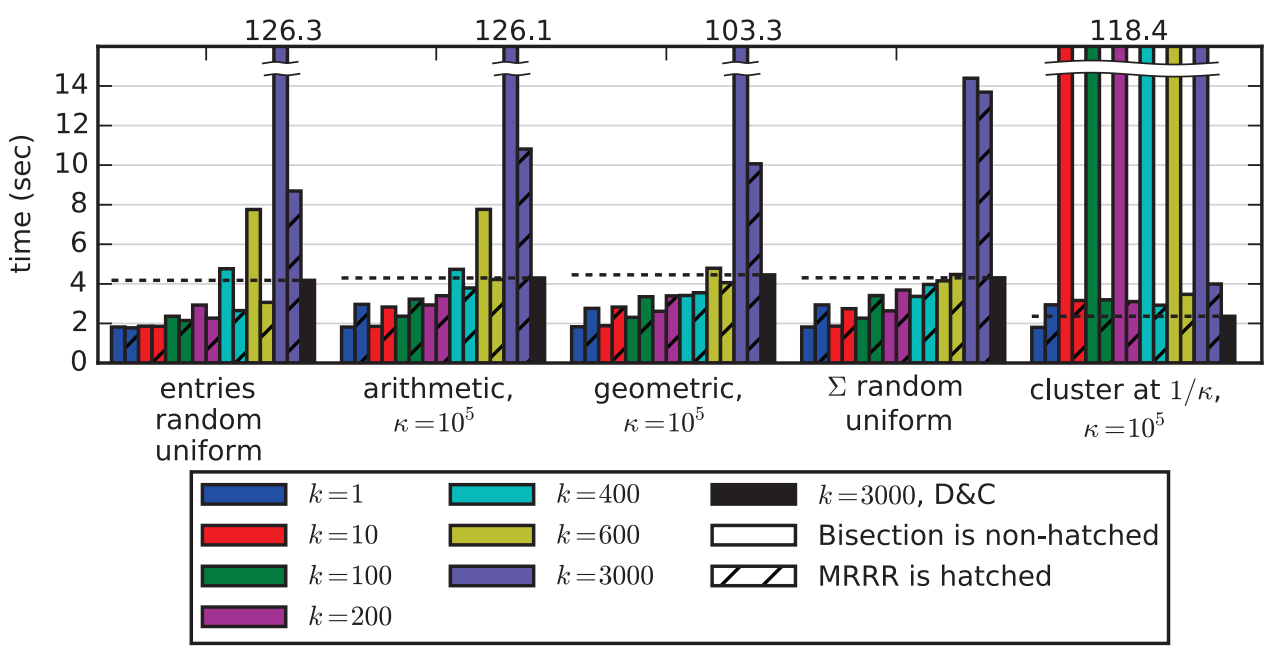

Fig. 14 Results for computing $k$ singular vectors of an $n \times n$ matrix, $n=3000$. Dashed lines show $D \& C$ performance for computing all vectors. The numbers on top show the time for bars that exceed the graph's height.

Then, according to $[37,38]$, a realistic bound on their orthogonality error is given by

$$
\left|v_{i}^{T} v_{j}\right|=O\left(\frac{n \epsilon\left(\left|\lambda_{i}\right|+\left|\lambda_{j}\right|\right)}{\left|\lambda_{i}-\lambda_{j}\right|}\right) .
$$

Therefore, if the gap $\left|\lambda_{i}-\lambda_{j}\right|$ is of the same order as the eigenvalues, their eigenvectors are numerically orthogonal, i.e., $\left|v_{i}^{T} v_{j}\right|=O(n \epsilon)$.

There are several parameters that can be tuned to improve the performance [38], including the accuracy of the eigenvalue approximation computed at each step and the choice of the algorithm for computing the approximation (e.g., bisection, QR iteration, or Rayleigh quotient correction).

Second, while computing the eigenvalues (e.g., applying the diagonal shift), a small relative roundoff error in the entry of the tridiagonal matrix could result in a large relative error in the computed eigenvalues, especially in those with small magnitudes. To preserve the relatively high accuracy of the computed eigenvalues, MRRR stores the intermediate matrices in particular representations, referred to as the MRRR of the matrices. For instance, it has been shown that the $L D L^{T}$ representation of the tridiagonal matrix $T$, without pivoting, is relatively robust, even with the presence of the element growth [31]. Hence, MRRR stores the sequence of intermediate matrices with different shifts in their $L D L^{T}$ forms.

Third, for an eigenvalue with a small relative gap, the cost of inverse iteration may be high, requiring a few iterations to obtain the eigenvector with a small relative residual norm. Fortunately, there is at least one starting vector with which inverse iteration converges in one iteration. For example, when the $i$ th column of $(T-\lambda I)^{-1}$ has the largest column norm, then with the canonical vector $e_{i}$ as the starting vector, one step of inverse iteration computes the approximate eigenvector $x$ such that

$$
|T x-\lambda x| \leq \sqrt{n}|\lambda-\bar{\lambda}|
$$

where $\bar{\lambda}$ is the exact eigenvalue [72]. Hence, if the eigenvalue is computed to a high 


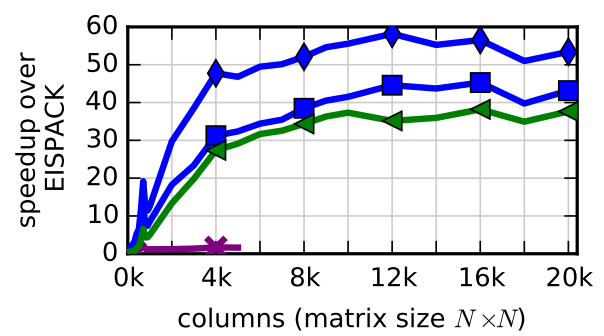

(a) square, with vectors

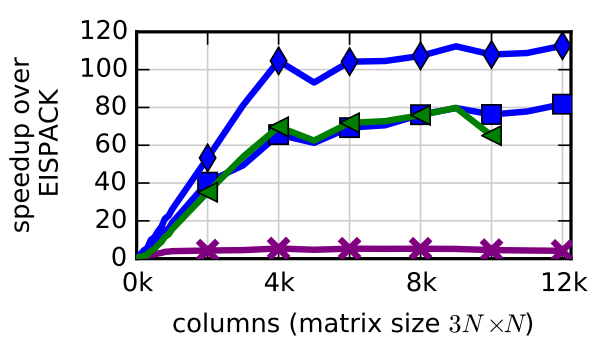

(b) tall, $3: 1$, with vectors

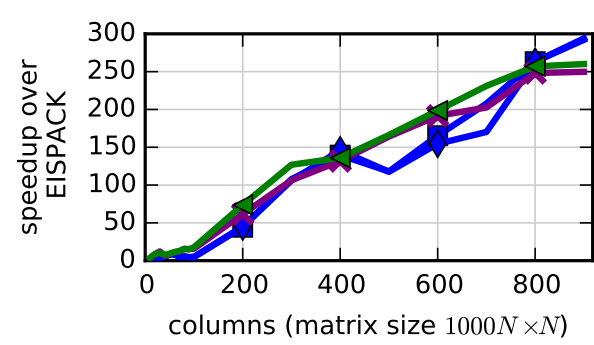

(c) tall, 1000:1, with vectors

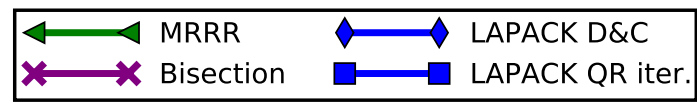

Fig. I5 Comparison of $M R R R$ with $Q R$ iteration, $D E C$, and bisection.

relative accuracy,

$$
|\lambda-\bar{\lambda}|=O(\epsilon|\bar{\lambda}|)
$$

(e.g., using bisection with $O(n)$ flops), then the computed eigenpair obtains a small relative residual norm,

$$
|T x-\lambda x|=O(n \epsilon|\bar{\lambda}|) .
$$

There is an algorithm to find the column of $(T-\lambda I)^{-1}$ with largest norm with $O(n)$ flops [98]. In addition, if a twisted factorization is used to find the starting vector, then it can be shown that the computed eigenpairs have small residual norm with respect to the original matrix $T[36,37]$. The twisted factorization must be carefully computed for $T$ with a zero diagonal because the leading dimension of an odd dimension is singular. To enhance the numerical stability, dstexr computes a block variant of the factorization [118].

As can be seen in Figure 15, by avoiding the reorthogonalization, MRRR can significantly improve the performance of inverse iteration, making MRRR comparable to $\mathrm{QR}$ iteration. However, D\&C is often faster.

Especially for large matrices, we noticed numerical issues where the backward error $\left\|A-U \Sigma V^{T}\right\| /(\min (m, n)\|A\|)$ was large, e.g., $10^{-4}$ instead of $10^{-16}$ as expected. Further tests in section 13 show that, even when the above error is acceptable, MRRR has poor relative error for the singular values. Marques and Vasconcelos [86] also observed numerical issues with the existing MRRR implementation.

When only a subset of $k$ singular vectors are computed, we observe in Figure 14 that inverse iteration can be up to $1.6 \times$ faster than MRRR for a small number of 
vectors $(k=1$ or 10$)$. For a larger subset of $k=600$ vectors, MRRR can be up to $1.8 \times$ faster than bisection, but in this case, only for the random entries matrix is MRRR significantly faster $(1.3 \times)$ than computing all the singular vectors with D\&C. The exception is the cluster matrix, where for $k>1$, MRRR is $30 \times$ faster than bisection, but always slower than using D\&C.

10. MAGMA Implementation for Accelerator Architectures. Accelerators such as GPUs and the Intel Xeon Phi provide a high degree of parallelism and a larger memory bandwidth than traditional multicore CPUs. The MAGMA library was developed to address this new architecture and accelerates most phases of the SVD algorithm: reduction to bidiagonal, bidiagonal D\&C, and computation of singular vectors. For tall-skinny matrices, it also accelerates the initial QR factorization and generating $Q$.

The most prominent place to start is an accelerated version of the bidiagonal reduction [109]. We have seen in Figures 6 and 11 that this phase (blue tier with $\backslash \backslash$ hatching) takes from $50 \%$ to $70 \%$ of the time for a square matrix when computing singular vectors, and $98 \%$ of the time when computing only singular values (no vectors). As described in section 5, the bidiagonal reduction has half its flops in Level 2 BLAS and half in Level 3 BLAS. Accelerators are known for achieving very high performance on compute-intensive, Level 3 BLAS operations. On an NVIDIA K40c GPU, cuBLAS achieves 1245 Gflop/s with dgemm, compared with 315 Gflop/s using Intel MKL on the multicore CPU. Due to the accelerator's large memory bandwidth, the memorybound Level 2 BLAS operations are also significantly faster, achieving $45 \mathrm{Gflop} / \mathrm{s}$ with cuBLAS dgemv, compared with 14 Gflop/s on the multicore CPU. Therefore, both the trailing matrix-vector product (dgemv) and the trailing matrix update (dgemm) are performed on the accelerator. The small panel operations - constructing Householder reflectors - are performed on the CPU, which is better at serial operations with more control flow. This incurs CPU-to-GPU communication of a couple of vectors for each dgemv operation during the panel. Due to dependencies, the trailing matrix update cannot be overlapped with the next panel, as would occur in a one-sided QR factorization. Using the accelerator improves the speed of the bidiagonal reduction by about a factor of 2, as is shown by the profile in Figure 16 (blue tier with $\backslash \backslash$ hatching) and by the square, no vectors case in Figure 17(a), which is dominated by the bidiagonal reduction.

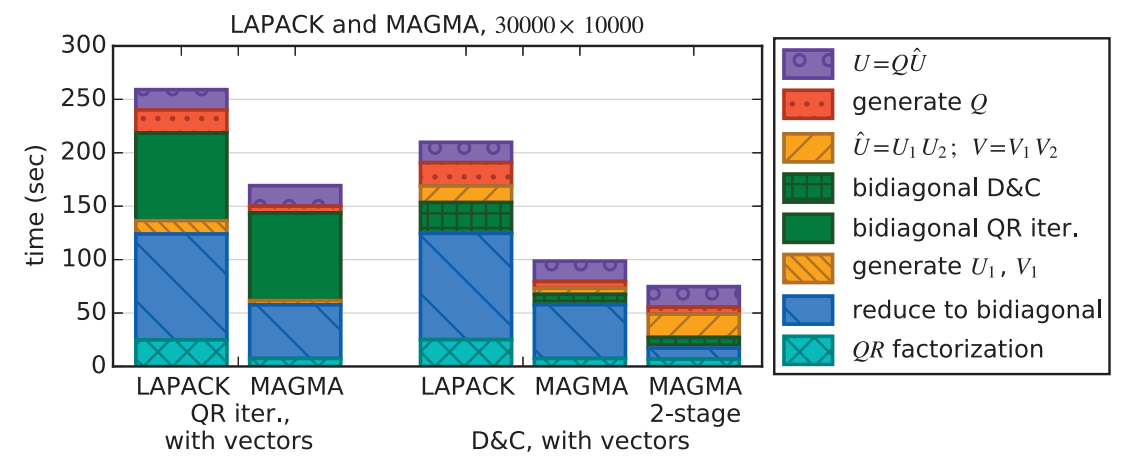

Fig. 16 Profile comparing LAPACK and MAGMA. Most phases are accelerated using the GPU, except the bidiagonal $Q R$ iteration and multiplying $U=Q \hat{U}$. MAGMA 2-stage is described in section 11. 


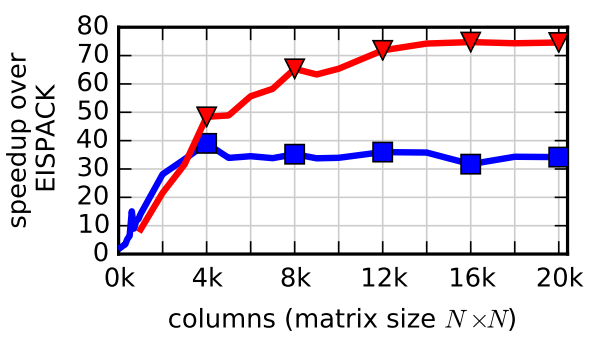

(a) square, no vectors

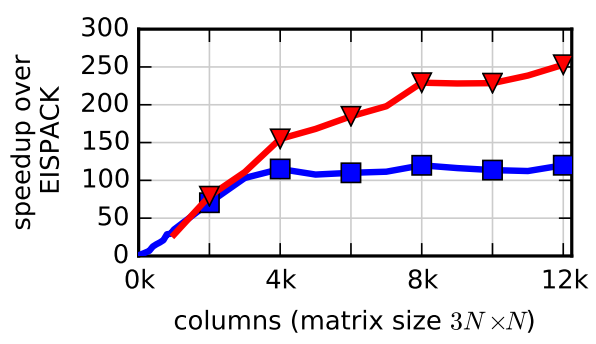

(c) tall, 3:1, no vectors

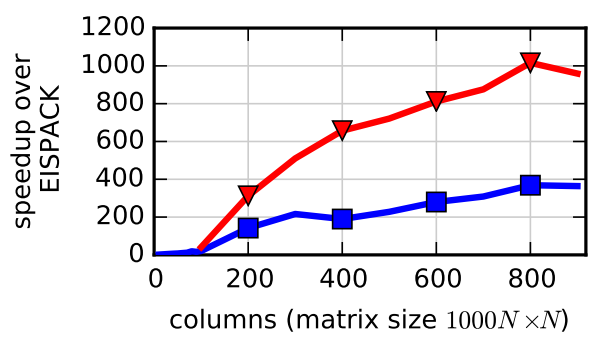

(e) tall, 1000:1, no vectors

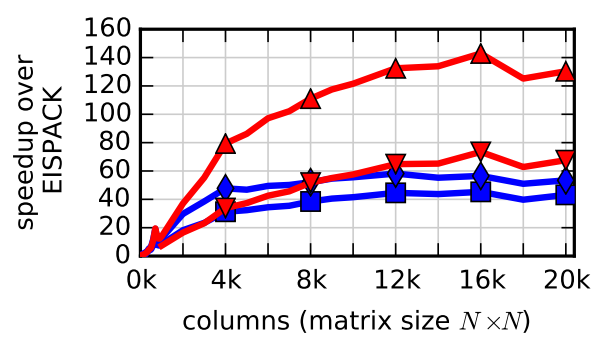

(b) square, with vectors

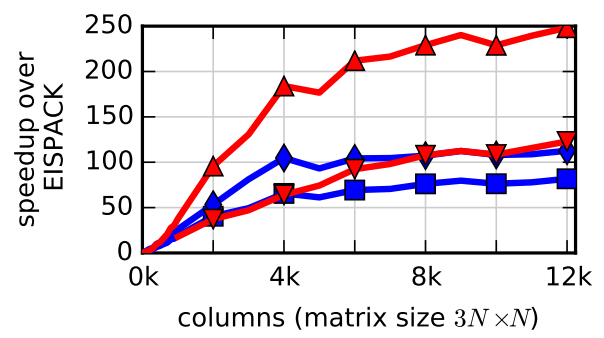

(d) tall, 3:1, with vectors

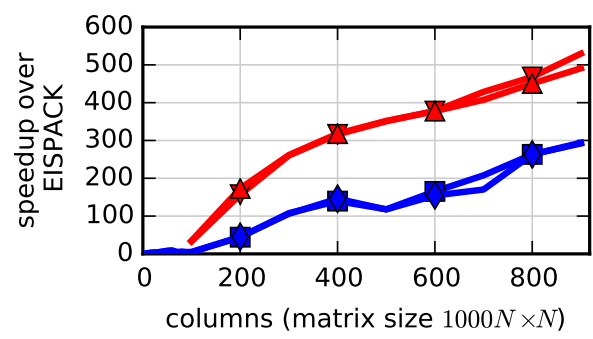

(f) tall, 1000:1, with vectors

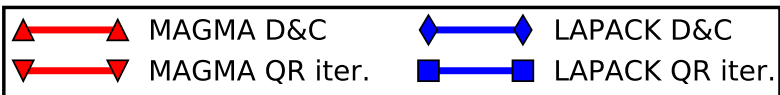

Fig. 17 Comparison of MAGMA with LAPACK.

For the bidiagonal SVD, because D\&C is faster than QR iteration, MAGMA will inherently achieve a better overall speedup using D\&C. We further implement an accelerated version of D\&C [51]. Since most of the operations in D\&C are in multiplying $Q_{r} U_{m}$ and $V_{m}^{T} W_{r}$ to generate singular vectors, these Level 3 BLAS dgemm operations are assigned to the accelerator. The solution of the secular equation to find the singular values of $M_{c}$ is left on the CPU, since it is a complex iterative algorithm with limited parallelism, as is computing the singular vectors $U_{m}$ and $V_{m}$ of $M_{c}$. These are parallelized on the CPU using OpenMP. MAGMA achieves about a $3 \times$ speedup for the $\mathrm{D} \& \mathrm{C}$ phase compared to LAPACK.

For a tall-skinny $(m \gg n)$ matrix, we accelerate the initial QR factorization [110]. This is a one-sided factorization, so it doesn't have the extra dependencies imposed by the two-sided reduction to bidiagonal form. Panel operations are within a simple block 
column that doesn't involve the trailing matrix. The panel factorization is performed on the CPU, while the trailing matrix update is performed on the accelerator. The accelerator updates the next panel first and sends it back to the CPU so the CPU can start factoring it while the accelerator proceeds with the rest of the trailing matrix update. This overlap allows the factorization to achieve a substantial portion of the peak dgemm speed, up to $970 \mathrm{Gflop} / \mathrm{s}$ with an NVIDIA K40c. The QR factorization phase was up to $3.6 \times$ faster than on the multicore CPU, as is seen in Figure 16 (cyan tier with $\times$ hatching).

There are three routines that solely apply block Householder reflectors, which are implemented as a series of Level 3 BLAS matrix multiplies entirely on the accelerator: (1) for QR iteration, generating explicit $U_{1}$ and $V_{1}$ matrices (dorgbr), (2) for D\&C, multiplying $U_{1} U_{2}$ and $V_{1} V_{2}$ (dormbr), and (3) for a tall-skinny matrix, generating an explicit $Q$ matrix (dorgqr). These were all up to $3.3 \times$ faster when using the accelerator than when using the multicore CPU. We see in Figure 16 that the time for all three of these phases is substantially reduced.

Overall, MAGMA achieves significant improvements using an accelerator for the SVD problem. Figure 17 shows that it is about $2 \times$ faster than LAPACK in most cases. For the square, vectors case in Figure 17(b), MAGMA's SVD using D\&C is $2.5 \times$ LAPACK's D\&C version, and $2 \times$ MAGMA's SVD using QR iteration, while MAGMA's SVD using QR iteration is only 1.6× LAPACK's QR iteration version, due to both D\&C being inherently faster and having an accelerated version of the D\&C phase. In the tall 1000:1 case in Figure 17(e), MAGMA is 2.6× faster, and for some sizes as much as $3.5 \times$ faster, than LAPACK, and up to $1000 \times$ faster than EISPACK, due to the accelerated QR factorization.

II. Two-Stage Reduction. While all the preceding algorithmic and architectural improvements have greatly increased the speed of the SVD, all these one-stage methods remain limited by the memory-bound, Level 2 BLAS operations. To overcome the limitations of the one-stage approach, Großer and Lang $[78,55]$ introduced the two-stage bidiagonal reduction, which increases the use of compute-intensive Level 3 BLAS operations. The idea behind the two-stage algorithm is to split the original one-stage bidiagonal reduction into a compute-intensive phase (first stage) and a memory-bound phase (second or bulge-chasing stage), as represented in Figure 18. The first stage reduces the original general dense matrix to a band form (either upper or lower), and the second stage reduces the band form to bidiagonal form (again, either upper or lower). The algorithm maps computational tasks to the strengths of the available hardware components, taking care of the data reuse. It also uses techniques to mix between dynamic and static scheduling to extract efficiency and performance. We implemented two-stage algorithms in the PLASMA library for multicore environments $[82,83,62,60]$, the DPLASMA library for distributed environments $[19,18]$, and the MAGMA library for accelerator architectures [51]. Similar two-stage reduction [61] and multistage successive band reduction (SBR) $[13,6]$ to tridiagonal have been used for the symmetric eigenvalue problem. A multistage approach would also work for the bidiagonal reduction, and could be advantageous for achieving optimal communication costs at each stage. However, when computing singular vectors, each stage adds cost to the back transformation, making a multistage approach less favorable.

II.I. First Stage: Compute-Intensive and Efficient Kernels. The first stage applies a sequence of blocked Householder transformations to reduce the general dense matrix to an upper (for $m \geq n$ ) band matrix. This stage uses compute-intensive 


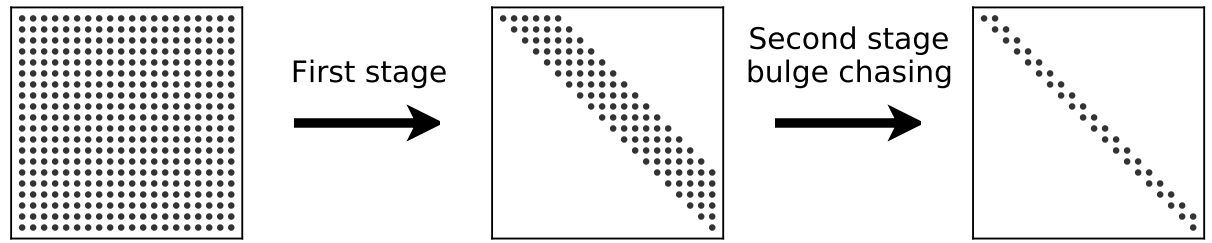

Fig. 18 Two-stage technique for the reduction phase.

matrix-multiply kernels that eliminate the memory-bound matrix-vector products from the one-stage panel factorization.

The first stage proceeds by computing a QR factorization of a block column to annihilate entries below the diagonal and updating the trailing matrix, as shown in Figure 19. It then computes an LQ factorization of a block row to annihilate entries right of the upper bandwidth, and updates the trailing matrix. It repeats the factoring of block columns and block rows, until the entire matrix is brought to band form. The width of the block columns and rows is the resulting matrix bandwidth, $n_{b}$.

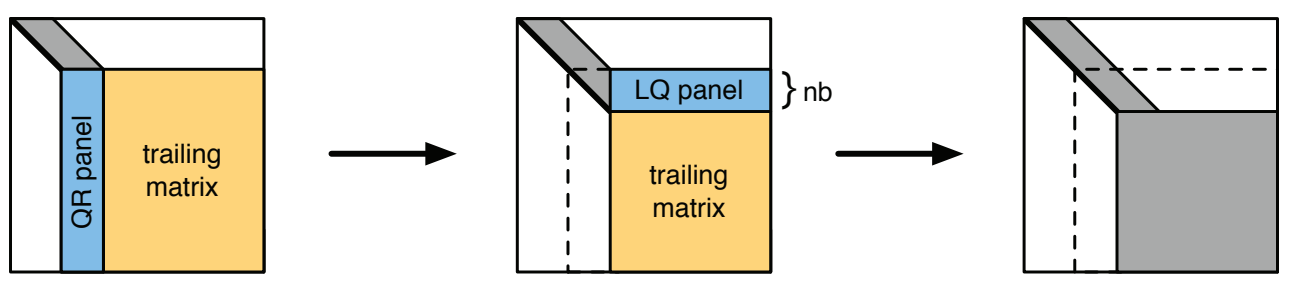

Fig. 19 One panel of the first-stage reduction to band form.

The PLASMA and DPLASMA implementations use a tile algorithm [1] that makes them highly parallel. The matrix is split into tiles of size $n_{b} \times n_{b}$, where $n_{b}$ is the matrix bandwidth. Data within each tile is stored contiguously in memory. A panel factorization is a series of $\mathrm{QR}$ or LQ factorizations done between pairs of tiles; once a pair of tiles has been factored, updates on the corresponding portions of the trailing matrix can start immediately, before the rest of the panel has finished factoring. This unlocks a large amount of parallelism very quickly. The algorithm then proceeds as a collection of interdependent tasks that operate on the tile data layout and are scheduled in an out-of-order fashion using either the OpenMP runtime for PLASMA or the powerful PaRSEC distributed runtime system for DPLASMA.

The MAGMA implementation uses a standard column-wise layout. It does the QR and LQ factorizations on the CPU, copies the block Householder reflectors to the accelerator, and updates the trailing matrix on the accelerator. Unlike in the onesided factorizations, it cannot start the next panel until the trailing matrix update is finished due to data dependencies.

The first stage's cost is $\frac{8}{3} n^{3}$ operations in Level 3 BLAS. As shown in [60], the performance of this stage is comparable to the performance of the QR factorization and can reach a high percentage of the machine's peak.

II.2. Second Stage: Cache-Friendly Computational Kernels. The second stage reduces the band form to the final bidiagonal form using a bulge-chasing tech- 


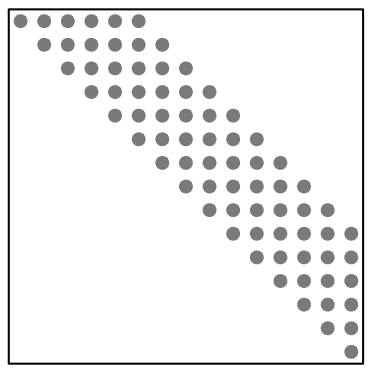

(a) initial band matrix

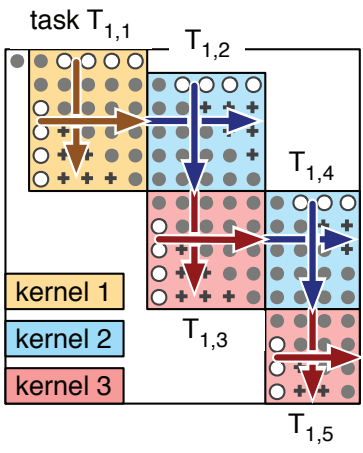

(b) tasks in sweep 1

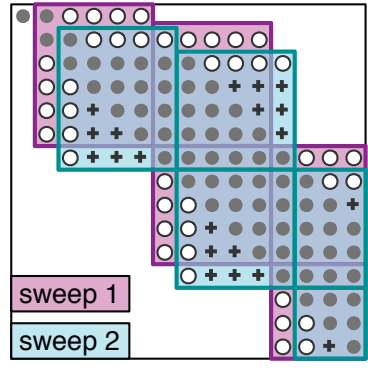

(c) overlap of sweeps

Fig. 20 Bulge-chasing algorithm. "o" indicates eliminated elements; "+" indicates fill. Arrows show application of a Householder reflector on the left $(\rightarrow)$, which updates a block row, and on the right $(\downarrow)$, which updates a block column.

nique. It involves $6 n_{b} n^{2}$ operations, so it takes a small percentage of the total operations, which decreases with $n$. The operations are memory bound, but are fused together as Level 2.5 BLAS [69] for cache efficiency. We designed the algorithm to use fine-grained, memory-aware tasks in an out-of-order, data-flow task-scheduling technique that enhances data locality [60, 61].

The second stage proceeds in a series of sweeps, each sweep bringing one row to bidiagonal and chasing the created fill-in elements down to the bottom right of the matrix using successive orthogonal transformations. It uses three kernels. Kernel 1 (yellow task $T_{1,1}$ in Figure 20(b)) applies a Householder reflector from the right (indicated by the down arrow) to eliminate a row right of the superdiagonal, which also creates a bulge of fill-in beneath the diagonal. It then applies a Householder reflector from the left (indicated by the right arrow) to eliminate the first column of the bulge below the diagonal, and applies the update to the first block column only. The remainder of the bulge is not eliminated, but is instead left for subsequent sweeps to eliminate, as they would reintroduce the same nonzeros.

Kernel 2 (blue task $T_{1,2}$ ) continues to apply the left Householder reflector from kernel 1 (or kernel 3) to the next block column, creating a bulge above the upper bandwidth. It then applies a right Householder reflector to eliminate the first row of the bulge right of the upper bandwidth, updating only the first block row.

Kernel 3 (red task $T_{1,3}$ ) continues to apply the right Householder reflector from kernel 2, creating a bulge below the main diagonal. As in kernel 1, it then applies a left Householder reflector to eliminate the first column of the bulge below the diagonal and updates just the current block column. After kernel 3, kernel 2 is called again (blue task $T_{1,4}$ ) to continue application of the left Householder reflector in the next block column. A sweep consists of calling kernel 1 to bring a row to bidiagonal, followed by repeated calls to kernels 2 and 3 to eliminate the first column or row of the resulting bulges, until the bulges are chased off the bottom-right of the matrix.

For parallelism, once a sweep has finished the first kernel 3, a new sweep can start in parallel. This new sweep is shifted over one column and down one row, as shown in Figure 20(c). Before task $i$ in sweep $s$, denoted as $T_{s, i}$, can start, it depends on task $T_{s-1, i+3}$ in the previous sweep being finished, to ensure that kernels do not update the same entries simultaneously. To maximize cache reuse, tasks are assigned to cores 
based on their data location. Ideally, the band matrix fits into the cores' combined caches, and each sweep cycles through the cores as it progresses down the band.

I I.3. Singular Vectors Computation. The singular vectors of $A$ are computed from the orthogonal transformations used in the reduction to bidiagonal form and from the singular vectors of the bidiagonal form. Recall that for the classical onestage approach, $A=U_{1} B V_{1}^{T}$ and $B=U_{2} \Sigma V_{2}^{T}$. After using D\&C to obtain $U_{2}$ and $V_{2}$, we multiply $U=U_{1} U_{2}$ and $V=V_{1} V_{2}$, costing $2 n^{3}$ each for $U$ and $V$ (if $m=n$ ).

In the case of the two-stage approach, the first stage reduces the original matrix $A$ to a band matrix by applying a two-sided transformation to $A$ such that $A=$ $U_{a} A_{\text {band }} V_{a}^{T}$. Similarly, the second, bulge-chasing stage reduces the band matrix $A_{\text {band }}$ to bidiagonal form by applying a two-sided transformation such that $A_{\text {band }}=U_{b} B V_{b}^{T}$. As a consequence, the singular vectors must be multiplied according to

$$
U=U_{a} U_{b} U_{2} \quad \text { and } \quad V=V_{a} V_{b} V_{2} .
$$

Hence, the two-stage approach introduces a nontrivial amount of extra computationthe application of $U_{b}$ and $V_{b}$-when the singular vectors are needed. The total cost of updating the singular vectors when using the two-stage technique is $2\left(1+\frac{i_{b}}{n_{b}}\right) n^{3}+2 n^{3}$ each for $U$ and $V$, where $n_{b}$ is the bandwidth and $i_{b}$ is an internal blocking; usually $i_{b} \leq n_{b} / 4$. This extra cost compared with the one-stage approach reduces the potential speedup, but as it is in Level 3 BLAS, it does not completely negate the large speedup that we gain by the two-stage bidiagonal reduction.

I I.4. PLASMA Implementation for Multicore. The experiments shown in Figure 21 illustrate the superior efficiency of our two-stage SVD solver compared with the optimized LAPACK version from Intel MKL. Figure 21(a) shows that the bidiagonal reduction itself is $6 \times$ faster than LAPACK, both using 16 cores, and $2.5 \times$ faster than the MAGMA one-stage version. The reason is that LAPACK and MAGMA are bound by the Level 2 BLAS performance, while our two-stage algorithm relies on Level 3 BLAS for most of its computation. When computing singular vectors in Figure 21(b), it is still about $1.8 \times$ faster than LAPACK, even though it requires an extra $2 \times 2\left(1+\frac{i_{b}}{n_{b}}\right) n^{3}$ operations to multiply by $U_{b}$ and $V_{b}$. Here, the accelerated MAGMA one-stage version is still faster.

For the tall 3:1 case in Figure 21(c), both LAPACK and MAGMA fare better, since part of the computation is in the initial QR factorization, which is primarily efficient Level 3 BLAS operations for all three implementations (LAPACK, MAGMA, and PLASMA). For the very tall 1000:1 matrices in Figures 21(e) and 21(f), PLASMA and MAGMA rely on their efficient QR factorization. In PLASMA, this is an implementation of the tall-skinny QR [1, 29], which even beats the accelerated MAGMA implementation.

Overall, we expected such an improvement using the two-stage technique, due to its heavy reliance on Level 3 BLAS. Even when performing more operations, it can still have an advantage.

I I.5. Energy Consumption. As we move toward exascale computing, power and energy consumption play increasingly critical roles. Figure 22 shows the power consumption over time during the SVD computation. We observe that PLASMA has the lowest energy consumption, due to its fast execution, despite having the highest power rate, indicative of its high compute intensity using Level 3 BLAS. Its energy consumption is about half that of LAPACK, and $23 \times$ less than EISPACK, as shown 


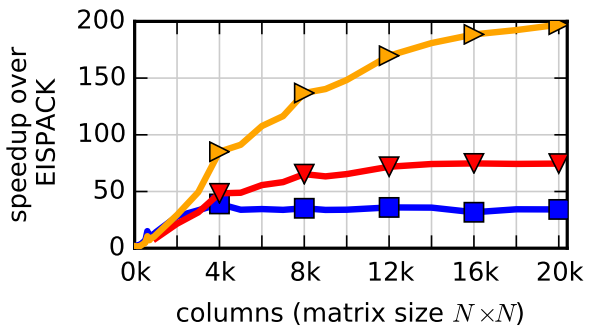

(a) square, no vectors

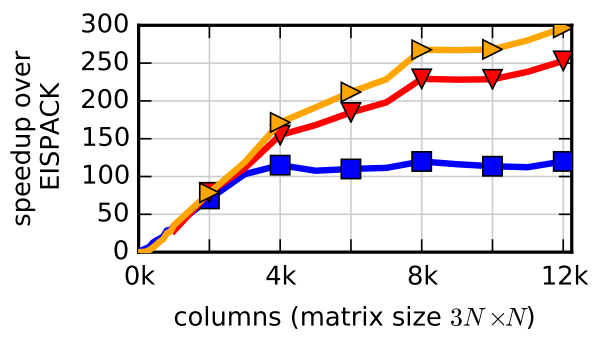

(c) tall, 3:1, no vectors

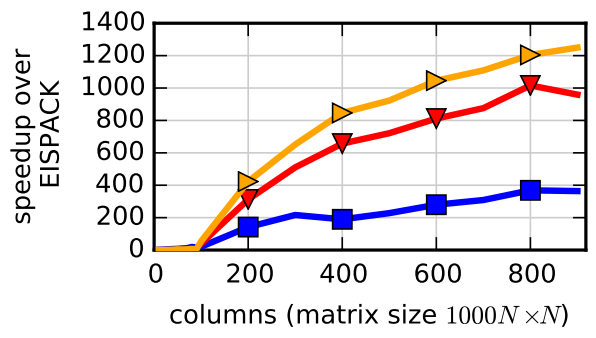

(e) tall, 1000:1, no vectors

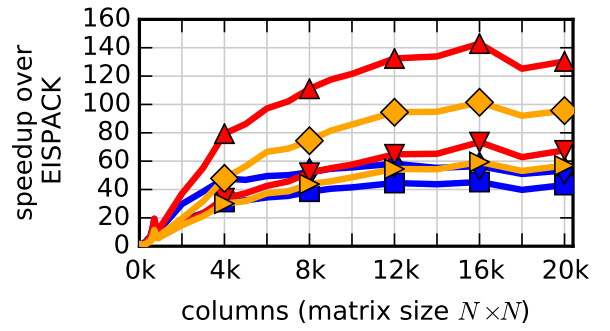

(b) square, with vectors

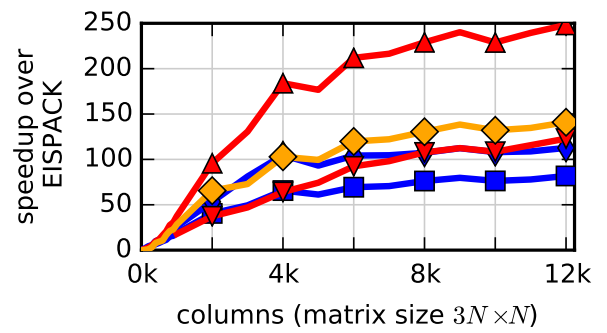

(d) tall, 3:1, with vectors

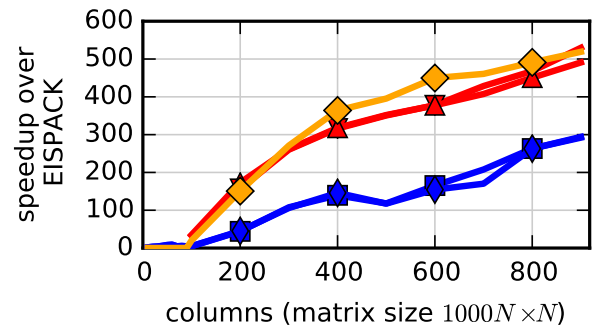

(f) tall, 1000:1, with vectors

\begin{tabular}{|llll|}
$\triangle$ & $\triangle$ MAGMA D\&C & $\triangleright$ & \\
$\nabla$ & $\nabla$ MAGMA QR iter. & PLASMA 2-stage, QR iter. \\
$\diamond$ & $\diamond$ PLASMA 2-stage, D\&C & & \\
\hline
\end{tabular}

Fig. 21 Comparison of MAGMA, PLASMA, and LAPACK.

in Figure 23(b). When computing singular values only, and no vectors, the difference is even more remarkable, with PLASMA being 5.6× more energy efficient than LAPACK, and 40× more energy efficient than EISPACK, as shown in Figure 23(a).

Interestingly, we can correlate the various phases of the computation with the power consumption. For LAPACK, the long plateau in Figure 22 up to the 105 seconds mark is the reduction to bidiagonal, followed by D\&C, where the power varies significantly, and ending with the two back transformations by $U_{1}$ and $V_{1}$ from the 130-150 seconds mark. In PLASMA, the reduction to bidiagonal is significantly shorter, up to the 20 seconds mark, followed by D\&C, and the back transformations by $U_{a}, U_{b}, V_{a}$, and $V_{b}$, which are twice as long as they are in LAPACK. EISPACK, in contrast, has a very long and steady computation. It uses only one core, 
SVD power for $\mathrm{N}=10000$ (with vectors)

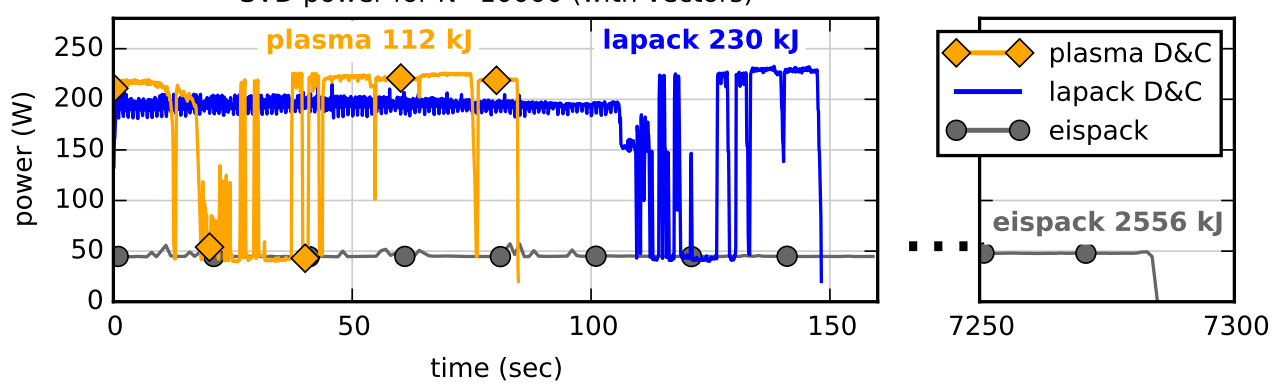

Fig. 22 Comparison of power during SVD computation for PLASMA, LAPACK, and EISPACK, for a square matrix of size $n=10000$. The total energy consumed during the computation is annotated for each one.

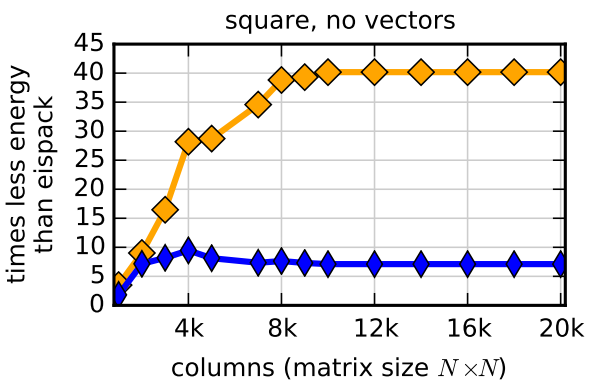

(a) square, no vectors

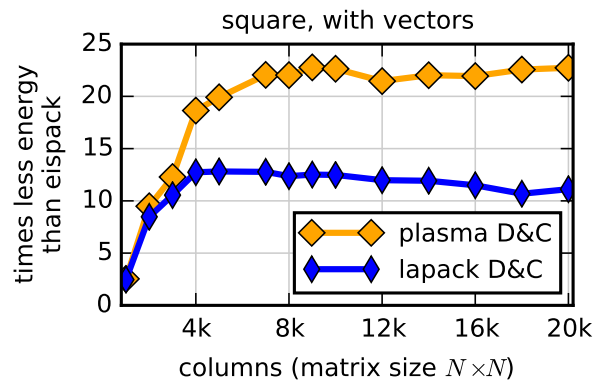

(b) square, with vectors

Fig. 23 Reduction in total energy consumption compared to EISPACK.

and thus has low power consumption; but the computation itself is $48 \times$ longer than LAPACK.

I I.6. MAGMA Accelerated Two-Stage Reduction. A two-stage algorithm can also be implemented very effectively using an accelerator. MAGMA accelerates the first-stage reduction to band form, as described above, and uses PLASMA for the second-stage reduction from band to bidiagonal. MAGMA also accelerates the computation of singular vectors, both applying the transformations from the second stage (e.g., $\left.U_{b} U_{2}\right)$ and applying the transformations from the first stage (e.g., $U_{a}\left(U_{b} U_{2}\right)$ ). Other steps are as in the accelerated one-stage MAGMA version. The profile in Figure 16 shows the difference with the one-stage version: the reduction to bidiagonal (blue with $\backslash \backslash$ hatching) is significantly reduced, but multiplying $U=U_{1} U_{2}=U_{a} U_{b} U_{2}$ and $V=V_{1} V_{2}=V_{a} V_{b} V_{2}$ (orange with // hatching) is increased.

Figure 24 shows the performance of the MAGMA two-stage implementation (dashed line), compared with the PLASMA two-stage and MAGMA one-stage implementations. The square, no vectors case in Figure 24(a) shows that for the bidiagonal reduction itself, the two-stage MAGMA is up to $2.4 \times$ faster than the two-stage PLASMA and $6.4 \times$ faster than the one-stage MAGMA, and nearly $500 \times$ faster than EISPACK. When computing singular vectors, in Figure 24(b), it is again up to $2.4 \times$ faster than PLASMA, but only $1.7 \times$ faster than the one-stage MAGMA, due to the 


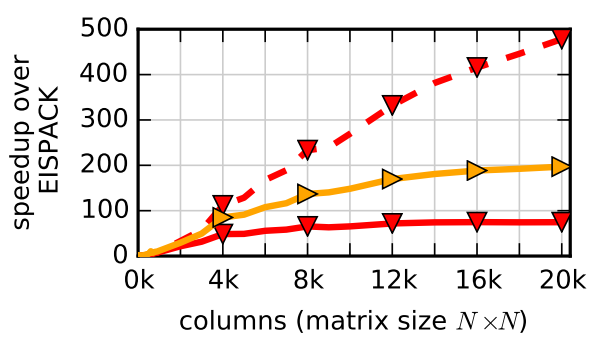

(a) square, no vectors

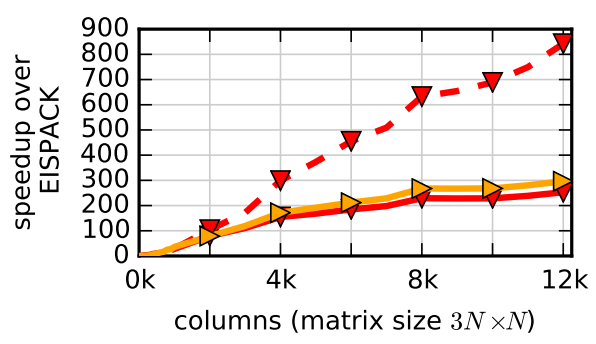

(c) tall, 3:1, no vectors

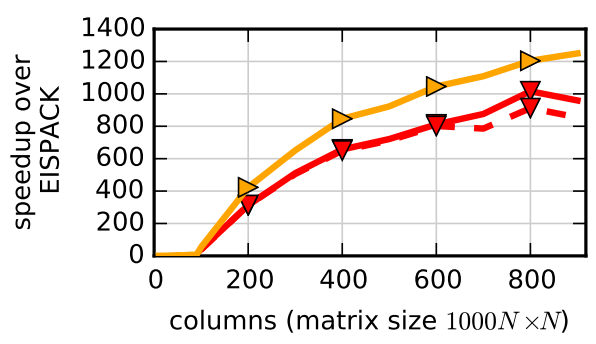

(e) tall, 1000:1, no vectors

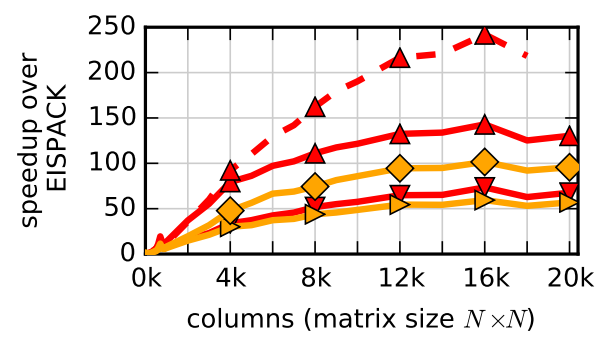

(b) square, with vectors

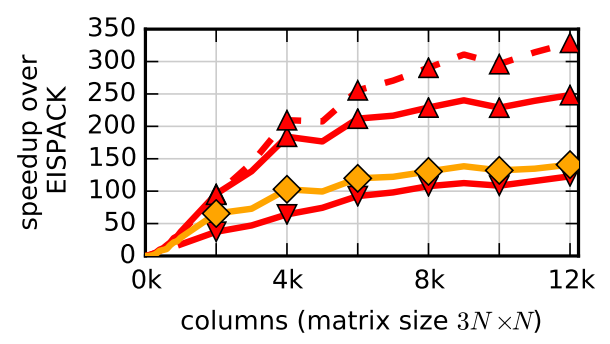

(d) tall, 3:1, with vectors

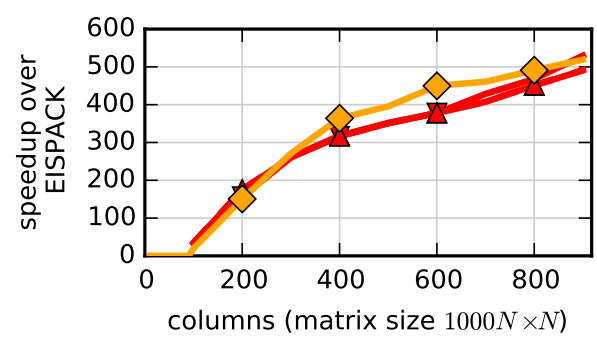

(f) tall, 1000:1, with vectors

Fig. 24 Comparison of MAGMA two-stage with MAGMA one-stage and PLASMA two-stage.

extra cost in multiplying by $U_{b}$ and $V_{b}$. It also performs well in the tall $3: 1$ case, while for the tall 1000:1 case, its time is dominated by the initial QR factorization, so it performs similarly to the one-stage MAGMA.

I I.7. DPLASMA Implementation for Distributed Memory. To cover the distributed-memory environment, we also performed a study on a modern, large distributed system. It is representative of a vast class of supercomputers commonly used for computationally intensive workloads. The DPLASMA algorithm is the two-stage algorithm described above for multicore, but implemented using the PaRSEC runtime engine $[19,18]$ to exploit the data flow representation, handle all the communication, and provide asynchronous task execution based on dependency analysis. PaRSEC 
employs the dataflow programming and execution model to provide a dynamic platform that can address the challenges posed by distributed hardware resources. The PaRSEC runtime combines the source program's task and dataflow information with supplementary information provided by the user - such as data distribution or hints about the importance of different tasks - and orchestrates task execution on the available hardware. From a technical perspective, PaRSEC is an event-driven system. When an event occurs, such as task completion, the runtime reacts by examining the dataflow to discover what future tasks can be executed based on the data generated by the completed task. The runtime handles the data exchange between distributed nodes, and thus it reacts to the events triggered by the completion of data transfers as well. Thus, communications become implicit and are handled automatically as efficiently as possible by the runtime. When no events are triggered because the hardware is busy executing application code, the runtime gets out of the way, allowing all hardware resources to be devoted to the application code's execution.

We benchmarked our two-stage implementation from the DPLASMA library and the ScaLAPACK SVD routine from Intel MKL. Because only the singular values computation of our two-stage approach is currently implemented in the distributed DPLASMA library, we limited our tests to the case where only the singular values are computed. We performed our experiment on a recent hardware system consisting of 49 distributed nodes, where every node has two sockets of 18-core Intel Xeon E5-2697 (Broadwell) processors, running at $2.6 \mathrm{GHz}$, providing a total of 1764 cores. Each socket has $35 \mathrm{MiB}$ of shared L3 cache, and each core has private $3.5 \mathrm{MiB}$ L2 and 448 $\mathrm{KiB}$ L1 caches. The system is equipped with $52 \mathrm{GiB}$ of memory per node. When only singular values are to be computed, the SVD solution consists of the reduction to bidiagonal and the computation of the singular values using QR iteration. Note that QR iteration on the bidiagonal matrix is a sequential process and thus it does not exploit any parallelism for either DPLASMA or ScaLAPACK. Its computational time is the same on either 1 or 49 nodes, and this time increases quadratically with the matrix size. Thus, the percentage of time spent in this portion varies with the matrix size. QR iteration consists of less than $5 \%$ of the time for a matrix of size $20 \mathrm{k}$, while it reaches about $15 \%$ for ScaLAPACK and $26 \%$ for DPLASMA for a matrix of size $200 \mathrm{k}$. As a result, the speedup will be affected by this constant,

$$
\text { speedup }=\frac{\text { time }_{\text {DPLASMA-BRD }}+t_{x}}{\text { time } \text { SCALAPACK-BRD }+t_{x}},
$$

where $t_{x}$ is the time required to perform the bidiagonal singular value computation. Figure 25 shows the comparison between our implementation and the ScaLAPACK pdgesvd. Asymptotically, our code achieves up to a $3 \times$ speedup for the largest matrices tested. This is the result of the efficient implementation of the first stage (reduction to band) using the PaRSEC engine, which enables us to exploit the compute-intensive nature of this stage, thereby minimizing the communication cost, and also from the careful design and implementation of the second stage that maps both the algorithm and the data to the hardware using cache-friendly kernels and data-locality-aware scheduling. Note that for small matrix sizes (e.g., a matrix of size 20k), there is not enough parallelism to exploit the 1764 available cores to make our two-stage algorithm $3 \times$ faster; the tile size is about 160 , so there are only about 125 tiles in each direction.

I2. Jacobi Methods. In contrast to bidiagonalization methods, Jacobi methods operate on the entire matrix $A$, without ever reducing to bidiagonal. This allows Jacobi methods to attain high relative accuracy, which will be discussed in section 13 . 


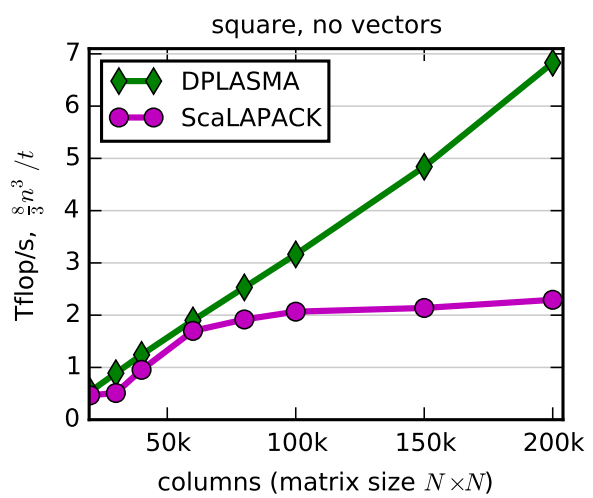

(a) square, no vectors, performance

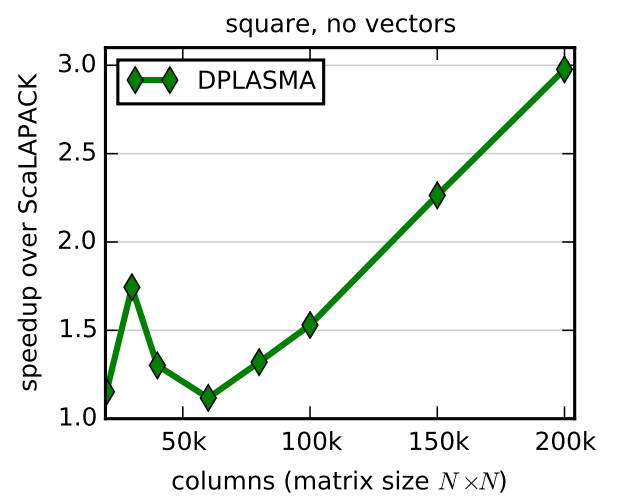

(b) square, no vectors, speedup

Fig. 25 Comparison of DPLASMA and ScaLAPACK computing singular values only for square matrices on 49 nodes (1764 cores).

Jacobi first proposed his method in 1848 for solving the symmetric eigenvalue problem [73] by diagonalizing the matrix $A$ using a sequence of plane rotations

$$
A_{(0)}=A, \quad A_{(k+1)}=J_{(k)}^{T} A_{(k)} J_{(k)}, \quad A_{(k)} \rightarrow \Lambda \text { as } k \rightarrow \infty .
$$

Each plane rotation, $J_{(k)}=J_{(k)}(i, j, \theta)$, now called a Jacobi or Givens rotation, is an orthogonal matrix that differs from the identity only in rows and columns $i$ and $j$,

$$
J(i, j, \theta)=\left[\begin{array}{ccccc}
I & & & & \\
& c & & s & \\
& & I & & \\
& -s & & c & \\
& & & & I
\end{array}\right],
$$

where $c=\cos \theta$ and $s=\sin \theta$. The angle $\theta$ is chosen to eliminate the pair $a_{i j}, a_{j i}$ by applying $J(i, j, \theta)$ on the left and right of $A$, which can be viewed as the $2 \times 2$ eigenvalue problem

$$
\hat{J}_{(k)}^{T} \hat{A}_{(k)} \hat{J}_{(k)}=\left[\begin{array}{cc}
c & s \\
-s & c
\end{array}\right]^{T}\left[\begin{array}{cc}
a_{i i} & a_{i j} \\
a_{j i} & a_{j j}
\end{array}\right]\left[\begin{array}{cc}
c & s \\
-s & c
\end{array}\right]=\left[\begin{array}{cc}
d_{i i} & 0 \\
0 & d_{j j}
\end{array}\right]=\hat{A}_{(k+1)},
$$

where the notation $\hat{A}$ is the $2 \times 2$ submatrix $\left[\begin{array}{cc}a_{i i} & a_{i j} \\ a_{j i} & a_{j j}\end{array}\right]$ of matrix $A$. Subsequent eliminations will fill in the eliminated entry, but at each step the norm of off-diagonal elements,

$$
\operatorname{off}(A)=\|A-\operatorname{diag}(A)\|_{F}=\left(\sum_{i \neq j} a_{i j}^{2}\right)^{1 / 2}
$$

is reduced until the matrix converges to diagonal form, $\Lambda$, revealing the eigenvalues. Accumulating the plane rotations, $V=J_{(0)} J_{(1)} \ldots$, yields the eigenvectors. Originally, Jacobi chose to eliminate the off-diagonal pair $a_{i j}, a_{j i}$ of largest magnitude at each step, giving the largest possible reduction in off $(A)$. This is inefficient as it introduces an $O\left(n^{2}\right)$ search for each rotation of $O(n)$ work. Instead, in modern times 


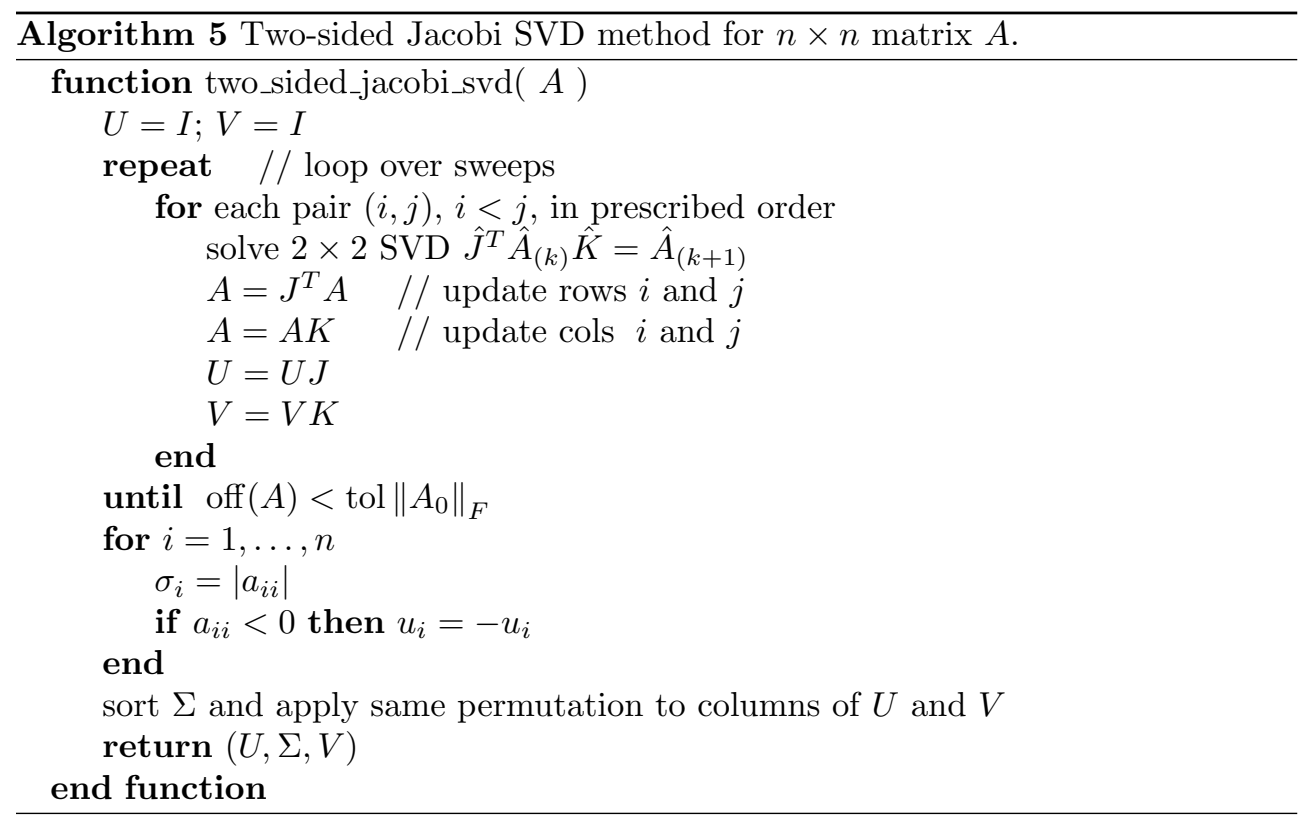

the method was reformulated so that one sweep goes over all $n(n-1) / 2$ combinations of $(i, j)$ with $i<j$ in a predetermined order, typically cyclic by rows, i.e.,

$$
(1,2),(1,3), \ldots,(1, n) ;(2,3), \ldots,(2, n) ; \ldots ;(n-1, n),
$$

or cyclic by columns. It converges after a small number of sweeps, typically $5-10$. Wilkinson [116] showed that convergence is ultimately quadratic. Rutishauser [102] gave a robust implementation in the Wilkinson-Reinsch Handbook [117].

12.I. Two-Sided Jacobi SVD. Jacobi's eigenvalue method was generalized to the SVD of a general, nonsymmetric matrix in two different ways. The first way is the twosided method due to Kogbetliantz [76], which applies two different plane rotations, $J(i, j, \theta)$ on the left of $A$ and $K(i, j, \phi)$ on the right of $A$, to eliminate the $a_{i j}$ and $a_{j i}$ entries. As before, sweeps are done over the off-diagonal entries until the norm of off-diagonal entries is below a specified tolerance, revealing the singular values, $\Sigma$, via the iteration

$$
A_{(0)}=A, \quad A_{(k+1)}=J_{(k)}^{T} A_{(k)} K_{(k)}, \quad A_{(k)} \rightarrow \Sigma \text { as } k \rightarrow \infty .
$$

Accumulating the left rotations, $U=J_{(0)} J_{(1)} \ldots$, gives the left singular vectors, while accumulating the right rotations, $V=K_{(0)} K_{(1)} \ldots$, gives the right singular vectors.

Determining $J(i, j, \theta)$ and $K(i, j, \phi)$ can be viewed as solving a $2 \times 2$ SVD problem,

$$
\hat{J}_{(k)}^{T} \hat{A}_{(k)} \hat{K}_{(k)}=\left[\begin{array}{cc}
c_{J} & s_{J} \\
-s_{J} & c_{J}
\end{array}\right]^{T}\left[\begin{array}{ll}
a_{i i} & a_{i j} \\
a_{j i} & a_{j j}
\end{array}\right]\left[\begin{array}{cc}
c_{K} & s_{K} \\
-s_{K} & c_{K}
\end{array}\right]=\left[\begin{array}{ll}
d_{i i} & \\
& d_{j j}
\end{array}\right]=\hat{A}_{(k+1)} .
$$

The angles for $J$ and $K$ are not uniquely determined, so various methods have been derived $[22,49,76]$. Brent, Luk, and Van Loan [22] proposed the algorithm USVD, which uses one rotation to symmetrize the $2 \times 2$ subproblem, then a second rotation to eliminate the off-diagonal entries. This produces an unnormalized SVD, where the 
diagonal entries are unsorted and may be negative. Postprocessing to sort and adjust the signs of the singular values and singular vectors yields a standard SVD. They also formulated the normalized rotation/reflection algorithm NSVD that corrects the signs during the iteration. Algorithm 5 outlines the two-sided Jacobi method.

Rectangular matrices can be handled by first doing a QR factorization, optionally with pivoting, and then doing the SVD of the $R$ matrix, as previously described for bidiagonalization methods (subsection 5.4). For Jacobi, this QR factorization has the added benefit of preconditioning the system to converge faster, as discussed further in subsection 12.5 .

Heath et al. [67] developed a variant for computing the SVD of a product of matrices, $A=B^{T} C$, without explicitly forming $A$. Applying rotations $B_{(k+1)}=B_{(k)} J$ and $C_{(k+1)}=C_{(k)} K$ implicitly applies $J$ and $K$ on both sides of $A$. When $B=C$, it simplifies to the one-sided Jacobi method, discussed next.

12.2. One-Sided Jacobi. The second way to generalize the Jacobi method to the SVD is a one-sided method due to Hestenes [68]. Earlier we noted that the SVD can be solved by computing the eigenvalues of the Gram matrix, $A^{T} A$, but that explicitly forming $A^{T} A$ is undesirable for numerical reasons. Instead, Hestenes applied plane rotations on only the right side of $A$ to orthogonalize the columns of $A$, which implicitly performs the two-sided Jacobi eigenvalue method on $A^{T} A$. The columns of $A$ converge to $U \Sigma$, that is, the left singular vectors scaled by the singular values:

$$
A_{(0)}=A, \quad A_{(k+1)}=A_{(k)} J_{(k)}, \quad A_{(k)} \rightarrow U \Sigma \text { as } k \rightarrow \infty .
$$

This means that, implicitly, $A_{(k)}^{T} A_{(k)} \rightarrow \Sigma^{2}$. Accumulating the rotations, $V=$ $J_{(0)} J_{(1)} \ldots$, gives the right singular vectors. Alternatively, $V$ can be solved for after the iteration, as described below in subsection 12.5.

The rotations are determined similarly to the Jacobi eigenvalue method, by solving the $2 \times 2$ eigenvalue problem

$$
\hat{J}_{(k)}^{T}\left[\begin{array}{ll}
b_{i i} & b_{i j} \\
b_{i j} & b_{j j}
\end{array}\right] \hat{J}_{(k)}=\left[\begin{array}{ll}
d_{i i} & \\
& d_{j j}
\end{array}\right],
$$

where $b_{i j}=a_{i}^{T} a_{j}$ and $a_{i}$ is the $i$ th column of $A_{(k)}$. Over the course of a sweep, it computes the matrix $B=A^{T} A$; however, $J$ is not applied directly to $A^{T} A$, but to $A$ itself, avoiding the numerical instabilities associated with $A^{T} A$. Algorithm 6 outlines the one-sided Jacobi method. It skips rotations if $\left|b_{i j}\right|<\epsilon \sqrt{b_{i i} b_{j j}}$, indicating that columns $a_{i}$ and $a_{j}$ are already numerically orthogonal. It converges when all rotations in a sweep are skipped. Using this formula to check for convergence is required for attaining high relative accuracy [32] (see section 13). The $b_{i i}$ column norms can be cached rather than recomputed for each pair, which reduces operations when rotations are skipped. Note that the last sweep takes about $n^{3}$ flops computing $b_{i j}$ terms to check for convergence, without doing any useful work.

A left-handed version can be defined analogously by applying rotations on the left to orthogonalize the rows of $A$ [84]. This might be preferred if $A$ is a wide matrix stored row-wise, rather than a tall matrix stored column-wise.

One-sided Jacobi can be applied to a rectangular matrix, but again, preprocessing using a QR factorization, and applying Jacobi on the square $R$ matrix, reduces the operation count and preconditions the system for faster convergence; see subsection 12.5 . 


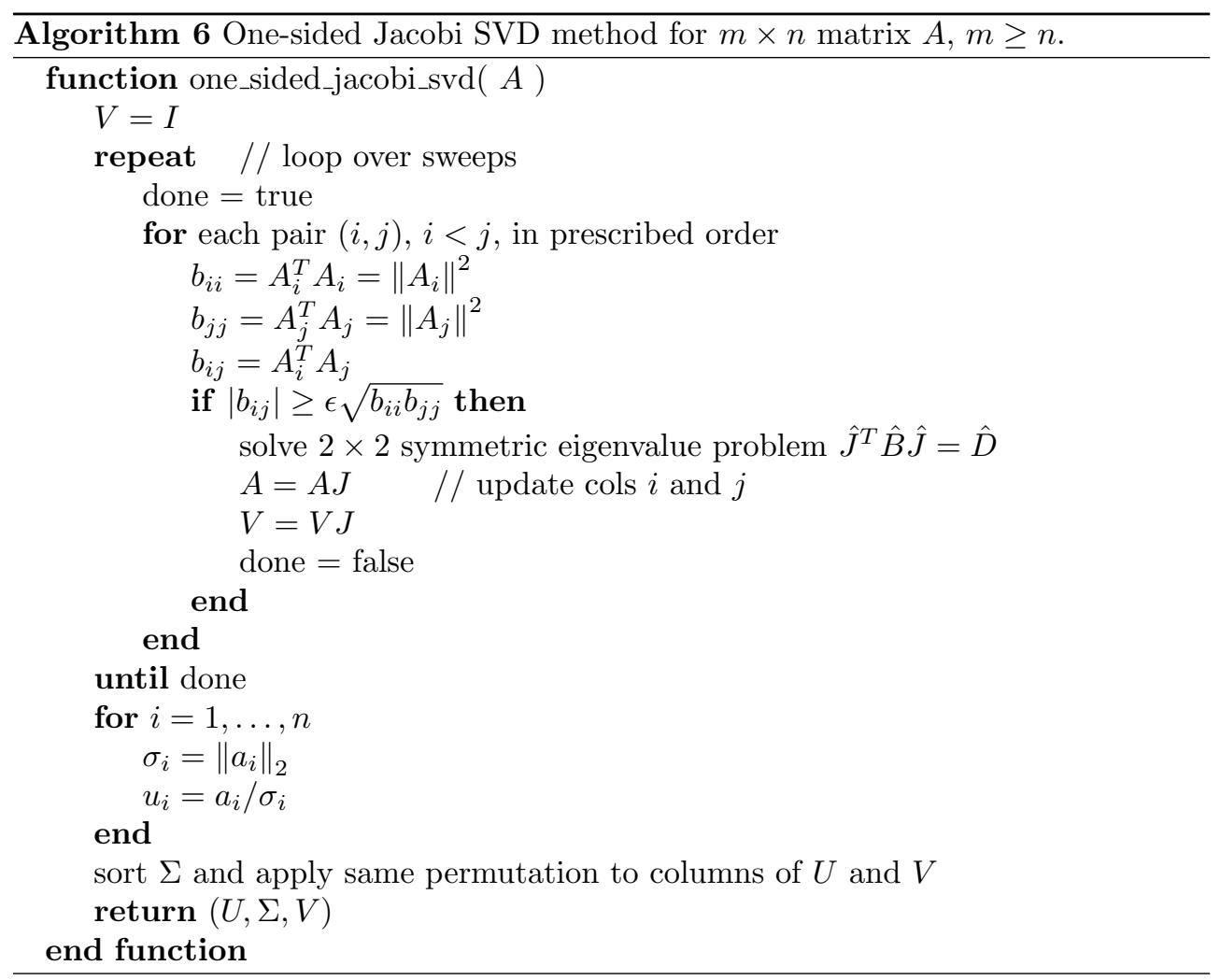

12.3. Convergence. For the row and column cyclic orderings, Forsythe and Henrici [49] proved that all the Jacobi methods (two-sided eigenvalue, one-sided SVD, and two-sided SVD) converge, provided the rotation angles are bounded below $\pi / 2$ by some $b$,

$$
|\theta| \leq b<\pi / 2 .
$$

For the two-sided eigenvalue and one-sided SVD methods, $\theta$ can always be chosen to satisfy (10); see [102]. For the two-sided SVD method, however, this condition may fail to hold. In Forsythe and Henrici's method, the bound is $b=\frac{\pi}{2}$, which may introduce a cycle interchanging two singular values without converging. For the methods of Brent, Luk, and Van Loan [22], NSVD has a bound $b=3 \pi / 4$ and USVD has a bound $b=5 \pi / 4$. Proofs for other orderings, particularly for parallel orderings, have been elusive. Despite failing to satisfy the convergence proof's prerequisites, in practice Jacobi methods reliably converge. Using a threshold to skip updates to small entries is a common tactic, especially in the first several sweeps, to accelerate and guarantee convergence $[102,27,8]$.

When applied to triangular matrices, Heath et al. [67] and Hari and Veselić [66] observed that applying one sweep of the two-sided SVD method with the row-cyclic ordering (without thresholding) converts an upper-triangular matrix to lower triangular, and vice versa. Hari and Veselić derived rotation angle formulas in the triangular case, and proved that the angles are bounded below $\pi / 2$, guaranteeing convergence. Hari and Matejaš [65] later derived more accurate formulas. 


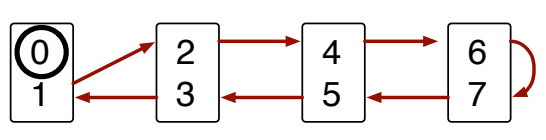

(a) round-robin

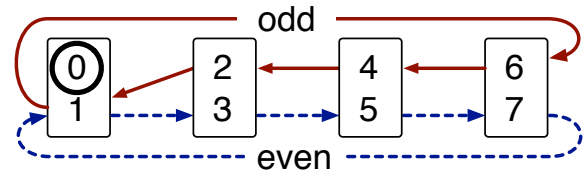

(b) odd-even

Fig. 26 Parallel orderings. Rectangles indicating processors are labeled with their assigned columns. Arrows depict movement of columns between Jacobi sweeps. The circled pivot column is stationary.

Applying column pivoting during the Jacobi iterations can improve convergence. In the one-sided method, de Rijk [27] follows the row-cyclic ordering, but at the start of row $i$, searches columns $i, \ldots, n$ for the column of largest norm and pivots it to column $i$. Unfortunately, using the row-cyclic ordering makes parallelism difficult. Zhou and Brent [120] likewise show that sorting column norms improves convergence, and they give a parallel ordering for sorting.

1 2.4. Parallel Orderings. In two-sided Jacobi, a pair of rotations applied on the left and right affect only two rows and two columns. In one-sided Jacobi, each rotation applied on the right affects only two columns. Therefore, in both cases, $\lfloor n / 2\rfloor$ rotations can be performed in parallel. However, the row and column cyclic orderings are not amenable to parallel computation, as they introduce dependencies between consecutive pairs of elements. Since there are $n(n-1) / 2$ pairs to eliminate, an optimal parallel ordering would have $n-1$ steps, with each step eliminating $n / 2$ pairs in parallel (for $n$ even). Many different parallel Jacobi orderings have been devised. While parallel orderings typically lack a proof of convergence, in practice they work reliably.

Commonly, for parallel implementations of both one-sided and two-sided Jacobi, the matrix is distributed by columns. Early systolic implementations placed two columns [21] or a $2 \times 2$ submatrix [22] per processor. Later block implementations placed two block columns [15, 11] or a $2 \times 2$ block submatrix [12] per processor. When each processor stores two columns, one-sided Jacobi has the advantage that no communication is required during an update, whereas in two-sided Jacobi, the left transformations ( $J$ 's) must be broadcast in an all-to-all fashion.

Brent and Luk [21] introduced the round-robin ordering, shown in Figure 26(a), which had previously been known for chess tournaments. After each Jacobi rotation, each node sends and receives two columns, except for the pivot node that sends and receives one column. Eberlein [46] gave the odd-even ordering in Figure 26(b). After each odd sweep, the odd permutation (solid arrows) is used; after even sweeps, the even permutation (dashed arrows) is used. Each node sends and receives one column.

Luk and Park [85] studied the equivalence of orderings, demonstrating that many orderings are equivalent in the sense that relabeling the columns gives identical orderings. For example, choosing a different pivot column in round-robin will give an equivalent ordering. Luk and Park showed that the two main classes of Jacobi orderings are the round-robin and odd-even types. Bečka and Vajteršic $[12,11]$ compared implementations of the round-robin, odd-even, and a butterfly-like ordering inspired by the fast Fourier transform (FFT), on ring, hypercube, and mesh networks for block Jacobi methods. 


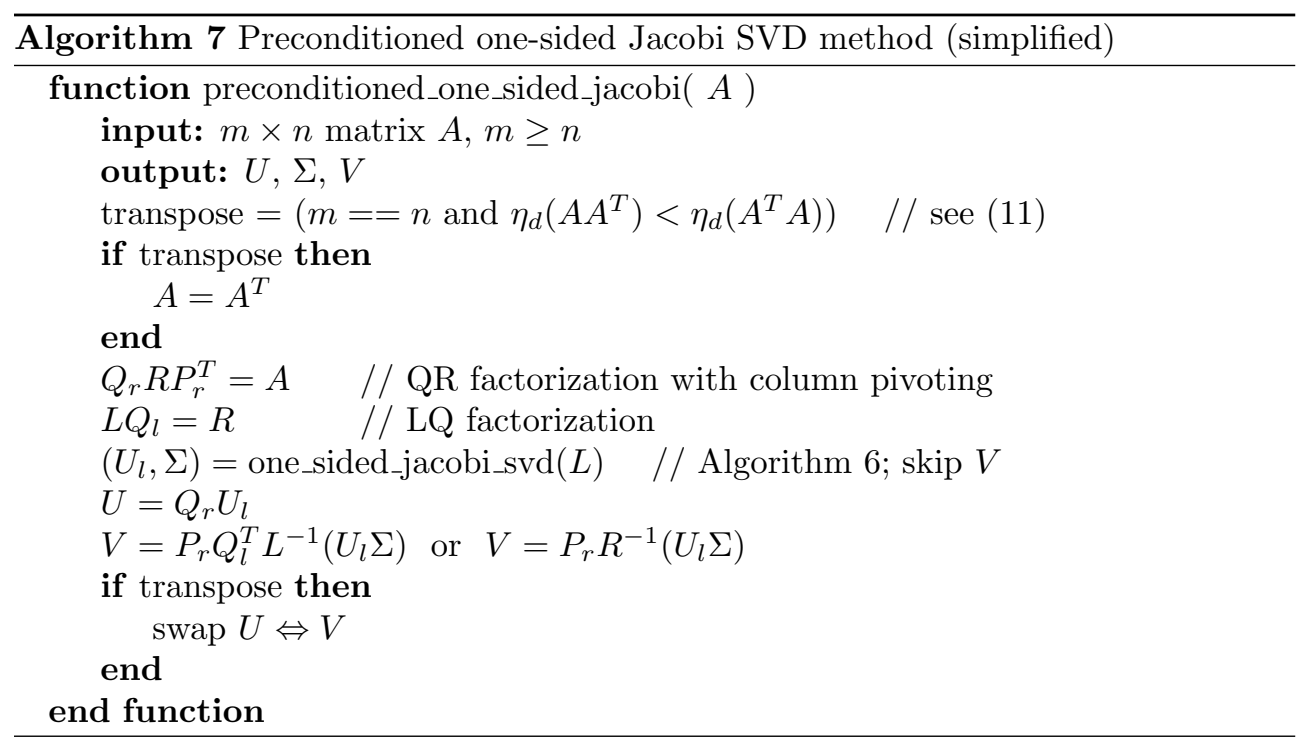

12.5. Preconditioning. Another means to improving the speed of Jacobi methods is to precondition the matrix to reduce the number of sweeps required for convergence. Drmač and Veselić [44] introduced several forms of preconditioning for the one-sided Jacobi method. The major ideas are outlined below, with a simplified version in Algorithm 7.

First, for a square matrix $A$, heuristically choose to factor either $X=A$ or $X=A^{T}$. Drmač and Veselić give the example of $A=D Q$, where $D$ is diagonal and $Q$ is orthogonal. One-sided Jacobi applied to $A$ implicitly diagonalizes $Q^{T} D^{2} D$, while applied to $A^{T}$, it implicitly diagonalizes $D^{2}$, which is already diagonal. One heuristic they suggest is to choose the $X$ that maximizes $\left\|\operatorname{diag}\left(X^{T} X\right)\right\|_{2}$, hence minimizing off $\left(X^{T} X\right)$. Their second heuristic is to choose the $X$ that minimizes the diagonal entropy of $X^{T} X$, defined by

$$
\eta_{d}\left(X^{T} X\right)=\eta\left(\operatorname{diag}\left(X^{T} X\right) / \operatorname{trace}\left(X^{T} X\right)\right),
$$

where the entropy of a vector $p$ with $p_{i} \geq 0, \sum_{i} p_{i}=1$, is defined as

$$
\eta(p)=-\frac{1}{\log n} \sum_{i=1}^{n} p_{i} \log p_{i} \quad \text { with } 0 \log 0 \equiv 0
$$

Both heuristics are $O\left(n^{2}\right)$.

The second preconditioning technique is to use a $\mathrm{QR}$ factorization with column pivoting (QRP) of $A$, then factor $R$. This concentrates the mass of the matrix along the diagonal of $R^{T} R$, reducing the number of Jacobi sweeps. For a rectangular $m \times n$ problem, $m>n$, this also shrinks it to an $n \times n$ problem, as in subsection 5.4.

Third, use either an LQ factorization of $R$, or simply let $L=R^{T}$, then factor $L$. An LQ factorization further concentrates the mass along the diagonal of $L^{T} L$. Using LQ is particularly advantageous in the rank deficient case. For a matrix of rank $r$, QRP generates $R=\left[\begin{array}{cc}R_{11} & R_{12} \\ 0 & R_{22}\end{array}\right]$ with the $(n-r) \times(n-r)$ block $R_{22}$ being negligible. Doing an LQ factorization of $\left[\begin{array}{ll}R_{11} & R_{12}\end{array}\right]$ yields a smaller, $r \times r$, full-rank 
matrix $L$. Alternatively, simply using $L=R^{T}$ is an implicit step of Rutishauser's LR diagonalization applied to $R^{T} R$, again concentrating mass along the diagonal of $L^{T} L$ as compared to $R^{T} R$.

Additionally, Drmač and Veselić's error analysis based on using QRP and optionally LQ factorization shows that computing $V$ by solving with either of the triangular matrices $L$ or $R$ is numerically stable and generates an orthogonal matrix; see Algorithm 7 for specifics. This allows us to skip accumulating $V$ during the one-sided Jacobi iteration, removing some Level 1 BLAS operations, and adding Level 3 BLAS operations after the iteration, so we can expect a good performance increase. Their paper gives detailed algorithms that make choices about which preconditioning to use based on condition estimates. Hari [64] and Bečka, Okša, and Vajteršic [9] also applied QRP and LQ preconditioning in the context of parallel one-sided block Jacobi.

In addition to preconditioning, Drmač and Veselić [45] introduced optimizations in the one-sided Jacobi iteration, based on the structure of the preconditioned matrix. In the first sweep, the zero structure of the triangular matrix can be exploited to reduce computation. Second, based on work by Mascarenhas [87], they used a modified rowcyclic strategy to more frequently visit diagonal blocks, since those blocks converge at a slower rate. Heuristically, based on the expectation that $L^{T} L$ is diagonally dominant, during the first few sweeps, if two rotations in a row are skipped due to thresholding, they skip the rest of the row. This avoids computing dot products when the rotation will likely be skipped. Finally, they used a tiled row-cyclic strategy to improve cache efficiency. All of these improvements combine for a more efficient algorithm.

Okša and Vajteršic [95] showed that the same preconditioning techniques, QRP factorization optionally followed by LQ factorization, also improve convergence for the parallel two-sided block Jacobi method. In their tests, preconditioning concentrated more than $99 \%$ of the weight of $\|A\|_{F}$ into the diagonal blocks. Depending on the $\mathrm{SVD}$, this gave up to an order-of-magnitude reduction in time. This preconditioning was later extended to multiple QR iterations [10].

As noted earlier, two-sided Jacobi preserves the triangular structure when used with an appropriate cyclic ordering. Hari and Matejaš $[65,88,89]$ use the QRP and LQ preprocessing to generate triangular matrices. They prove high relative accuracy results for the two-sided Jacobi method on such triangular matrices, and utilize a parallel ordering due to Sameh [103] that preserves the triangular structure.

12.6. Block Jacobi. In section 5 , we saw that blocking was a major improvement for SVD methods. Blocking can also be favorably applied to Jacobi methods. Van Loan [112] and Bischof [15] were among the first to describe two-sided block Jacobi SVD methods. The method is very similar to the nonblock implementation, with plane rotations $J$ and $K$ operating on two rows or columns now becoming orthogonal block rotations operating on two block rows or block columns. For a block size $n_{b}$, let $N=\left\lceil n / n_{b}\right\rceil$ be the number of blocks. The indices $i, j$ now loop over the blocks, $1, \ldots, N$. We reinterpret the notation $\hat{A}$ to be the $2 \times 2$ block matrix

$$
\hat{A}=\left[\begin{array}{cc}
A_{i i} & A_{i j} \\
A_{j i} & A_{j j}
\end{array}\right]
$$

where each $A_{i j}$ is an $n_{b} \times n_{b}$ block. Instead of the $2 \times 2 \operatorname{SVD}(8)$, it computes a $2 \times 2$ block SVD,

$$
\hat{J}^{T} \hat{A} \hat{K}=\hat{J}^{T}\left[\begin{array}{cc}
A_{i i} & A_{i j} \\
A_{j i} & A_{j j}
\end{array}\right] \hat{K}=\left[\begin{array}{cc}
D_{i i} & 0 \\
0 & D_{j j}
\end{array}\right]
$$


either recursively using a serial Jacobi method, or using some other SVD method such as QR iteration. Each processor now holds two block columns. Block row and column updates by the orthogonal matrices $J$ and $K$ are applied as Level 3 BLAS matrix multiplies, greatly enhancing the efficiency of the algorithm.

Bischof [15] investigated two methods to solve the SVD subproblem: using QR iteration or using a single sweep of two-sided Jacobi. In the latter case, in using only one sweep the block method does not fully annihilate the off-diagonal blocks of the $2 \times 2$ block subproblem, and is in fact simply a reorganization of the nonblock method, but with updates applied using Level 3 BLAS. Bischof found that using Jacobi to solve the subproblem was faster than using QR iteration; however, this was prior to the fast blocked versions of QR iteration available in LAPACK.

Arbenz and Slapničar [5] gave an early implementation for the one-sided block Jacobi SVD method. Again, the block method is very similar to the nonblock method, with the $2 \times 2$ eigenvalue problem (9) being replaced with a $2 \times 2$ block eigenvalue problem,

$$
\hat{J}^{T} \hat{A} \hat{J}=\hat{J}^{T}\left[\begin{array}{cc}
B_{i i} & B_{i j} \\
B_{i j}^{T} & B_{j j}
\end{array}\right] \hat{J}=\left[\begin{array}{cc}
D_{i i} & 0 \\
0 & D_{j j}
\end{array}\right]
$$

with $B_{i j}=A_{i}^{T} A_{j}$, where $A_{i}$ is the $i$ th block column of $A$. Arbenz and Slapničar used the two-sided Jacobi eigenvalue method to solve the subproblem, which is important for preserving Jacobi's high relative accuracy. Hari [64] derived an optimization using the cosine-sine decomposition as a kind of "fast scaled block-rotation," reducing the flop count up to $40 \%$. Boukaram et al. [20] developed batched one-sided Jacobi and block Jacobi methods for GPUs, to compute SVD factorizations of a batch of small matrices.

Bečka, Okša, and Vajteršic introduced dynamic orderings for the two-sided [8] and one-sided [9] Jacobi methods. Instead of using a cyclic ordering such as row-cyclic, round-robin, or odd-even, the idea is to find the off-diagonal blocks of maximum norm to eliminate. This is Jacobi's original idea, applied on the block level. Using a greedy solution to the maximum-weight perfect matching problem takes $O\left(p^{2} \log p\right)$ time for $p$ processors and yields a set of $N / 2$ subproblems of maximum weight to solve in parallel. Their results show significantly improved convergence and time to solution.

12.7. Performance Analysis. While Jacobi methods have a long history, even predating bidiagonalization methods, many implementations have been either research codes or designed for unique systems like the ILLIAC IV [84]. Therefore, we do not have as rich a collection of historical implementations to compare as for bidiagonalization methods. We tested four current implementations of Jacobi methods:

- One-sided Jacobi, available in LAPACK as dgesvj, due to Drmač [44].

- Preconditioned one-sided Jacobi, available in LAPACK as dgejsv, due to Drmač [44].

- Two-sided Jacobi, available in Eigen 3.3.3 [47].

- Preconditioned one-sided block Jacobi, due to Bečka, Okša, and Vajteršic [9].

Jacobi has traditionally trailed bidiagonalization methods in performance for two reasons. First, a comparison of flops in Table 2 shows that for computing singular values only (no vectors), Jacobi cannot finish even one sweep in the same flops as bidiagonalization $\left(\frac{8}{3} n^{3}\right)$. When computing vectors, Jacobi would need to complete in two sweeps to have fewer flops than QR iteration, and one sweep to have fewer flops than D\&C. However, with optimizations to skip rotations and take advantage of matrix structure $[45,89]$, these Jacobi flop counts are significant overestimates.

Copyright $@$ ㅇ by SIAM. Unauthorized reproduction of this article is prohibited. 
Table 2 Floating-point operation counts for square $n \times n$ matrix and $S$ Jacobi sweeps. For Jacobi, fast Givens rotations [63] are assumed. For preconditioned Jacobi, initial QRP and LQ factorizations and triangular solve for $V$ are also assumed.

\begin{tabular}{lll}
\hline & No vectors & With vectors \\
\hline QR iteration & $\frac{8}{3} n^{3}$ & $\frac{52}{3} n^{3} \approx 17 n^{3}$ \\
D\&C & $\frac{8}{3} n^{3}$ & $\frac{28}{3} n^{3} \approx 9 n^{3}$ \\
One-sided Jacobi & $5 S n^{3}$ & $7 S n^{3}$ \\
Two-sided Jacobi & $4 S n^{3}$ & $8 S n^{3}$ \\
Preconditioned one-sided Jacobi & $5 S n^{3}+\frac{8}{3} n^{3}$ & $5 S n^{3}+\frac{17}{3} n^{3}$ \\
Preconditioned two-sided Jacobi & $4 S n^{3}+\frac{8}{3} n^{3}$ & $6 S n^{3}+\frac{17}{3} n^{3}$ \\
\hline
\end{tabular}

However, as we have repeatedly seen, flops are now a poor metric for performance. It matters whether flops are in compute-intensive Level 3 BLAS or not. For Jacobi, dot products and plane rotations are Level 1 BLAS, so are memory bandwidth limited. For preconditioned Jacobi, QRP has a mixture of Level 2 and Level 3 BLAS operations, similar to the traditional one-stage bidiagonalization discussed in subsection 5.1, so its performance is also limited by memory bandwidth. The triangular solve for $V$ and multiplying $Q U$ will both be Level 3 BLAS operations. The level of parallelism also matters. The two LAPACK implementations, one-sided Jacobi and preconditioned one-sided Jacobi, do not use explicit parallelism. Therefore, the only parallelism is within the BLAS, which is very limited for Level 1 BLAS. In contrast, the block Jacobi method uses Level 3 BLAS operations and explicit parallelism via MPI, so we can expect much better performance.

In Figure 27(a), for square matrices without vectors, both one-sided Jacobi methods were about half EISPACK's speed, while with vectors in Figure 27(b), preconditioned Jacobi is $2 \times$ faster than plain Jacobi, and close to EISPACK's speed. For tall, 3:1 matrices in Figure 27(c), the plain one-sided Jacobi does not do an initial QR factorization, so it remains about half of EISPACK's speed, while the preconditioned Jacobi improves to about $2 \times$ EISPACK's speed. When computing vectors in Figure 27(d), the preconditioned Jacobi version gains even more, being over $3 \times$ faster than EISPACK.

For the very tall-skinny 1000:1 case in Figures 27(e) and 27(f), the time with preconditioned Jacobi is dominated by QRP, which uses more Level 2 and 3 BLAS operations, so the performance improves to over $100 \times$ EISPACK. LAPACK's QR iteration uses a regular QR factorization (no pivoting), which is predominantly Level 3 BLAS, so its performance is significantly faster than Jacobi. However, QRP will generate a more accurate factorization than regular QR, especially if $A$ is ill-conditioned.

In most cases, the Jacobi single-core performance was identical to its multicore performance, indicating that the Level 1 BLAS routines do not have appreciable parallelism. For tall matrices, preconditioning gained an advantage when using multiple cores, shown by the difference between the $\longrightarrow$ and $\longrightarrow$ lines in Figures 27(c) to $27(\mathrm{f})$, due to parallelism within QRP, solving for $V$, and computing $Q U$.

In all of these results, the two-sided Jacobi implementation available in Eigen was considerably slower. This can partly be explained because it has to update the matrix 


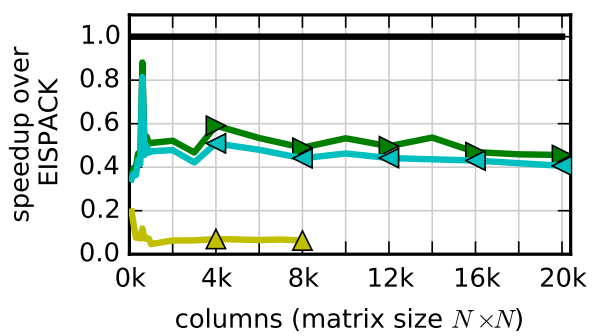

(a) square, no vectors

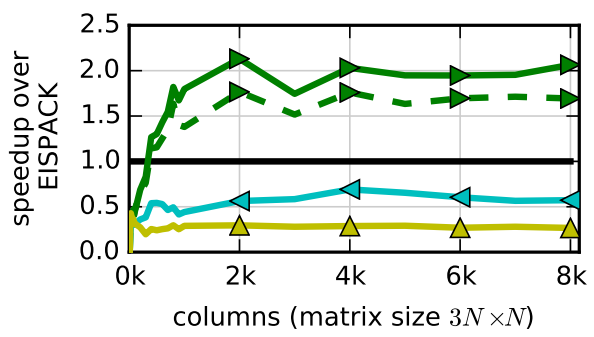

(c) tall, 3:1, no vectors

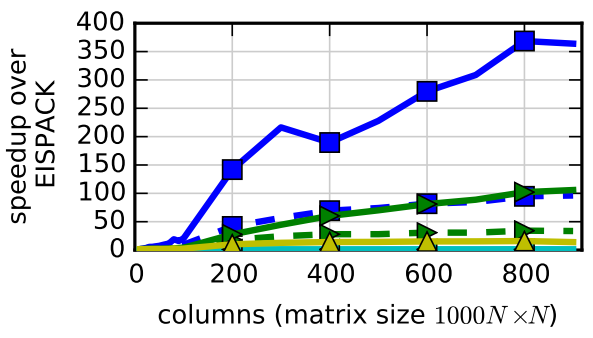

(e) tall, 1000:1, no vectors

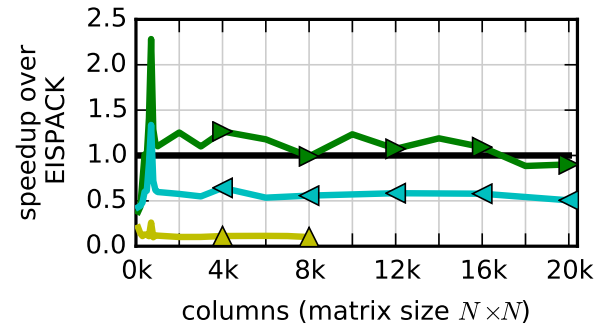

(b) square, with vectors

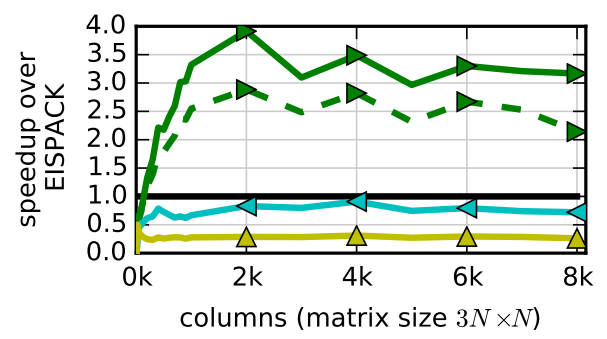

(d) tall, $3: 1$, with vectors

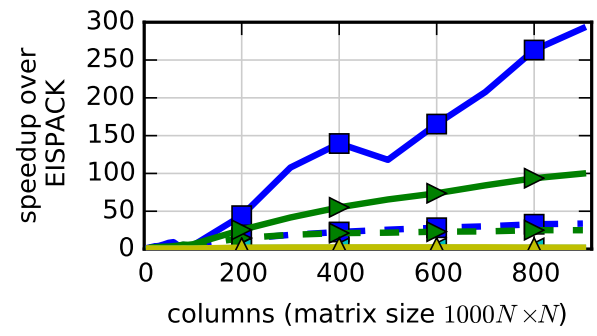

(f) tall, 1000:1, with vectors

\begin{tabular}{llll|}
$\triangle$ & $\triangle$ Eigen Jacobi & & $\square$ LAPACK Precond. Jacobi (1 core) \\
$\triangleleft$ & $\triangleleft$ LAPACK Jacobi & $\square$ & LAPACK QR iter. \\
& $\rightarrow$ LAPACK Precond. Jacobi & $-\square$ & LAPACK QR iter. (1 core) \\
\hline
\end{tabular}

Fig. 27 Comparison of LAPACK's one-sided Jacobi, preconditioned one-sided Jacobi, and Eigen's two-sided Jacobi.

both row-wise and column-wise, making for poor cache performance. For square matrices, it does not do any preconditioning. For tall matrices, it uses QRP, which improves its relative performance somewhat. (Note that Eigen can be configured to instead call LAPACK's QR iteration method.)

Figure 28 shows results for the preconditioned one-sided block Jacobi method. We tested two variants of the preconditioning, one using QRP, the other using regular QR factorization (no pivoting). In both cases, this was followed by an LQ factorization. This implementation has explicit parallelism via MPI. It uses ScaLAPACK for the QRP, QR, and LQ factorizations. We see that with QRP + LQ, it performed similarly to ScaLAPACK QR iteration, while with QR + LQ, it was a bit faster, matching LAPACK's QR iteration in performance for the tall, 3:1 case. 


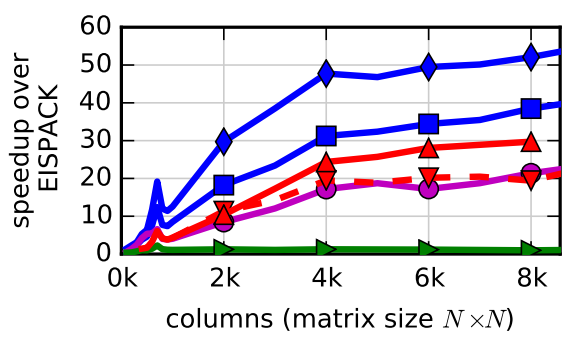

(a) square, with vectors

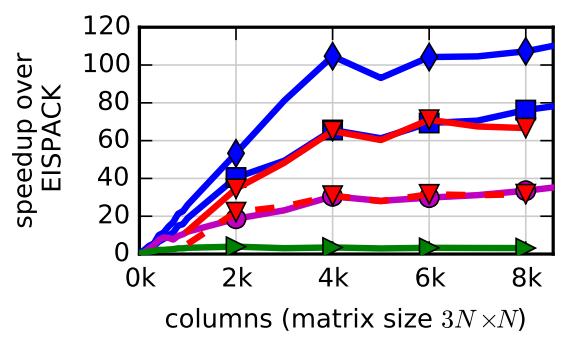

(b) tall, 3:1, with vectors

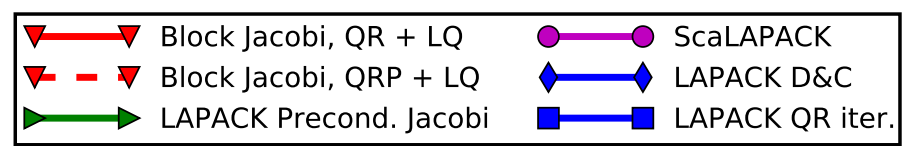

Fig. 28 Comparison of preconditioned one-side block Jacobi, LAPACK's preconditioned one-sided Jacobi, QR iteration, and DECC.

13. Accuracy. While Jacobi methods have struggled to compete with the performance of bidiagonalization methods, for some classes of matrices they have a distinct advantage in accuracy, which is now their main motivation. In this section, we briefly explore the accuracy differences between methods. The traditional perturbation theory [32] for both bidiagonalization and Jacobi methods shows that

$$
\frac{\left|\sigma_{i}-\hat{\sigma}_{i}\right|}{\sigma_{i}} \leq O(\epsilon) \kappa(A)
$$

where $\sigma_{i}$ and $\hat{\sigma}_{i}$ are the singular values of $A$ and $A+\delta A$, respectively, with a small perturbation $\delta A$ such that $\|\delta A\|_{2} \leq O(\epsilon)\|A\|_{2}$, and $\kappa(A)$ is the condition number of $A$. This implies that large singular values are computed accurately, but small singular values may be totally inaccurate if $\kappa(A)$ is large. For the one-sided Jacobi SVD method, this bound can be improved. Specifically, on matrices of the form $A=C D$, where $C$ has columns with unit two-norm and $D$ is diagonal, Demmel and Veselić [32] proved the bound

$$
\frac{\left|\sigma_{i}-\hat{\sigma}_{i}\right|}{\sigma_{i}} \leq O(\epsilon) \kappa(C)
$$

Crucially, it is possible that $\kappa(C) \ll \kappa(A)$, particularly in the instance of a strongly scaled matrix where $D$ is ill-conditioned. If ill-conditioning is artificial, due to poor scaling, then one-sided Jacobi will be unaffected by it and will compute even small singular values to high relative accuracy. Demmel et al. [30] extended methods of computing the SVD with high relative accuracy to a wider class of matrices of the form $A=X D Y^{T}$, where $D$ is diagonal and $X$ and $Y$ are well-conditioned.

Similar results apply for the two-sided Jacobi eigenvalue method with a positive definite matrix $A=D^{T} B D$ [32]. For eigenvalues of an indefinite matrix, though, QR iteration may be more accurate than Jacobi [108].

When applied to triangular matrices, Matejaš and Hari [88, 89] proved that the two-sided Jacobi SVD method also attains high relative accuracy. One can preprocess a general matrix using QRP to obtain such a triangular matrix. 


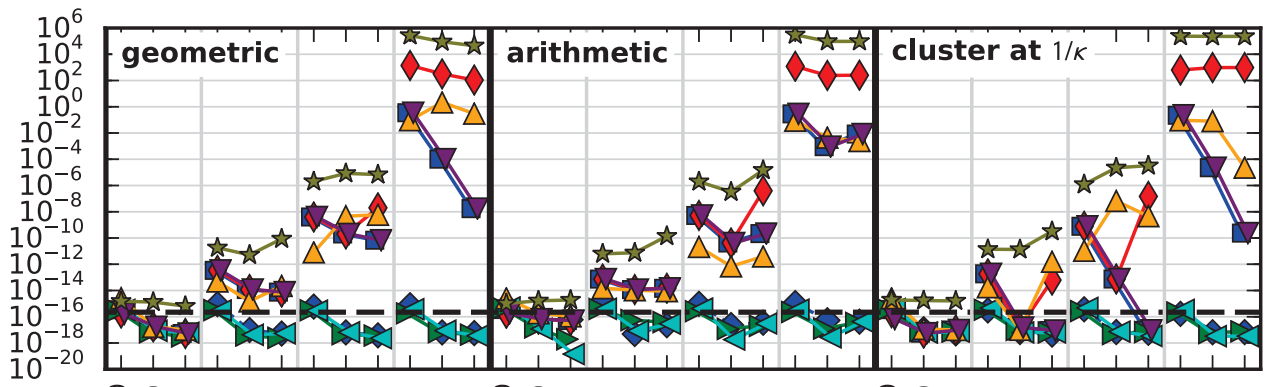

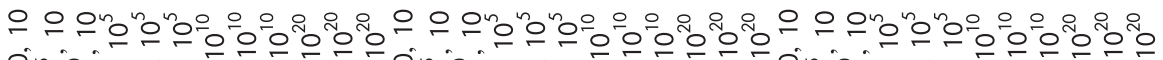

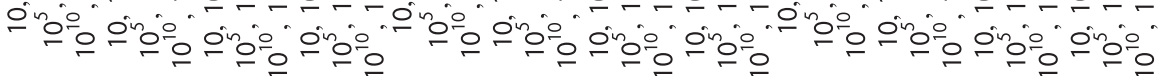

$\uparrow \uparrow^{-} \uparrow^{-} \quad k(C), k(D)$

\begin{tabular}{|c|c|}
\hline 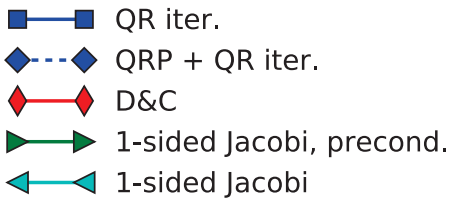 & $\begin{array}{l}\triangle \triangle \text { Eigen 2-sided Jacobi } \\
\square \text { Bisection } \\
\longleftarrow \text { MRRR } \\
--\varepsilon\end{array}$ \\
\hline
\end{tabular}

Fig. 29 Maximum relative error in singular values, $\max \left|\hat{\sigma}_{i}-\sigma_{i}\right| /\left(\kappa(C) \sigma_{i}\right)$, for $i=1, \ldots, 100$, with various test matrices. Figure 30 shows details for three instances indicated by arrows: geometric distribution with $(\kappa(C), \kappa(D))=\left(10^{5}, 10\right) ;\left(10^{5}, 10^{10}\right) ;\left(10^{5}, 10^{20}\right)$.

Applied to a bidiagonal matrix, the implicit zero-shift variant of QR iteration and the bisection method have been shown to achieve high relative accuracy for all singular values [31]. However, the classical reduction from dense to bidiagonal perturbs the singular values so the exact singular values of the bidiagonal matrix no longer have high relative accuracy for the original matrix $A$. Hence, any method based on an initial reduction to bidiagonal will lose relative accuracy for small singular values of an ill-conditioned matrix. To address this deficiency, Barlow [7] developed a more accurate bidiagonalization, using QRP followed by a Givens rotation-based bidiagonalization. Recently, Drmač [43] demonstrated that preprocessing a matrix with QRP (LAPACK's dgeqp3 routine) is sufficient to enable a subsequent QR iteration or bisection to have high relative accuracy (but not $\mathrm{D} \& \mathrm{C}$, which is not as accurate as QR iteration).

Here we test the accuracy of various methods on matrices with three different distributions of singular values: arithmetic, geometric, and a cluster at $1 / \kappa(C)$, as described in section 2. For each distribution, we generate singular values $\Sigma$ with condition number $\kappa(C)$, scale them so that $\sum \sigma_{i}^{2}=n$, and set $\tilde{C}=U \Sigma V^{T}$, where $U$ and $V$ are random orthogonal matrices from the Haar distribution [106]. To satisfy the conditions of (13), we use the method by Davies and Higham [26] to make $C=\tilde{C} W$ with columns of unit two-norm, where $W$ is orthogonal. Finally, we set $A=C D$, where $D$ is diagonal with entries whose logarithms are random uniform on $(\log (1 / \kappa(D)), \log (1))$. For each distribution, we set $n=100$ and vary $\kappa(C) \in\left\{10,10^{5}, 10^{10}\right\}$ and $\kappa(D) \in\left\{10,10^{5}, 10^{10}, 10^{20}\right\}$. For a reference solution, we used MATLAB's [90] variable-precision arithmetic (vpa) with 64 digits.

Figure 29 demonstrates the significant difference between one-sided Jacobi methods and bidiagonalization methods (QR iteration, D\&C, bisection, MRRR). Both 
one-sided Jacobi methods achieve high relative accuracy for all singular values, at or below the dashed line representing machine $\epsilon$. For small scaling, with $\kappa(D)=10$, all methods achieve high accuracy on all the matrices. Most of the bidiagonalization methods show increased relative errors as the scaling $\kappa(D)$ grows. For $\kappa(D)=10^{20}$, the maximum errors were sometimes larger than 1, i.e., no correct digits in the smallest singular values. Among bidiagonalization methods, the exception was preprocessing using QRP, then using QR iteration ( $\mathrm{QRP}+\mathrm{QR}$ iter., blue diamonds), which also achieved high relative accuracy, as predicted by Drmač.

QR iteration (blue squares) and bisection (purple down triangles) produce extremely similar errors, demonstrating that they both accurately compute singular values of the bidiagonal matrix, and the error occurs in the reduction to bidiagonal. Once the condition number $\kappa(A)$ exceeds $1 / \epsilon$, D\&C (red diamonds) has much worse error than QR iteration. Even with modest scaling, MRRR (stars) has the worst error. Eigen's two-sided Jacobi (orange up triangles) also exhibits significant error as the scaling increases. Preprocessing with QRP before Eigen's two-sided Jacobi (not shown) improved the accuracy, but not to the high relative accuracy of one-sided Jacobi. Based on [89], other two-sided Jacobi implementations are expected to achieve high relative accuracy.

To explain these results in more detail, we look at three specific cases for the geometric distribution with $\kappa(C)=10^{5}$ and $\kappa(D) \in\left\{10,10^{10}, 10^{20}\right\}$. In Figure 30, the left column shows the actual singular values, in both log and linear scale, while the right column shows the relative error in each singular value, $\sigma_{i}$, from $i=1, \ldots, 100$. In the top row, with minimal scaling $(\kappa(D)=10)$, all the methods achieve high accuracy, below $\epsilon$ in almost all cases. Eigen has a slightly higher error for large singular values, and MRRR is slightly higher for small singular values.

In the middle row, with modest scaling $\left(\kappa(D)=10^{10}\right)$, the one-sided Jacobi methods and QRP + QR iteration maintain high relative accuracy for all singular values. The bidiagonalization methods have high accuracy for the large singular values (near $\sigma_{1}$ ), but the relative error increases for small singular values, losing digits of accuracy. Eigen's error also increases.

In the bottom row, with large scaling $\left(\kappa(D)=10^{20}\right)$, the error of bidiagonalization methods for small singular values grows even more. As seen in the bottom-left graph, several methods compute singular values that diverge noticeably from the reference solution. For this matrix with $\sigma_{\max } \approx 10^{20}$, MRRR declares all $\sigma_{i}<10^{7}$ to be $3.27 \times 10^{7}$, i.e., it cannot resolve smaller singular values. Similarly, for D\&C, all $\sigma_{i}<10^{3}$ are computed as $6.91 \times 10^{3}$. Eigen also has issues for $\sigma<10^{4}$, though it does not flatline as MRRR and D\&C do. QR iteration and bisection follow the true singular values much more closely, but still exhibit significant error for small singular values.

14. Additional Test Cases. So far, we have mostly considered the performance of random uniform matrices. In this section, we look briefly at additional test cases using various distributions of singular values. Our purpose here is to give the reader an idea of the variability in performance and how representative the random uniform tests are. The distribution of singular values affects the performance of various SVD algorithms differently. For QR iteration and D\&C, whenever a singular value is determined with sufficient accuracy, it can be removed to shrink the problem size, a process known as deflation, improving the performance. For MRRR, having singular values close to one another will cause it to recurse further in the representation tree, decreasing its performance [119]. For one-sided Jacobi, matrices that are close to orthogonal-i.e., most of the weight of $A^{T} A$ is near the diagonal - converge faster. 

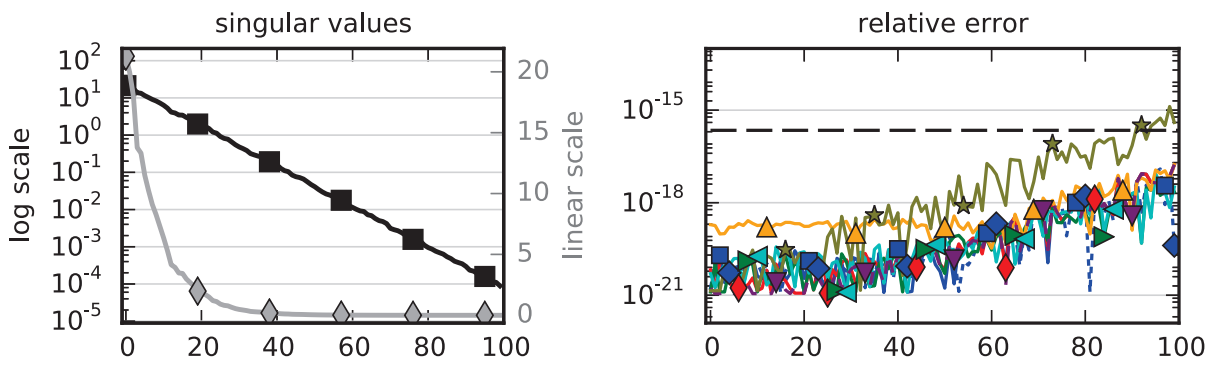

(a) minimal scaling, $\kappa(C)=10^{5}, \kappa(D)=10, \kappa(A)=2.4 \times 10^{5}$
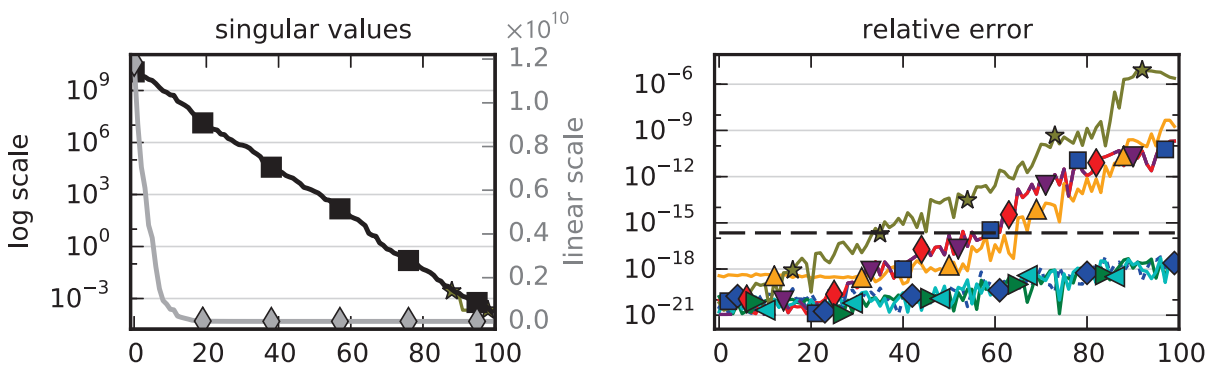

(b) moderate scaling, $\kappa(C)=10^{5}, \kappa(D)=10^{10}, \kappa(A)=6.9 \times 10^{13}$
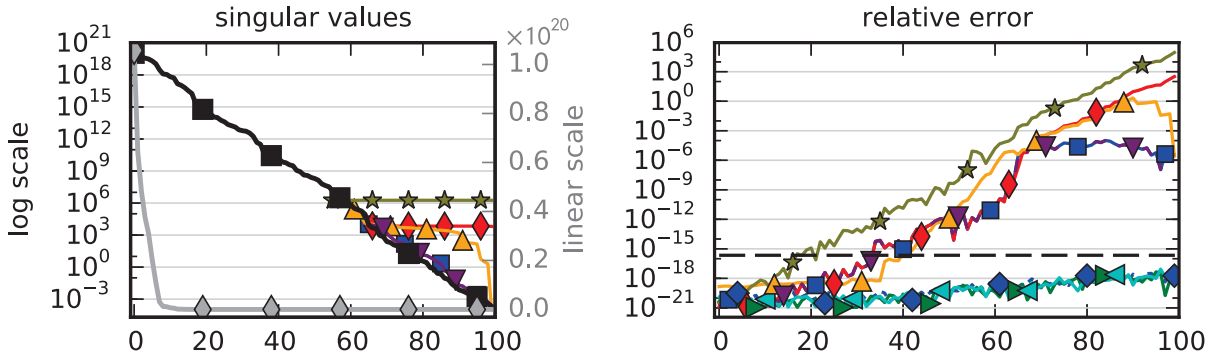

(c) large scaling, $\kappa(C)=10^{5}, \kappa(D)=10^{20}, \kappa(A)=5.2 \times 10^{23}$

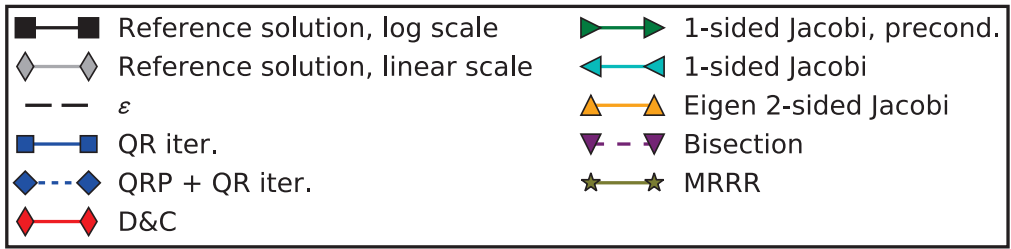

Fig. 30 Singular values of $A=C D$ are plotted twice in the left column, once in log scale (black squares) and once in linear scale (gray diamonds). In most cases, computed singular values are visually coincident with the reference solution (log scale). The right column shows relative error in each singular value, $\left|\hat{\sigma}_{i}-\sigma_{i}\right| /\left(\kappa(C) \sigma_{i}\right)$. The $x$ axis indexes the singular values from largest to smallest, $i=1, \ldots, 100$.

Figure 31 shows results for six methods on various matrices. These all use the LAPACK implementations, except MRRR which uses a modification of the bisection dgesvdx code, as described in section 9 . Note that the $y$-axis scale is different for QR iteration, D\&C, and MRRR compared to Jacobi and bisection. See section 2 


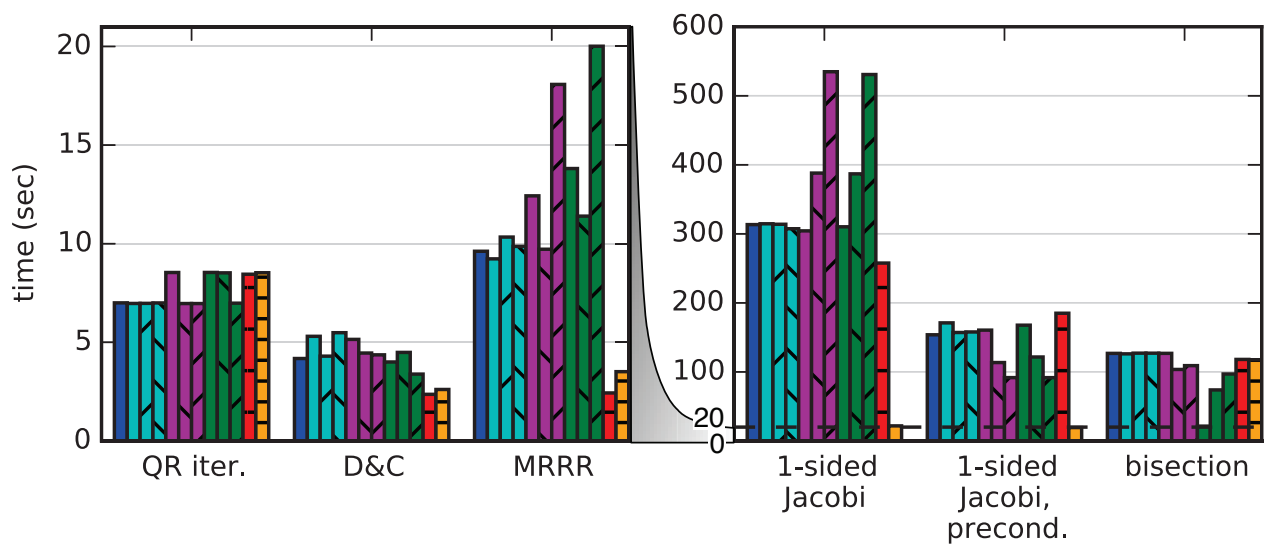

\begin{tabular}{|c|c|c|}
\hline entries random uniform & geometric, $\kappa=10$ & log-random, $\kappa=10^{5}$ \\
\hline arithmetic, $\kappa=10$ & geometric, $\kappa=10^{5}$ & log-random, $\kappa=10^{10}$ \\
\hline arithmetic, $\kappa=10^{5}$ & geometric, $\kappa=10^{10}$ & cluster at $1 / \kappa, \kappa=10^{5}$ \\
\hline arithmetic, $\kappa=10^{10}$ & log-random, $\kappa=10$ & cluster at $1, \kappa=10^{5}$ \\
\hline
\end{tabular}

Fig. 3I Time to compute the full $S V D$ for $n=3000$. Note the change in the $y$ axis; dashed line at $t=20$ corresponds to the $y$ axis in the left plot.

for a description of the matrix types. For each algorithm, the first, blue bar is for a random uniform matrix, matching most of the results elsewhere in this paper. The geometric and log-random distributions themselves are similar, so in most cases their performance trends are similar, except when using bisection. We see that for QR iteration, the performance for most matrices is similar to that of random uniform, with a few being up to $18 \%$ slower. For D\&C, the arithmetic distribution (cyan) was up to $24 \%$ slower, while log-random (green) was up to $23 \%$ faster than random uniform. The two clusters (red, orange) were $77 \%$ and $60 \%$ faster, due to significant deflation. MRRR is more variable, with geometric (purple) and log-random (green) being up to $47 \%$ and $52 \%$ slower on ill-conditioned matrices $\left(\kappa=10^{10}\right)$, while both clusters of repeated singular values were up to $3 \times$ faster than random uniform. Arithmetic (cyan) was not significantly affected by conditioning.

Because one-sided Jacobi and bisection were significantly slower, they are plotted with a different $y$ axis. In all cases, one-sided Jacobi and bisection were slower than QR iteration, D\&C, and MRRR. The geometric (purple) and log-random (green) matrices exhibited opposite behavior for the two Jacobi methods: for plain Jacobi, both matrices became slower as the conditioning worsened, while for preconditioned Jacobi, both became faster. A cluster at $1 / \kappa$ took similar time to random. A cluster at 1 was much faster, because $A^{T} A$ is already nearly diagonal, but preconditioning did not further improve it. Bisection was surprisingly $4.9 \times$ faster for a well-conditioned $(\kappa=10)$ log-random matrix, but the speedup decreased for poorer conditioning. As we saw earlier in section 8 when computing a subset of vectors, clusters were not advantageous. 
While the performance does vary for different classes of matrices - sometimes substantially - at a high level, our performance conclusions remain valid: D\&C is the fastest (being tied with MRRR in one case), then QR iteration, then MRRR. One-sided Jacobi is the slowest method, with preconditioning generally improving its speed, often by a factor of $2 \times$ or more. For computing all vectors, bisection is also slow; its main advantage is in computing a subset of vectors, as previously shown in section 8 .

15. Conclusions. As we have seen, algorithms to compute the SVD have continually evolved to address changes in computer hardware design, as well as advancements in mathematics. Early implementations such as EISPACK demonstrated that computing the SVD stably was feasible. Later implementations focused on improving the performance, first by using Level 1 BLAS for vector computers, then by reformulating the algorithm for Level 3 BLAS to address the emergence of cache-based memory hierarchies. More recently, a two-stage algorithm shifted even more operations from Level 2 to Level 3 BLAS. These changes have addressed the growing gap between memory bandwidth and computational speed, as well as enabling greater use of parallel hardware. Implementations have also taken advantage of different architectures such as distributed-memory computers and accelerators. Mathematical advancements have been important in reducing the number of operations performed. For tall-skinny problems, using an initial QR factorization can eliminate a quarter to half of the operations. For square matrices, the $\mathrm{D} \& \mathrm{C}$ algorithm reduces operations by nearly half. For Jacobi methods, preconditioning has been vital to improving convergence, while at the same time making computation of singular vectors more efficient. Block Jacobi methods with dynamic selection of subproblems have become competitive with some bidiagonalization methods. Improvements in algorithms used to preprocess a matrix, such as using a CAQR factorization [29] for tall-skinny matrices, or future improvements to QRP methods, are immediately applicable to benefitting SVD performance.

As we build the next generation of linear algebra software targeting exascale computers [77], the goal is to integrate these techniques - such as the two-stage reduction to bidiagonal, accelerators, and distributed computing - into a scalable SVD solver. While the techniques have been demonstrated to work, the challenge is being able to hide communication latencies in large distributed machines. Bottlenecks due to Amdahl's law, such as solving the bidiagonal SVD, will also be crucial to resolve. Improving algorithms to remove communication and memory bandwidth limitations becomes critically important.

For certain classes of matrices that are strong scaled, classical methods based on reduction to bidiagonal will not accurately compute small singular values. In these cases, one should turn to Jacobi methods or preprocessing the matrix using QRP to attain high relative accuracy.

We have focused on solving dense systems. There are, of course, different techniques for solving SVD problems with sparse linear systems. Also, if one is concerned with only an approximate, low rank solution, then using a randomized SVD algorithm [99] may be another avenue to pursue. This is often the case for huge systems arising from big data problems.

Here we have compared implementations on a common, modern architecture. To give some historical perspective, in 1977, EISPACK took 0.79 seconds (1.7 Mflop/s) to compute singular values for $n=80$ on an IBM 370/195 [105]. Today, the same EISPACK code achieves $0.74 \mathrm{Gflop} / \mathrm{s}$ on large problems, yielding over two ordersof-magnitude advancement in single-core hardware speed. On top of this, we have 
shown an additional two orders-of-magnitude improvement going from EISPACK to PLASMA (146 Gflop/s) on a multicore architecture, and four orders of magnitude to DPLASMA (6.8 Tflop/s) on a distributed-memory machine - while moving from solving systems of dimension 100 to over 100,000-yielding over six orders-of-magnitude performance improvement in 40 years.

Acknowledgments. We thank Martin Bečka, Gabriel Okša, and Marián Vajteršic for use of their block Jacobi code; Osni Marques for assistance with the MRRR code; and the anonymous reviewers for feedback to improve the quality and scope of this work. Furthermore, we would like to thank Intel for access to their distributed computing platform for testing ScaLAPACK and DPLASMA.

\section{REFERENCES}

[1] E. Agullo, B. Hadri, H. Ltaief, and J. Dongarrra, Comparative study of one-sided factorizations with multiple software packages on multi-core hardware, in Proceedings of the Conference on High Performance Computing, Networking, Storage and Analysis (SC'09), ACM, 2009, art. 20, https://doi.org/10.1145/1654059.1654080. (Cited on pp. 836, 838)

[2] E. Anderson, Z. Bai, C. Bischof, S. Blackford, J. Dongarra, J. Du Croz, A. GreenBaum, S. Hammarling, A. McKenney, and D. Sorensen, LAPACK Users' Guide, 3rd ed., SIAM, Philadelphia, 1999, https://doi.org/10.1137/1.9780898719604. (Cited on p. 814)

[3] H. Andrews and C. Patterson, Singular value decomposition (SVD) image coding, IEEE Trans. Commun., 24 (1976), pp. 425-432, https://doi.org/10.1109/TCOM.1976.1093309. (Cited on p. 810)

[4] P. Arbenz and G. H. Golub, On the spectral decomposition of Hermitian matrices modified by low rank perturbations with applications, SIAM J. Matrix Anal. Appl., 9 (1988), pp. 4058, https://doi.org/10.1137/0609004. (Cited on p. 825)

[5] P. ARbenz AND I. SlapničAR, An analysis of parallel implementations of the block-Jacobi algorithm for computing the SVD, in Proceedings of the 17th International Conference on Information Technology Interfaces ITI, 1995, pp. 13-16, http://citeseerx.ist.psu.edu/ viewdoc/summary?doi=10.1.1.53.4595. (Cited on p. 850)

[6] G. Ballard, J. Demmel, and N. Knight, Avoiding communication in successive band reduction, ACM Trans. Parallel Comput., 1 (2015), p. 11, https://doi.org/10.1145/2686877. (Cited on p. 835)

[7] J. L. BARLOW, More accurate bidiagonal reduction for computing the singular value decomposition, SIAM J. Matrix Anal. Appl., 23 (2002), pp. 761-798, https://doi.org/10.1137/ S0895479898343541. (Cited on p. 854)

[8] M. BEČKA, G. OKŠA, AND M. VAJTERŠIC, Dynamic ordering for a parallel block-Jacobi SVD algorithm, Parallel Comput., 28 (2002), pp. 243-262, https://doi.org/10.1016/ S0167-8191(01)00138-7. (Cited on pp. 846, 850)

[9] M. BEČKA, G. OKŠA, AND M. VAJTERŠIC, New dynamic orderings for the parallel one-sided block-Jacobi SVD algorithm, Parallel Process. Lett., 25 (2015), art. 1550003, https://doi. org/10.1142/S0129626415500036. (Cited on pp. 849, 850)

[10] M. BeČKA, G. OKŠA, M. VAJTERŠIC, AND L. GRIGORI, On iterative $Q R$ pre-processing in the parallel block-Jacobi SVD algorithm, Parallel Comput., 36 (2010), pp. 297-307, https: //doi.org/10.1016/j.parco.2009.12.013. (Cited on p. 849)

[11] M. BEČKA AND M. VAJTERŠIC, Block-Jacobi SVD algorithms for distributed memory systems I: Hypercubes and rings, Parallel Algorithms Appl., 13 (1999), pp. 265-287, https://doi. org/10.1080/10637199808947377. (Cited on p. 847)

[12] M. BEČKA AND M. VAJTERŠIC, Block-Jacobi SVD algorithms for distributed memory systems II: Meshes, Parallel Algorithms Appl., 14 (1999), pp. 37-56, https://doi.org/10.1080/ 10637199808947370. (Cited on p. 847)

[13] C. Bischof, B. Lang, and X. Sun, Algorithm 807: The SBR Toolbox-software for successive band reduction, ACM Trans. Math. Software, 26 (2000), pp. 602-616, https://doi.org/10. 1145/365723.365736. (Cited on p. 835)

[14] C. Bischof AND C. VAN LOAN, The WY representation for products of Householder matrices, SIAM J. Sci. Statist. Comput., 8 (1987), pp. 2-13, https://doi.org/10.1137/0908009. (Cited on p. 814)

Copyright $@$ by SIAM. Unauthorized reproduction of this article is prohibited. 
[15] C. H. Bischof, Computing the singular value decomposition on a distributed system of vector processors, Parallel Comput., 11 (1989), pp. 171-186, https://doi.org/10.1016/ 0167-8191(89)90027-6. (Cited on pp. 847, 849, 850)

[16] L. S. Blackford, J. Choi, A. Cleary, E. D’Azevedo, J. Demmel, I. Dhillon, J. Dongarra, S. Hammarling, G. Henry, A. Petitet et al., ScalaPACK Users' Guide, SIAM, Philadelphia, 1997, https://doi.org/10.1137/1.9780898719642. (Cited on p. 822)

[17] L. S. Blackford, A. Petitet, R. Pozo, K. Remington, R. C. Whaley, J. Demmel, J. Dongarra, I. Duff, S. Hammarling, G. Henry et Al., An updated set of basic linear algebra subprograms (BLAS), ACM Trans. Math. Software, 28 (2002), pp. 135151, https://doi.org/10.1145/567806.567807. (Cited on p. 820)

[18] G. Bosilca, A. Bouteiller, A. Danalis, M. Faverge, A. Haidar, T. Herault, J. Kurzak, J. Langou, P. Lemarinier, H. Ltaief, et Al., Flexible development of dense linear algebra algorithms on massively parallel architectures with DPLASMA, in 2011 IEEE International Symposium on Parallel and Distributed Processing Workshops and Ph.D. Forum (IPDPSW), IEEE, 2011, pp. 1432-1441, https://doi.org/10.1109/IPDPS.2011.299. (Cited on pp. 835, 841)

[19] G. Bosilca, A. Bouteiller, A. Danalis, T. Herault, P. Lemarinier, and J. Dongarra, DAGuE: A generic distributed DAG engine for high performance computing, Parallel Comput., 38 (2012), pp. 37-51, https://doi.org/10.1016/j.parco.2011.10.003. (Cited on pp. 835,841$)$

[20] W. H. Boukaram, G. Turkiyyah, H. Ltaief, and D. E. Keyes, Batched QR and SVD algorithms on GPUs with applications in hierarchical matrix compression, Parallel Comput., 74 (2017), pp. 19-33, https://doi.org/10.1016/j.parco.2017.09.001. (Cited on p. 850)

[21] R. P. BREnT AND F. T. LUK, The solution of singular-value and symmetric eigenvalue problems on multiprocessor arrays, SIAM J. Sci. Statist. Comput., 6 (1985), pp. 69-84, https://doi.org/10.1137/0906007. (Cited on p. 847)

[22] R. P. BRent, F. T. LUK, AND C. VAN LOAN, Computation of the singular value decomposition using mesh-connected processors, J. VLSI Comput. Syst., 1 (1985), pp. 242-270, http: //maths-people.anu.edu.au/ brent/pd/rpb080i.pdf. (Cited on pp. 844, 846, 847)

[23] T. F. ChAn, An improved algorithm for computing the singular value decomposition, ACM Trans. Math. Software, 8 (1982), pp. 72-83, https://doi.org/10.1145/355984.355990. (Cited on pp. 813, 818)

[24] J. Choi, J. Dongarra, and D. W. Walker, The design of a parallel dense linear algebra software library: Reduction to Hessenberg, tridiagonal, and bidiagonal form, Numer. Algorithms, 10 (1995), pp. 379-399, https://doi.org/10.1007/BF02140776. (Cited on p. 822)

[25] J. J. M. CUppen, A divide and conquer method for the symmetric tridiagonal eigenproblem, Numer. Math., 36 (1980), pp. 177-195, https://doi.org/10.1007/BF01396757. (Cited on p. 825)

[26] P. I. Davies and N. J. Higham, Numerically stable generation of correlation matrices and their factors, BIT, 40 (2000), pp. 640-651, https://doi.org/10.1023/A:102238421. (Cited on p. 854)

[27] P. P. M. DE RIJK, A one-sided Jacobi algorithm for computing the singular value decomposition on a vector computer, SIAM J. Sci. Statist. Comput., 10 (1989), pp. 359-371, https://doi.org/10.1137/0910023. (Cited on pp. 846, 847)

[28] S. Deerwester, S. T. Dumais, G. W. Furnas, T. K. Landauer, and R. Harshman, Indexing by latent semantic analysis, J. Amer. Soc. Inform. Sci., 41 (1990), pp. 391407, https://doi.org/10.1002/(SICI)1097-4571(199009)41:6<391::AID-ASI1>3.0.CO;2-9. (Cited on p. 810)

[29] J. Demmel, L. Grigori, M. Hoemmen, and J. Langou, Communication-optimal parallel and sequential $Q R$ and LU factorizations, SIAM J. Sci. Comput., 34 (2012), pp. A206-A239, https://doi.org/10.1137/080731992. (Cited on pp. 838, 858)

[30] J. Demmel, M. Gu, S. Eisenstat, I. Slapničar, K. Veselić, and Z. Drmač, Computing the singular value decomposition with high relative accuracy, Linear Algebra Appl., 299 (1999), pp. 21-80, https://doi.org/10.1016/S0024-3795(99)00134-2. (Cited on p. 853)

[31] J. Demmel and W. Kahan, Accurate singular values of bidiagonal matrices, SIAM J. Sci. Statist. Comput., 11 (1990), pp. 873-912, https://doi.org/10.1137/0911052. (Cited on pp. $817,831,854)$

[32] J. Demmel And K. Veselić, Jacobi's method is more accurate than QR, SIAM J. Matrix Anal. Appl., 13 (1992), pp. 1204-1245, https://doi.org/10.1137/0613074. (Cited on pp. 845, $853)$

Copyright (c) by SIAM. Unauthorized reproduction of this article is prohibited. 
[33] J. W. Demmel, Applied Numerical Linear Algebra, SIAM, Philadelphia, 1997, https://doi. org/10.1137/1.9781611971446. (Cited on p. 829)

[34] J. W. Demmel, I. Dhillon, and H. Ren, On the correctness of some bisection-like parallel eigenvalue algorithms in floating point arithmetic, Electron. Trans. Numer. Anal., 3 (1995), pp. 116-149, http://emis.ams.org/journals/ETNA/vol.3.1995/pp116-149.dir/ pp116-149.pdf. (Cited on p. 828)

[35] I. S. Dhillon, A New $O\left(n^{2}\right)$ Algorithm for the Symmetric Tridiagonal Eigenvalue/Eigenvector Problem, Ph.D. thesis, EECS Department, University of California, Berkeley, 1997, http://www.dtic.mil/docs/citations/ADA637073. (Cited on p. 829)

[36] I. S. Dhillon AND B. N. PARlett, Multiple representations to compute orthogonal eigenvectors of symmetric tridiagonal matrices, Linear Algebra Appl., 387 (2004), pp. 1-28, https://doi.org/10.1016/j.laa.2003.12.028. (Cited on pp. 829, 832)

[37] I. S. Dhillon And B. N. PARLetT, Orthogonal eigenvectors and relative gaps, SIAM J. Matrix Anal. Appl., 25 (2004), pp. 858-899, https://doi.org/10.1137/S0895479800370111. (Cited on pp. 831, 832)

[38] I. S. Dhillon, B. N. Parlett, And C. Vömel, The design and implementation of the MRRR algorithm, ACM Trans. Math. Software, 32 (2006), pp. 533-560, https://doi.org/10.1145/ 1186785.1186788. (Cited on pp. 829, 831)

[39] J. Dongarra, J. R. Bunch, C. B. Moler, and G. W. Stewart, LiNPACK Users' Guide, SIAM, Philadelphia, 1979, https://doi.org/10.1137/1.9781611971811. (Cited on p. 814)

[40] J. Dongarra, J. Du Croz, S. Hammarling, and I. S. Duff, A set of level 3 basic linear algebra subprograms, ACM Trans. Math. Software, 16 (1990), pp. 1-17, https://doi.org/ 10.1145/77626.79170. (Cited on p. 814)

[41] J. Dongarra, J. Du Croz, S. Hammarling, and R. J. Hanson, An extended set of FORTRAN basic linear algebra subprograms, ACM Trans. Math. Software, 14 (1988), pp. 1-17, https://doi.org/10.1145/42288.42291. (Cited on p. 814)

[42] J. Dongarra, D. C. Sorensen, and S. J. Hammarling, Block reduction of matrices to condensed forms for eigenvalue computations, J. Comput. Appl. Math., 27 (1989), pp. 215227, https://doi.org/10.1016/0377-0427(89)90367-1. (Cited on p. 816)

[43] Z. Drmač, Algorithm 977: A QR-preconditioned QR SVD method for computing the SVD with high accuracy, ACM Trans. Math. Software, 44 (2017), p. 11, https://doi.org/10. 1145/3061709. (Cited on p. 854)

[44] Z. DrmaČ And K. Veselić, New fast and accurate Jacobi SVD algorithm, I, SIAM J. Matrix Anal. Appl., 29 (2008), pp. 1322-1342, https://doi.org/10.1137/050639193. (Cited on pp. 848,850$)$

[45] Z. DrmaČ And K. Veselić, New fast and accurate Jacobi SVD algorithm, II, SIAM J. Matrix Anal. Appl., 29 (2008), pp. 1343-1362, https://doi.org/10.1137/05063920X. (Cited on pp. 849, 850)

[46] P. EBERLEIN, On one-sided Jacobi methods for parallel computation, SIAM J. Algebraic Discrete Methods, 8 (1987), pp. 790-796, https://doi.org/10.1137/0608064. (Cited on p. 847)

[47] Eigen, Eigen 3.3.3, 2017, http://eigen.tuxfamily.org/. (Cited on p. 850)

[48] K. V. Fernando And B. N. PARlett, Accurate singular values and differential qd algorithms, Numer. Math., 67 (1994), pp. 191-229, https://doi.org/10.1007/s002110050024. (Cited on p. 817)

[49] G. E. Forsythe And P. Henrici, The cyclic Jacobi method for computing the principal values of a complex matrix, Trans. Amer. Math. Soc., 94 (1960), pp. 1-23, https://doi.org/10. 2307/1993275. (Cited on pp. 844, 846)

[50] B. S. Garbow, J. M. Boyle, C. B. Moler, and J. Dongarra, Matrix eigensystem routines - EISPACK guide extension, Lecture Notes in Comput. Sci. 51, Springer, Berlin, 1977, https://doi.org/10.1007/3-540-08254-9. (Cited on p. 812)

[51] M. Gates, S. Tomov, and J. DongarraA, Accelerating the SVD two stage bidiagonal reduction and divide and conquer using GPUs, Parallel Comput., 74 (2018), pp. 3-18, https://doi.org/10.1016/j.parco.2017.10.004. (Cited on pp. 834, 835)

[52] G. Golub, Some modified matrix eigenvalue problems, SIAM Rev., 15 (1973), pp. 318-334, https://doi.org/10.1137/1015032. (Cited on p. 825)

[53] G. Golub And W. Kahan, Calculating the singular values and pseudo-inverse of a matrix, J. Soc. Indust. Appl. Math. Ser. B Numer. Anal., 2 (1965), pp. 205-224, https://doi.org/ 10.1137/0702016. (Cited on pp. 810, 811, 827)

[54] G. Golub And C. Reinsch, Singular value decomposition and least squares solutions, Numer. Math., 14 (1970), pp. 403-420, https://doi.org/10.1007/BF02163027. (Cited on p. 811)

[55] B. Grosser and B. Lang, Efficient parallel reduction to bidiagonal form, Parallel Comput., 25 (1999), pp. 969-986, https://doi.org/10.1016/S0167-8191(99)00041-1. (Cited on p. 835)

Copyright (C) by SIAM. Unauthorized reproduction of this article is prohibited. 
[56] M. Gu, J. Demmel, And I. Dhillon, Efficient Computation of the Singular Value Decomposition with Applications to Least Squares Problems, Tech. Report LBL-36201, Lawrence Berkeley Laboratory, 1994, http://www.cs.utexas.edu/users/inderjit/public_ papers/least_squares.pdf. (Cited on pp. 824, 825)

[57] M. Gu and S. C. Eisenstat, A Divide and Conquer Algorithm for the Bidiagonal SVD, Tech. Report YALEU/DCS/TR-933, Department of Computer Science, Yale University, 1992, http://cpsc.yale.edu/research/technical-reports/1992-technical-reports. (Cited on pp. 824, 825)

[58] M. Gu And S. C. Eisenstat, A stable and efficient algorithm for the rank-one modification of the symmetric eigenproblem, SIAM J. Matrix Anal. Appl., 15 (1994), pp. 1266-1276, https://doi.org/10.1137/S089547989223924X. (Cited on p. 825)

[59] M. Gu AND S. C. EISEnstAT, A divide-and-conquer algorithm for the bidiagonal SVD, SIAM J. Matrix Anal. Appl., 16 (1995), pp. 79-92, https://doi.org/10.1137/S0895479892242232. (Cited on pp. 824, 826)

[60] A. Haidar, J. Kurzak, AND P. LuszczeK, An improved parallel singular value algorithm and its implementation for multicore hardware, in Proceedings of the International Conference on High Performance Computing, Networking, Storage and Analysis (SC'13), ACM, 2013, art. 90, https://doi.org/10.1145/2503210.2503292. (Cited on pp. 835, 836, 837)

[61] A. Haidar, H. Ltaief, and J. Dongarra, Parallel reduction to condensed forms for symmetric eigenvalue problems using aggregated fine-grained and memory-aware kernels, in Proceedings of 2011 International Conference for High Performance Computing, Networking, Storage and Analysis (SC'11), ACM, 2011, art. 8, https://doi.org/10.1145/2063384. 2063394. (Cited on pp. 835, 837)

[62] A. Haidar, H. Ltaief, P. Luszczek, and J. Dongarra, A comprehensive study of task coalescing for selecting parallelism granularity in a two-stage bidiagonal reduction, in 2012 IEEE 26th International Parallel and Distributed Processing Symposium (IPDPS), IEEE, 2012, pp. 25-35, https://doi.org/10.1109/IPDPS.2012.13. (Cited on p. 835)

[63] S. Hammarling, A note on modifications to the Givens plane rotation, IMA J. Appl. Math., 13 (1974), pp. 215-218, https://doi.org/10.1093/imamat/13.2.215. (Cited on p. 851)

[64] V. HARI, Accelerating the SVD block-Jacobi method, Computing, 75 (2005), pp. 27-53, https: //doi.org/10.1007/s00607-004-0113-z. (Cited on pp. 849, 850)

[65] V. Hari And J. Matejaš, Accuracy of two SVD algorithms for $2 \times 2$ triangular matrices, Appl. Math. Comput., 210 (2009), pp. 232-257, https://doi.org/10.1016/j.amc.2008.12. 086. (Cited on pp. 846, 849)

[66] V. HARI AND K. VeSElić, On Jacobi methods for singular value decompositions, SIAM J. Sci. Statist. Comput., 8 (1987), pp. 741-754, https://doi.org/10.1137/0908064. (Cited on p. 846)

[67] M. Heath, A. Laub, C. Paige, and R. Ward, Computing the singular value decomposition of a product of two matrices, SIAM J. Sci. Statist. Comput., 7 (1986), pp. 1147-1159, https://doi.org/10.1137/0907078. (Cited on pp. 845, 846)

[68] M. R. Hestenes, Inversion of matrices by biorthogonalization and related results, J. Soc. Indust. Appl. Math., 6 (1958), pp. 51-90, https://doi.org/10.1137/0106005. (Cited on pp. 811,845$)$

[69] G. W. Howell, J. W. Demmel, C. T. Fulton, S. Hammarling, and K. Marmol, Cache efficient bidiagonalization using BLAS 2.5 operators, ACM Trans. Math. Software, 34 (2008), art. 14, https://doi.org/10.1145/1356052.1356055. (Cited on pp. 820, 822, 837)

[70] IBM CORPORATIOn, ESSL Guide and Reference, 2016, http://publib.boulder.ibm.com/ epubs/pdf/a2322688.pdf. (Cited on p. 814)

[71] Intel Corporation, User's Guide for Intel Math Kernel Library for Linux OS, 2015, http: //software.intel.com/en-us/mkl-for-linux-userguide. (Cited on pp. 812, 814)

[72] I. C. F. IPSEN, Computing an eigenvector with inverse iteration, SIAM Rev., 39 (2006), pp. 254-291, https://doi.org/10.1137/S0036144596300773. (Cited on pp. 829, 831)

[73] C. G. J. JACOBI, Über ein leichtes verfahren die in der theorie der säcularstörungen vorkommenden gleichungen numerisch aufzulösen, J. Reine Angew. Math., 30 (1846), pp. 51-94, http://eudml.org/doc/147275. (Cited on p. 843)

[74] E. Jessup And D. Sorensen, A divide and conquer algorithm for computing the singular value decomposition, in Proceedings of the Third SIAM Conference on Parallel Processing for Scientific Computing, 1989, SIAM, Philadelphia, pp. 61-66. (Cited on p. 825)

[75] W. Kahan, Accurate Eigenvalues of a Symmetric Tri-diagonal Matrix, Tech. Report, Stanford University, Stanford, CA, 1966, http://www.dtic.mil/docs/citations/AD0638796. (Cited on p. 828)

Copyright $@$ by SIAM. Unauthorized reproduction of this article is prohibited. 
[76] E. Kogbetliantz, Solution of linear equations by diagonalization of coefficients matrix, Quart. Appl. Math., 13 (1955), pp. 123-132, http://www.ams.org/journals/qam/ 1955-13-02/S0033-569X-1955-88795-9/S0033-569X-1955-88795-9.pdf. (Cited on pp. 811, 844)

[77] J. Kurzak, P. Wu, M. Gates, I. Yamazaki, P. Luszczek, G. Ragghianti, and J. DonGARRA, Designing SLATE: Software for Linear Algebra Targeting Exascale, SLATE Working Note 3, Innovative Computing Laboratory, University of Tennessee, 2017, http://www.icl.utk.edu/publications/swan-003. (Cited on p. 858)

[78] B. Lang, Parallel reduction of banded matrices to bidiagonal form, Parallel Comput., 22 (1996), pp. 1-18, https://doi.org/10.1016/0167-8191(95)00064-X. (Cited on p. 835)

[79] C. L. Lawson, R. J. Hanson, D. R. Kincaid, and F. T. Krogh, Basic linear algebra subprograms for FORTRAN usage, ACM Trans. Math. Software, 5 (1979), pp. 308-323, https://doi.org/10.1145/355841.355847. (Cited on p. 814)

[80] R.-C. LI, Solving Secular Equations Stably and Efficiently, Tech. Report UCB//CSD-94-851, Computer Science Division, University of California Berkeley, 1994, http://www.netlib. org/lapack/lawns/. Also: LAPACK Working Note 89. (Cited on pp. 825, 826)

[81] S. Li, M. Gu, L. Cheng, X. ChI, And M. Sun, An accelerated divide-and-conquer algorithm for the bidiagonal SVD problem, SIAM J. Matrix Anal. Appl., 35 (2014), pp. 1038-1057, https://doi.org/10.1137/130945995. (Cited on p. 826)

[82] H. Ltaief, J. Kurzak, and J. Dongarra, Parallel two-sided matrix reduction to band bidiagonal form on multicore architectures, IEEE Trans. Parallel Distrib. Syst., 21 (2010), pp. 417-423, https://doi.org/10.1109/TPDS.2009.79. (Cited on p. 835)

[83] H. Ltaief, P. LuszczeK, and J. Dongarra, High-performance bidiagonal reduction using tile algorithms on homogeneous multicore architectures, ACM Trans. Math. Software, 39 (2013), art. 16, https://doi.org/10.1145/2450153.2450154. (Cited on p. 835)

[84] F. T. LUK, Computing the singular-value decomposition on the ILLIAC IV, ACM Trans. Math. Software, 6 (1980), pp. 524-539, https://doi.org/10.1145/355921.355925. (Cited on pp. 845,850 )

[85] F. T. LuK and H. Park, On parallel Jacobi orderings, SIAM J. Sci. Statist. Comput., 10 (1989), pp. 18-26, https://doi.org/10.1137/0910002. (Cited on p. 847)

[86] O. Marques and P. B. VAsconcelos, Computing the bidiagonal SVD through an associated tridiagonal eigenproblem, in International Conference on Vector and Parallel Processing (VECPAR), Springer, 2016, pp. 64-74, https://doi.org/10.1007/978-3-319-61982-8_8. (Cited on pp. 827, 828, 832)

[87] W. F. Mascarenhas, On the convergence of the Jacobi method for arbitrary orderings, SIAM J. Matrix Anal. Appl., 16 (1995), pp. 1197-1209, https://doi.org/10.1137/ S0895479890179631. (Cited on p. 849)

[88] J. Matejaš And V. HaRI, Accuracy of the Kogbetliantz method for scaled diagonally dominant triangular matrices, Appl. Math. Comput., 217 (2010), pp. 3726-3746, https://doi.org/ 10.1016/j.amc.2010.09.020. (Cited on pp. 849, 853)

[89] J. Matejaš And V. HARI, On high relative accuracy of the Kogbetliantz method, Linear Algebra Appl., 464 (2015), pp. 100-129, https://doi.org/10.1016/j.laa.2014.02.024. (Cited on pp. $849,850,853,855)$

[90] MathWorks, MATLAB, 2017, http://www.mathworks.com/products/matlab.html. (Cited on p. 854 )

[91] J. D. MCCALPIN, A survey of memory bandwidth and machine balance in current high performance computers, IEEE Comput. Soc. Tech. Committee Comput. Architect. (TCCA) Newslett., 19 (1995), pp. 19-25, http://tab.computer.org/tcca/NEWS/DEC95/ dec95_mccalpin.ps. (Cited on p. 812)

[92] B. Moore, Principal component analysis in linear systems: Controllability, observability, and model reduction, IEEE Trans. Automat. Control, 26 (1981), pp. 17-32, https://doi. org/10.1109/TAC.1981.1102568. (Cited on p. 810)

[93] MPI Forum, MPI: A Message-Passing Interface Standard, Version 3.1, June 2015, http: //www.mpi-forum.org/. (Cited on p. 822)

[94] NVIDIA Corporation, CUDA Toolkit 7.0, March 2015, http://developer.nvidia.com/ cuda-zone. (Cited on p. 812)

[95] G. OKŠA AND M. VAJTERŠIC, Efficient pre-processing in the parallel block-Jacobi SVD algorithm, Parallel Comput., 32 (2006), pp. 166-176, https://doi.org/10.1016/j.parco.2005. 06.006. (Cited on p. 849)

[96] OpenBLAS, OpenBLAS User Manual, 2016, http://www.openblas.net/. (Cited on p. 814)

[97] B. N. Parlett, The new QD algorithms, Acta Numer., 4 (1995), pp. 459-491, https://doi. org/10.1017/S0962492900002580. (Cited on p. 817)

Copyright (c) by SIAM. Unauthorized reproduction of this article is prohibited. 
[98] B. N. Parlett and I. S. Dhillon, Fernando's solution to Wilkinson's problem: An application of double factorization, Linear Algebra Appl., 267 (1997), pp. 247-279, https: //doi.org/10.1016/S0024-3795(97)80053-5. (Cited on p. 832)

[99] V. Rokhlin, A. Szlam, And M. Tygert, A randomized algorithm for principal component analysis, SIAM J. Matrix Anal. Appl., 31 (2009), pp. 1100-1124, https://doi.org/10.1137/ 080736417. (Cited on p. 858)

[100] H. Rutishauser, Der quotienten-differenzen-algorithmus, Z. Angew. Math. Phys., 5 (1954), pp. 233-251, https://doi.org/10.1007/BF01600331. (Cited on p. 817)

[101] H. Rutishauser, Solution of eigenvalue problems with the LR-transformation, Nat. Bur. Standards Appl. Math. Ser., 49 (1958), pp. 47-81. (Cited on p. 817)

[102] H. Rutishauser, The Jacobi method for real symmetric matrices, in Handbook for Automatic Computation: Volume II: Linear Algebra, Grundlehren Math. Wiss. 186, Springer-Verlag, New York, 1971, pp. 202-211, https://doi.org/10.1007/978-3-642-86940-2. (Cited on pp. 844, 846)

[103] A. H. SAmeh, On Jacobi and Jacobi-like algorithms for a parallel computer, Math. Comp., 25 (1971), pp. 579-590, https://doi.org/10.1090/S0025-5718-1971-0297131-6. (Cited on p. 849$)$

[104] R. Schreiber ANd C. VAN LOAN, A storage-efficient WY representation for products of Householder transformations, SIAM J. Sci. Statist. Comput., 10 (1989), pp. 53-57, https: //doi.org/10.1137/0910005. (Cited on p. 814)

[105] B. T. Smith, J. M. Boyle, J. Dongarra, B. S. Garbow, Y. Ikebe, V. C. Klema, and C. B. Moler, Matrix Eigensystem Routines - EISPACK Guide, Second Edition, Lecture Notes in Comput. Sci. 6, Springer, Berlin, 1976, https://doi.org/10.1007/3-540-07546-1. (Cited on p. 858)

[106] G. W. STEWART, The efficient generation of random orthogonal matrices with an application to condition estimators, SIAM J. Numer. Anal., 17 (1980), pp. 403-409, https://doi.org/ 10.1137/0717034. (Cited on pp. 812, 854)

[107] G. W. SteWART, On the early history of the singular value decomposition, SIAM Rev., 35 (1993), pp. 551-566, https://doi.org/10.1137/1035134. (Cited on p. 810)

[108] G. W. Stewart, QR Sometimes Beats Jacobi, Tech. Report CS-TR-3434, University of Maryland, 1995, http://drum.lib.umd.edu/handle/1903/709. (Cited on p. 853)

[109] S. Tomov, R. NATH, And J. Dongarra, Accelerating the reduction to upper Hessenberg, tridiagonal, and bidiagonal forms through hybrid GPU-based computing, Parallel Comput., 36 (2010), pp. 645-654, https://doi.org/10.1016/j.parco.2010.06.001. (Cited on p. 833)

[110] S. Tomov, R. Nath, H. Ltaief, and J. Dongarra, Dense linear algebra solvers for multicore with GPU accelerators, in 2010 IEEE International Symposium on Parallel and Distributed Processing, Workshops and Phd Forum (IPDPSW), IEEE, 2010, pp. 1-8, https://doi.org/10.1109/IPDPSW.2010.5470941. (Cited on p. 834)

[111] M. A. Turk and A. P. Pentland, Face recognition using eigenfaces, in Proceedings of 1991 IEEE Computer Society Conference on Computer Vision and Pattern Recognition, IEEE, 1991, pp. 586-591, https://doi.org/10.1109/CVPR.1991.139758. (Cited on p. 810)

[112] C. VAN LOAN, The Block Jacobi Method for Computing the Singular Value Decomposition, Tech. Report TR 85-680, Cornell University, 1985, https://ecommons.cornell.edu/handle/ 1813/6520. (Cited on p. 849)

[113] F. G. Van Zee, R. A. Van de Geijn, and G. Quintana-Ortí, Restructuring the tridiagonal and bidiagonal QR algorithms for performance, ACM Trans. Math. Software, 40 (2014), p. 18, https://doi.org/10.1145/2535371. (Cited on p. 818)

[114] F. G. Van Zee, R. A. Van De Geijn, G. Quintana-Ortí, and G. J. Elizondo, Families of algorithms for reducing a matrix to condensed form, ACM Trans. Math. Software, 39 (2012), art. 2, https://doi.org/10.1145/2382585.2382587. (Cited on p. 822)

[115] R. C. Whaley and J. Dongarra, Automatically tuned linear algebra software, in Proceedings of the 1998 ACM/IEEE Conference on Supercomputing, IEEE Computer Society, 1998, pp. 1-27, https://doi.org/10.1109/SC.1998.10004. (Cited on p. 814)

[116] J. H. Wilkinson, Note on the quadratic convergence of the cyclic Jacobi process, Numer. Math., 4 (1962), pp. 296-300, https://doi.org/10.1007/BF01386321. (Cited on p. 844)

[117] J. H. Wilkinson And C. ReInsch, Handbook for Automatic Computation: Volume II: Linear Algebra, Grundlehren Math. Wiss. 186, Springer-Verlag, New York, 1971, https://doi. org/10.1007/978-3-642-86940-2. (Cited on pp. 811, 812, 844)

[118] P. R. Willems And B. LAnG, A framework for the $M R^{3}$ algorithm: Theory and implementation, SIAM J. Sci. Comput., 35 (2013), pp. A740-A766, https://doi.org/10.1137/ 110834020. (Cited on pp. 829, 832)

Copyright (C) by SIAM. Unauthorized reproduction of this article is prohibited. 
[119] P. R. Willems, B. Lang, And C. VÖmel, Computing the bidiagonal SVD using multiple relatively robust representations, SIAM J. Matrix Anal. Appl., 28 (2006), pp. 907-926, https://doi.org/10.1137/050628301. (Cited on p. 855)

[120] B. В. Zhou AND R. P. BRent, A parallel ring ordering algorithm for efficient one-sided Jacobi SVD computations, J. Parallel Distrib. Comput., 42 (1997), pp. 1-10, https://doi. org/10.1006/jpdc.1997.1304. (Cited on p. 847)

Copyright (c) by SIAM. Unauthorized reproduction of this article is prohibited. 